\title{
PRESENTATIONS ON STANDARD GENERATORS FOR CLASSICAL GROUPS
}

\author{
C.R. LEEDHAM-GREEN AND E.A. O'BRIEN
}

\begin{abstract}
For each family of finite classical groups, and their associated simple quotients, we provide an explicit presentation on a specific generating set of size at most 8. Since there exist efficient algorithms to construct this generating set in any copy of the group, our presentations can be used to verify claimed isomorphisms between representations of the classical group. The presentations are available in MAGMA.
\end{abstract}

In memory of Charles Sims who taught us so much

Contents

1. Introduction

1.1. The main result

1.2. Related work

1.3. Our approach

1.4. Comments on length

1.5. Notation

2. Presentations for the symmetric group and related groups

3. A presentation for $\mathrm{SL}(2, q)$

3.1. Generators and notation

3.2. Presentations for $\operatorname{SL}(2, q)$ for $e>1$

3.3. Presentations for $\mathrm{SL}(2, p)$

3.4. Standard generators for $\mathrm{SL}(2, q)$

4. A presentation for $\mathrm{SU}(3, q)$

4.1. Generators and notation

4.2. A presentation for $\mathrm{SU}(3, q)$ for $q>2$

4.3. A presentation for $\mathrm{SU}(3,2)$

4.4. A presentation for $\operatorname{PSU}(3, q)$

4.5. Standard generators for $\mathrm{SU}(3, q)$

5. A presentation for $\operatorname{SL}(d, q)$ for $d>2$

5.1. Generators and notation

5.2. A presentation for $\operatorname{SL}(d, q)$ for $e>1$

5.3. A presentation for $\operatorname{SL}(d, p)$

Date: July 29, 2019.

Both authors were supported by the Marsden Fund of New Zealand via grant UOA 1323. O'Brien thanks the Hausdorff Institute, Bonn, for its hospitality while this work was completed. We thank Peter Brooksbank, Marston Conder, George Havas, Derek Holt, Alexander Hulpke, Bill Kantor, Martin Kassabov, and Martin Liebeck for helpful comment and discussion. 
5.4. A presentation for $\operatorname{PSL}(d, q)$

5.5. Standard generators for $\operatorname{SL}(d, q)$

6. A presentation for $\operatorname{Sp}(2 n, q)$ for $n>1$

6.1. Generators and notation

6.2. A presentation for $\operatorname{Sp}(2 n, q)$ for $e>1$

6.3. A presentation for $\operatorname{Sp}(2 n, p)$

6.4. A presentation for $\operatorname{PSp}(2 n, q)$

6.5. Standard generators for $\operatorname{Sp}(2 n, q)$

7. A presentation for $\mathrm{SU}(2 n, q)$ for $n>1$

7.1. Generators and notation

7.2. A presentation for $\mathrm{SU}(2 n, q)$

7.3. A presentation for $\operatorname{PSU}(2 n, q)$

7.4. Standard generators for $\mathrm{SU}(2 n, q)$

8. A presentation for $\mathrm{SU}(2 n+1, q)$ for $n>1$

8.1. Generators and notation

8.2. A presentation for $\mathrm{SU}(2 n+1, q)$

8.3. A presentation for $\operatorname{PSU}(2 n+1, q)$

19

8.4. Standard generators for $\mathrm{SU}(2 n+1, q)$

9. A presentation for $\Omega^{+}(2 n, q)$ for $n>1$

9.1. Odd characteristic

9.2. Even characteristic

10. A presentation for $\Omega(2 n+1, q)$

10.1. Generators and notation 44

10.2. A presentation for $\Omega(2 n+1, q)$ for $n \geq 2$ and $e>1$

10.3. A presentation for $\Omega(2 n+1, p)$ for $n \geq 2$

10.4. Standard generators for $\Omega(2 n+1, q) \geq 48$

11. A presentation for $\Omega^{-}(2 n, q)$ for $n>1 \quad 48$

11.1. Odd characteristic 48

11.2. Even characteristic 52

12. Verification and access to results 5.5

References 55

\section{INTRODUCTION}

The finite simple groups of Lie type play a fundamental role in many areas of mathematics. Various presentations of these groups satisfying specific requirements are known. Steinberg [28, 29] gave the first presentations. An important variation is that of CurtisSteinberg-Tits (see [12, Theorem 2.9.3]). Motivated in part by a conjecture of [3], Babai et al. [4] provided presentations on a reduced set of Curtis-Steinberg-Tits generators. Guralnick et al. [13, 14 provide short presentations (defined below) for every finite simple group of Lie type except the Ree groups ${ }^{2} G_{2}(q)$. In this paper, we focus on the classical groups. For each family of finite classical groups, and their associated simple quotients, we provide an explicit presentation on a specific generating set of size at most 8 . The most important feature of our work is that there exist efficient algorithms to construct 
this generating set in any copy of the group, so our presentations can be used in practice to verify claimed isomorphisms between representations of the classical group. Encodings of the resulting presentations are available in MAGma [2].

The principal motivation for our work is the matrix group recognition project [25]; it aims to develop high-quality algorithms to compute efficiently with an arbitrary subgroup $G$ of $\mathrm{GL}(m, K)$, where $K$ is a finite field and $G$ is specified by a generating set $Y$. The CompositionTree algorithm [1 constructs a composition series for $G$, and facilitates membership testing in $G$ and writing elements of $G$ as words in $Y$. Additional functionality building on the data structure provided by CompositionTREe includes, for example, the computation of chief series, the soluble radical, and Sylow subgroups. A highly optimised implementation of this machinery is available in MAGMA.

Central to that work is a requirement to produce a concrete isomorphism between an arbitrary representation of a quasisimple group and (a central quotient of) its standard copy, a specific representation of the group. The standard copy of $A_{n}$ is the group acting on $n$ symbols. Our principal focus here is on the classical groups: namely, $\operatorname{SL}(d, q), \operatorname{Sp}(2 n, q)$, $\mathrm{SU}(d, q), \Omega^{+}(2 n, q), \Omega(2 n+1, q)$, and $\Omega^{-}(2 n, q)$, where $d \geq 2, n \geq 1$ and $q$ is a prime power; the standard copy of each is a natural representation of the group preserving a designated classical form. The standard copy of a quasisimple exceptional group of Lie type over $\operatorname{GF}(q)$ is defined in [15]: a specific faithful irreducible representation of minimal dimension of the simply connected group.

Let $C$ be the standard copy of a quasisimple group and let $H=\langle X\rangle \leq \mathrm{GL}(m, K)$ be isomorphic to $C / Z$ where $Z$ is central in $C$. Informally, a constructive recognition algorithm for $H$ constructs an isomorphism between $H$ and $C / Z$ and exploits this isomorphism to write an arbitrary element of $H$ as a word in its generators $X$. For a more formal definition, see [26, p. 192].

We can realise such an algorithm as follows. For each quasisimple group $C$, we define a specific sequence of standard generators $\mathcal{S}$. The first task is to construct, as words in $X$, a sequence $\mathcal{S}^{\prime}$ in $H$ such that $\mathcal{S} \rightarrow \mathcal{S}^{\prime}$ induces an isomorphism from $C / Z$ to $H$. The second task is to solve the constructive membership problem for $H$ with respect to $\mathcal{S}^{\prime}$ : namely, express any given $h \in H$ as a word in $\mathcal{S}^{\prime}$, and so as a word in $X$. Now the isomorphism $\varphi: H \rightarrow C / Z$ that maps $\mathcal{S}^{\prime}$ to the images of $\mathcal{S}$ in $C / Z$ is constructive: any given $h \in H$ may be written as a word $w\left(\mathcal{S}^{\prime}\right)$ in $\mathcal{S}^{\prime}$, and the image $\varphi(h)$ is immediately determined by the corresponding word in the images of $\mathcal{S}$ in $C / Z$. Similarly, the inverse of $\varphi$ is constructive.

Standard generators have been defined and associated constructive recognition algorithms developed for all of the quasisimple groups. The standard generators of $A_{n}$ are a 3 -cycle and an $(n-1)$ - or $n$-cycle depending on the parity of $n$; for a recognition algorithm, see [18]. For exceptional groups of rank at least 2, see [8, 23]. For the sporadic simple groups, see [31, 32]. For the classical groups, see [10, 11, 21]; we use algorithms of Costi [9] and Schneider to solve the constructive membership problem. In all cases, the algorithms demonstrate both excellent theoretical and practical performance. For example, those in [10, 21] have complexity $O\left(r^{4} \log q\right)$ where the classical group has rank $r$. Implementations are available in MAGMA.

All existing constructive recognition algorithms employ random selection in $H$ and are Monte Carlo; consequently, there is a small probability of error in the claimed isomorphism 
between $H=\langle X\rangle \leq \mathrm{GL}(m, K)$ and $C / Z$. Presentations on standard generators for (central quotients of) quasisimple groups play a fundamental role: namely, they permit us to verify the correctness of this isomorphism. Assume we know a presentation $\{\mathcal{S} \mid \mathcal{R}\}$ for $C / Z$. The standard generators $\mathcal{S}^{\prime}$ are constructed as words in $X$, so demonstrating that $\langle X\rangle \geq\left\langle\mathcal{S}^{\prime}\right\rangle$; we use the constructive membership algorithm to write the input generators $X$ as words in $\mathcal{S}^{\prime}$, so demonstrating that $\langle X\rangle \leq\left\langle\mathcal{S}^{\prime}\right\rangle$; finally, if the relators in $\mathcal{R}$ evaluate to the identity on $\mathcal{S}^{\prime}$, then we have verified the claimed isomorphism.

As part of CompositionTree, we must often construct both a homomorphism $\theta$ from $G \leq \mathrm{GL}(m, K)$ onto the simple quotient of a specified classical group $C$, and its associated kernel. We apply (a natural generalisation of) a constructive recognition algorithm to $G$, so constructing $\mathcal{S}^{\prime}$; now evaluating $\mathcal{R}$ on $\mathcal{S}^{\prime}$ provides normal generators for $\operatorname{ker} \theta$. More generally, if we construct a composition tree for an arbitrary group $G=\langle Y\rangle \leq \mathrm{GL}(m, K)$, have a constructive membership test for each of its composition factors, and know a presentation on the standard generators of each factor, then, as we outline in [1, Section 4], we can construct a presentation for $G$ on $Y$; this allows us to verify the correctness of the composition tree constructed for $G$.

Presentations on standard generators for $A_{n}$ are well known. For exceptional groups of rank at least 2, the requisite presentations are listed in [23]; for (most of) the sporadic simple groups, they are listed in the Atlas [32. In this paper, we address the missing case by listing explicit presentations on standard generators for the classical groups and their central quotients.

A more detailed account of the background and context of this work appears in the survey [25].

1.1. The main result. Our motivations dictate the following desiderata for presentations of classical groups.

(1) The presentation is correct.

(2) The presentation can be used to verify the claimed isomorphism.

(3) The number of relators is small.

(4) The presentation length is "short".

The requirement that the presentation is correct may seem obvious, but it is not easy to achieve. We have concentrated on producing presentations whose correctness is easy to verify.

The second is realised by providing a presentation on the standard generators of the group.

The desire for a small number of relators arises because we may have a group $G$ that maps onto a classical group $C$, and a normal subgroup $K$ of $G$ contained in the kernel of the homomorphism of $G$ onto $C$. To verify that $K$ is the full kernel, we must test that a preimage of each relator belongs to $K$.

We seek a "short" presentation to minimise the cost of isomorphism verification. Babai and Szemerédi [3] define the length of a presentation $\{X \mid R\}$ to be the number of symbols required to write down the presentation. Typically, two metrics are applied to measure the length. One is word length: $|X|$ plus the sum of the lengths of the words in $R$ as strings in the alphabet $X \cup X^{-1}$. A second is bit-length: $|X|$ plus the total number of bits required to encode the words in $R$ where all exponents are encoded as binary strings. 
As discussed in [13], we can readily convert a presentation having bit-length $N$ into a presentation having word length $4 N$. As we show in Section 1.4, for our applications the critical metric is bit-length.

We now state the main result, observing that it satisfies these desiderata. Each classical group has at most eight standard generators, as defined in [10, 21].

Theorem 1.1. Every classical group of rank $r$ defined over $\operatorname{GF}(q)$ has a presentation on its standard generators with $O(r)$ relations and total bit-length $O(r+\log q)$.

For each family of classical groups, and their central quotients, we exhibit such a presentation. Sometimes, for the convenience of theoretical arguments, we list a presentation on a (mildly) different generating set. If so, we record explicitly the relationships between those generators and the standard generators needed to rewrite the presentation on the standard generators; see, for example, [19, §4.4, Remark 4].

Our presentation for a classical group $C$ of rank at least 2 on its standard generators usually includes one for the normaliser of a maximal torus in a split $B N$ pair, and so for $S_{n}$, the symmetric group of relevant degree $n$, or the corresponding group of signed permutation matrices of determinant 1 . Our generators for $S_{n}$ in its action on $n$ symbols are a 2 -cycle and $n$-cycle, and every presentation for $S_{n}$ on this generating set has word length $O(n)$ (see [14, A2]). At most 56 additional relations are used to define $C$.

The structure of the paper is the following. In Section 2 we provide presentations for $S_{n}$ and for the group of signed permutation matrices of degree $n$ and determinant 1 . In Sections 3 and 4 we discuss important base cases: $\mathrm{SL}(2, q)$ and $\mathrm{SU}(3, q)$. In subsequent sections, we provide presentations for each of $\mathrm{SL}(d, q), \operatorname{Sp}(2 n, q), \mathrm{SU}(d, q), \Omega^{+}(2 n, q)$, $\Omega(2 n+1, q)$, and $\Omega^{-}(2 n, q)$.

The presentations and corresponding matrix generators are available [22] in MAGMA. As we report in Section 12, we used these extensively to verify the correctness of this work.

1.2. Related work. Babai and Szemerédi [3] formulated the Short Presentation Conjecture: there exists a constant $c$ such that every finite simple group $G$ has a presentation of bit-length $O\left(\log ^{c}|G|\right)$. The results of [4, 16, 30] establish this conjecture with $c=2$ for all finite simple groups, with the possible exception of the Ree groups ${ }^{2} G_{2}(q)$.

The conjecture was motivated by potential complexity applications to questions about matrix groups defined over finite fields (see [3] for details); its proof also provided verification for the first constructive recognition algorithms for classical groups, developed by Kantor and Seress [20.

In two remarkable papers, Guralnick et al. [13, 14] establish much more. We summarise just two of their results.

Theorem 1.2. Every non-abelian finite simple group of rank $r$ defined over $\mathrm{GF}(q)$, with the possible exception of the Ree groups ${ }^{2} G_{2}(q)$, has a presentation with a bounded number of generators and relations and word length $O(\log r+\log q)$. Every finite quasisimple group of rank $r$ defined over $\mathrm{GF}(q)$, with the possible exception of the Ree groups ${ }^{2} G_{2}(q)$, has a presentation with at most 9 generators, 49 relations, and bit-length $O(\log r+\log q)$.

Word and bit-length $O(\log r+\log q)$ are both best possible, and such presentations are short. 
Rarely do we know explicit words (short or otherwise) to express standard generators in terms of the generators used in [14], or vice versa, so it is not feasible to convert their presentations to ones on standard generators. One significant obstruction is that sometimes generators used there are identified only by specifying properties they must satisfy. Nor do constructive recognition algorithms employing their generators exist for classical groups, so these presentations cannot be used directly to verify the necessary isomorphisms. Theorem 10.1 of 14 illustrates an additional concern: it employs a generator $\sigma$ specified only up to certain properties. This element does not exist in $\Omega^{+}(2 n, q)$; in private communication in 2012, the authors of [14] fixed the error. Huxford [17] also corrects various errors in the presentations given there for $A_{n}$ and $S_{n}$. Thus, while the results of [13, 14] provide spectacular answers to long-standing challenging problems, we believe that our presentations are necessary for our significant algorithmic application.

1.3. Our approach. Our general strategy to construct a presentation for a classical group $C$ is straight-forward.

Our generating set usually contains generators for the normaliser $N$ of a maximal torus in a split $B N$ pair, and one root element of each type. For example, we take $\left(\begin{array}{ll}1 & 1 \\ 0 & 1\end{array}\right)$ as our root element in $\operatorname{SL}(d, q)$, and both a short and long root element in $\operatorname{Sp}(2 n, q)$.

We take, as our base cases, groups $H$ of minimum rank, subject to containing root elements of all types. For example, $H=\mathrm{SL}(2, q)$ in the case of $\operatorname{SL}(d, q)$. These groups lie naturally in the larger groups of the same type, and contain our chosen root elements.

Our presentation $\{\mathcal{S} \mid \mathcal{R}\}$ for $C$ always contain four sets of relations: a presentation for $N$; presentations for the subgroups $H$; relations that give the centraliser in $N$ of certain root elements; and certain Steinberg relations. In some cases extra relations are required when $C$ has an exceptional Schur multiplier.

For Chevalley groups, the sufficiency of the Steinberg relations is due to [27, 29]; for the twisted groups it follows from [4]. The Steinberg relations consist of relations that hold in the root subgroups, and relations that express commutators of root elements in terms of other root elements. A sufficient number of relations of this second kind is included in $\mathcal{R}$ by ensuring that one is chosen, or is implied, from each orbit of the action of $N$ on the set of unordered pairs of distinct root elements.

1.4. Comments on length. Formally, we define a presentation of a group $G$ to be of the form $\{X, Y \mid W, R\}$, where $X$ is the given generating set, $Y=\left\{y_{1}, \ldots, y_{n}\right\}$ are auxiliary generators, $W=\left\{w_{1}, \ldots, w_{n}\right\}$ are words where $w_{i}$ is a word on $X \cup\left\{w_{1}, \ldots, w_{i-1}\right\}$ which defines $y_{i}$, and $R$ is a defining set of relators for $G$, expressed as words in $X \cup Y$, and hence, with the use of $W$, as words in $X$. The sets $Y$ and $W$ are simply tools for expressing the relators $R$ efficiently as words in $X$, and should not be counted either as generators or relations. For example, the generators $a=\Delta^{x}$ and $b=\Delta^{y}$ in the presentation for $\mathrm{SU}(3, q)$ given in Section 4 are superfluous, so we do not count $a$ and $b$ as generators, nor their definitions as relations. Variations of this approach may be used to convert a presentation of bit-length $N$ into one of word length $4 N$. We also store words as straight-line programs on $X \cup Y$; for a formal definition and discussion of their significance, see [26, p. 10]. The most important use of straight-line programs rather than words is when we raise a word to a high power. There are fast algorithms for raising a matrix over a finite field to a high power (see [21, Section 10]); so our straight-line programs encode a cell as a power of an 
earlier cell. Hence, for our purposes, the critical metric to assess presentation length is bit-length.

1.5. Notation. The dihedral group of order $2 n$, where $n$ is positive, is

$$
D_{2 n}:=\left\langle a, b \mid b^{n}=1, b^{a}=b^{-1}, a^{2}=1\right\rangle .
$$

The generalised quaternion group of order $2 n$, where $n$ is positive and even, is

$$
Q_{2 n}:=\left\langle a, b \mid b^{n}=1, b^{a}=b^{-1}, a^{2}=b^{n / 2}\right\rangle .
$$

The semidihedral group of order $2 n$, where $n$ is positive and $n \equiv 0 \bmod 4$, is

$$
S D_{2 n}:=\left\langle a, b \mid b^{n}=1, b^{a}=b^{-1+n / 2}, a^{2}=1\right\rangle .
$$

Note that we generalise the usual definitions of these groups.

\section{Presentations for the symmetric group and Related Groups}

Let $d$ be a positive integer. Define $\epsilon_{d}=(-1)^{d+1}$. If $\epsilon= \pm 1$, and $\left(a_{1}, a_{2}, \ldots, a_{d}\right)$ is a cyclic permutation, then we define the signed permutation $\left(a_{1}, a_{2}, \ldots, a_{d}\right)^{\epsilon}$ by $a_{i} \mapsto a_{i+1}$ for $i<d$, and $a_{d} \mapsto \epsilon a_{1}$. In particular, $(a)^{-}$is the transposition that interchanges $a$ and $-a$.

For the symmetric group on $\{1,2, \ldots, d\}$, our generating set is $\{U, V\}$, where $U$ and $V$ stand for the permutations $(1,2)$ and $(1,2, \ldots, d)$ respectively.

Let $U^{\prime}=(1,2)^{-}$and $V^{\prime}=(1,2, \ldots, d)^{\epsilon_{d}}$ be permutations on $\{ \pm 1, \pm 2, \ldots, \pm d\}$; so $\left\langle U^{\prime}, V^{\prime}\right\rangle$ may be taken as the group of signed permutation matrices of determinant 1 , a subgroup of index 2 in the group of all signed permutations of degree $d$.

Moore [24, p. 358] gave the following presentation for $S_{d}$.

Theorem 2.1. The symmetric group $S_{d}$, for $d>2$, has the following presentation:

$$
\left\{U, V \mid U^{2}=V^{d}=(U V)^{d-1}=\left(U U^{V}\right)^{3}=\left(U U^{V^{j}}\right)^{2}=1 \text { for } 2 \leq j \leq d / 2\right\} .
$$

This presentation for $S_{d}$ on its standard generators has word length $O\left(d^{2}\right)$ and bitlength $O(d \log d)$, and can easily be rewritten to have word length $O(d)$ by introducing auxiliary generators $V_{j}=V^{j}$, with relations $V_{j}=V V_{j-1}$.

At the cost of introducing relations with bit-length $O(d \log d)$, we could convert the presentation for $S_{d}$ in [5, Theorem 1.2] of bit-length $O(\log d)$ to one on generators $U$ and $V$ that has an absolutely bounded number of relations.

We now give presentations for the group of signed permutation matrices of determinant 1 and degree $d$. By introducing auxiliary generators, these also achieve word length $O(d)$.

Theorem 2.2. The group of signed permutation matrices of determinant 1 and degree $d$, when $d>2$ is odd, has the following presentation:

$$
\begin{gathered}
\left\{U^{\prime}, V^{\prime} \mid U^{\prime 4}=U^{\prime 2 V^{\prime} U^{\prime}} U^{\prime 2} U^{\prime 2 V^{\prime}}=V^{\prime d}=\left(U^{\prime} V^{\prime}\right)^{d-1}=\left(U^{\prime} U^{\prime V^{\prime}}\right)^{3}=\right. \\
\left.\left[U^{\prime}, U^{\prime V^{\prime j}}\right]=1 \text { for } 2 \leq j \leq(d-1) / 2\right\} .
\end{gathered}
$$


Proof. These relations are readily verified. The relations $\left[U^{\prime}, U^{\prime V^{\prime j}}\right]=1$ for $2 \leq j \leq$ $(d-1) / 2$ extend, by conjugation with $V^{\prime d-j}$, to allow $2 \leq j \leq d-2$. Let $G$ denote the presented group, and $A$ the normal closure in $G$ of $a:=U^{\prime 2}$. By the previous theorem, $G / A$ is isomorphic to $S_{d}$, the relation $\left[U, U^{V^{j}}\right]=1$ being equivalent to the relation $\left(U U^{V^{j}}\right)^{2}=1$ in the presence of the relation $U^{2}=1$.

We prove that $B:=\left\langle\left\{a_{j+1}:=a^{V^{\prime j}} \mid 0 \leq j<d\right\}\right\rangle$ is an elementary abelian 2-group. This generating set is permuted cyclically by $V^{\prime}$. The relation $U^{\prime 2 V^{\prime} U^{\prime}} U^{\prime 2} U^{2 V^{\prime}}=1$ states that $a_{2}^{U^{\prime}} a_{1} a_{2}=1$, so $a_{1} a_{2}$ is an involution, and hence $a_{1}$ commutes with $a_{2}$. It follows, by conjugation with $V^{\prime-1}$, that $a_{d}$ commutes with $a_{1}$. But $a_{1}$ commutes with $a_{j}$ for $3 \leq j \leq d-1$ since $U^{\prime}$ commutes with $U^{\prime V^{j}}$ for $2 \leq j \leq d-2$. So $a_{1}$ commutes with $a_{j}$ for all $j$, and conjugating by $V^{\prime i}$ then proves that $a_{i}$ and $a_{j}$ commute for all $i$ and $j$.

We now prove that $a_{1} a_{2} \ldots a_{d}=1$. Clearly $U^{\prime}$ commutes with $a_{j+1}=\left(U^{\prime V^{\prime j}}\right)^{2}$ for $2 \leq j \leq d-1$, so

$$
a_{1}^{\left(U^{\prime} V^{\prime}\right)^{d-1}}=a_{2}^{\left(U^{\prime} V^{\prime}\right)^{d-2}}=\left(a_{2} a_{3}\right)^{\left(U^{\prime} V^{\prime}\right)^{d-3}}=\left(a_{2} a_{3} a_{4}\right)^{\left(U^{\prime} V^{\prime}\right)^{d-4}}=\cdots=a_{2} a_{3} \ldots a_{d} .
$$

But $\left(U^{\prime} V^{\prime}\right)^{d-1}=1$, so $a_{1} a_{2} \ldots a_{d}=1$, as required. Hence $a_{d}^{U^{\prime}}=a_{1} a_{d}$; so $B$ is a normal subgroup of $G$, and the result follows.

The following is proved similarly.

Theorem 2.3. The group of signed permutation matrices of determinant 1 and degree $d$, when $d>2$ is even, has the following presentation:

$$
\begin{gathered}
\left\{U^{\prime}, V^{\prime} \mid U^{\prime 4}=U^{\prime 2 V^{\prime} U^{\prime}} U^{\prime 2} U^{\prime 2 V^{\prime}}=\left(U^{\prime} U^{\prime V^{\prime}}\right)^{3}=\left[U^{\prime}, U^{\prime V^{\prime j}}\right]=1 \text { for } 2 \leq j \leq d / 2,\right. \\
\left.V^{\prime d}=\left(U^{\prime} V^{\prime}\right)^{d-1},\left[V^{\prime d}, U^{\prime}\right]=1, V^{\prime 2 d}=1\right\} .
\end{gathered}
$$

If $d=2$, then the group is cyclic of order 4 .

We shall frequently need the following result.

Theorem 2.4. Let $d>3$. The group $H$ generated by $U^{\prime V^{\prime 2}}$ and $V^{\prime} U^{\prime-1} U^{\prime-V^{\prime}}$ is the group of signed permutation matrices of $1, \ldots, d$ having determinant 1 that fixes both 1 and 2 . The group $G$ generated by $U^{\prime V^{\prime 2}}$ and $V^{\prime} U^{\prime} U^{\prime V^{\prime}}$ is the direct product of $H$ with the cyclic group of order 2 generated by $(1)^{-}(2)^{-}$.

Proof. Observe that $U^{\prime V^{\prime 2}}=(3,4)^{-}$and $V^{\prime} U^{\prime-1} U^{\prime-V^{\prime}}=(3,4, \ldots, d)^{\epsilon_{d}}$, which proves the first statement. Since $V^{\prime} U^{\prime} U^{\prime V^{\prime}}=(1)^{-}(2)^{-}(3,4, \ldots, d)^{\epsilon_{d}}$ it follows that $G$ maps onto $H$, with kernel $\left\langle(1)^{-}(2)^{-}\right\rangle$. The subgroup of $G$ generated by $(3,4)^{-}$and its conjugates is isomorphic to $H$, so the extension splits.

\section{A presentation for $\operatorname{SL}(2, q)$}

3.1. Generators and notation. Let $q=p^{e}$ for a prime $p$. Let $\omega$ be a primitive element of $\operatorname{GF}(q)$. We define the following elements of $\operatorname{SL}(2, q)$.

$$
\begin{aligned}
& \tau(\alpha)=\left(\begin{array}{cc}
1 & \alpha \\
0 & 1
\end{array}\right) \text { for } \alpha \in \operatorname{GF}(q) ; \\
& \delta=\left(\begin{array}{cc}
\omega^{-1} & 0 \\
0 & \omega
\end{array}\right) ; \\
& U=\left(\begin{array}{cc}
0 & 1 \\
-1 & 0
\end{array}\right) .
\end{aligned}
$$


3.2. Presentations for $\operatorname{SL}(2, q)$ for $e>1$. We use presentations from [7] rewritten on the generating set $\{\tau=\tau(1), \delta, U\}$. A complication arises from the fact that $\tau(\alpha)^{\delta}=\tau\left(\alpha \omega^{2}\right)$, so $\tau\left(\omega^{i}\right)$ is treated differently according to the parity of $i$. In the presented groups given below, $\tau$ is a generator, and $\tau_{1}$, standing for $\tau(\omega)$, is defined as a product of conjugates of $\tau$ by powers of $\delta$. Now $\tau_{i}$ may be defined for $0 \leq i \leq e$ by $\tau_{0}=\tau$ and $\tau_{i}=\tau_{i-2}^{\delta}$ if $i>1$, so $\tau_{i}$ stands for $\tau\left(\omega^{i}\right)$. If $f(t)=\sum_{i=0}^{e} u_{i} t^{i}$ is a polynomial over $\operatorname{GF}(p)$ of degree $e$, then we write $\tau^{f(t)}$ for $\prod_{i} \tau_{i}^{u_{i}}$.

Theorem 3.1. Let $q=p^{e}$ for an odd prime $p$ and $e>1$. Let $f(t)$ be the minimal polynomial of $\omega$ over $\operatorname{GF}(p)$. Write $\omega=\sum_{i=0}^{e-1} a_{i} \omega^{2 i}$. Let $m$ satisfy the equation $\omega^{2 m}=$ $1+\omega$ if $1+\omega \in \operatorname{GF}(q)^{2}$, and $\omega^{2 m+1}=1+\omega$ otherwise. Then $\operatorname{PSL}(2, q)$ has the following presentation:

$$
\begin{gathered}
\left\{\tau, \delta, U \quad \mid \tau_{1}:=\prod_{i} \tau^{a_{i} \delta^{i}},(\tau U)^{3}=(U \delta)^{2}=U^{2}=\left(\tau_{1} U \delta\right)^{3}=\delta^{(q-1) / 2}=\tau^{p}=1,\right. \\
{\left[\tau, \tau_{1}\right]=\left[\tau_{1}, \tau^{\delta}\right]=\tau^{f(t)}=1 ;} \\
\text { if } \left.1+\omega \in \mathrm{GF}(q)^{2}, \text { then } \tau^{\delta^{m}}=\tau \tau_{1}, \tau_{1}^{\delta^{m}}=\tau_{1} \tau^{\delta} \text {; otherwise } \tau_{1}^{\delta^{m}}=\tau \tau_{1}, \tau^{\delta^{m+1}}=\tau_{1} \tau^{\delta}\right\} .
\end{gathered}
$$

This is essentially due to Campbell, Robertson, and Williams [7, Theorem 2.2]. We have replaced their generators $w, x, y, z$ by $U \tau^{-1}, \tau, \tau_{1}$, and $\tau \delta^{-1}$. The proof that the elements $\tau_{i}$ in the presented group $G$ commute goes through unchanged with our presentation. They have relations equivalent to $\tau^{p}=1$ and $\tau_{1}^{p}=1$. The second of these is redundant, as $\tau_{1}$ is a product of commuting conjugates of $\tau$. The relation $\tau^{f(t)}=1$ gives the action of $\delta$ on the irreducible module generated by the $\tau_{i}$; their equivalent relation with $\tau$ replaced by $\tau_{1}$ is also redundant. Their relation $z^{(q-1) / 2}=1$ translates to $\left(\tau \delta^{-1}\right)^{(q-1) / 2}=1$ in our notation, and we have replaced it by the relation $\delta^{(q-1) / 2}=1$. Given the action of $\delta$ on the module generated by the $\tau_{i}$, these relations are equivalent. Finally we have replaced their relation $(w y z)^{3}=1$, which translates to $\left(U \tau^{-1} \tau_{1} \tau \delta^{-1}\right)^{3}=1$, by the relation $\left(\tau_{1} U \delta\right)^{3}=1$. These are equivalent, since both $\left[\tau, \tau_{1}\right]=1$ and $U \delta=\delta^{-1} U$ hold in $G$.

Theorem 3.2. A presentation for $\operatorname{SL}(2, q)$, where $q=p^{e}$ is odd and $e>1$, is obtained from the presentation for $\operatorname{PSL}(2, q)$ in Theorem 3.1 by replacing the relations

$$
(\tau U)^{3}=1, \quad(U \delta)^{2}=1, \quad U^{2}=1, \quad \delta^{(q-1) / 2}=1
$$

by the relations

$$
\left(\tau U^{-1}\right)^{3}=U^{2}, \quad(U \delta)^{2}=U^{2}, \quad U^{4}=1, \quad \delta^{(q-1) / 2}=U^{2} .
$$

Proof. The given relations all hold in $\operatorname{SL}(2, q)$. It remains to prove that $U^{2}$ is central in the presented group. The relations $\left(\tau U^{-1}\right)^{3}=U^{2}$ and $(U \delta)^{2}=U^{2}$ imply that $\tau$ and $\delta$ commute with $U^{2}$, and the result follows.

These presentations may be significantly simplified if $q \equiv 3 \bmod 4$.

Theorem 3.3. With the notation of Theorem [3.1, if $q \equiv 3 \bmod 4$ and $e>1$, then $\operatorname{PSL}(2, q)$ has the following presentation:

$$
\left\{\tau, \delta, U \mid(\tau U)^{3}=(U \delta)^{2}=U^{2}=\left[\tau, \tau^{\delta^{(q+1) / 4}}\right]=\tau^{f(t)}=1, \delta^{(q-1) / 2}=\tau^{p}, \tau^{\delta^{m}}=\left[\tau^{-1}, \delta^{r}\right]\right\},
$$

where $r=(q+1) / 4$ if $1+\omega \in \mathrm{GF}(q)^{2}$, and $r=(q-3) / 4$ otherwise. 
This is essentially [7, Theorem 2.4]. We have replaced the relation that translates to $\left(\tau \delta^{-1}\right)^{(q-1) / 2}=1$ by the simpler relation $\delta^{(q-1) / 2}=1$, as in Theorem 3.1. The relation $\tau^{p}=1$ may be retrieved as follows. We have the relations $\delta^{(q-1) / 2}=\tau^{p}$ and $\tau^{\delta^{m}} \tau^{\delta^{r}}=\tau$, where the latter is a rewriting of $\tau^{\delta^{m}}=\left[\tau^{-1}, \delta^{r}\right]$. Since $\tau^{p}$ commutes with $\delta$ it follows that $\tau^{p} \tau^{p}=\tau^{p}$.

Note that $\tau^{\delta^{(q+1) / 4}}$ is $\tau(-\omega)$, and plays the role of $\tau_{1}=\tau(\omega)$ in Theorem 3.1. It is because we can write this element as a conjugate of $\tau$ that we have a simpler presentation when $q \equiv 3 \bmod 4$.

Theorem 3.4. If $q \equiv 3 \bmod 4$ and $e>1$, then a presentation for $\operatorname{SL}(2, q)$ is obtained from the presentation for $\operatorname{PSL}(2, q)$ in Theorem 3.3 by replacing the relations

$$
(\tau U)^{3}=1, \quad(U \delta)^{2}=1, \quad U^{2}=1, \quad \delta^{(q-1) / 2}=\tau^{p}
$$

by the relations

$$
\left(\tau U^{-1}\right)^{3}=U^{2}, \quad(U \delta)^{2}=U^{2}, \quad U^{4}=1, \quad \delta^{(q-1) / 2}=\tau^{p} U^{2} .
$$

The proof is similar to that of Theorem 3.2 .

Similar results hold for characteristic 2. Translated into our generating system, [7, Theorem 3.2] states the following.

Theorem 3.5. If $e>1$, and $m$ satisfies the equation $\omega^{2 m}=1+\omega^{2}$, and the minimum polynomial of $\omega^{2}$ over $\mathrm{GF}(2)$ is $\sum_{i} u_{i} t^{i}$, then $\mathrm{SL}\left(2,2^{e}\right)$ has the following presentation:

$$
\left\{\tau, \delta, U \mid(U \tau)^{3}=U^{2}=(U \delta)^{2}=(\tau \delta)^{q-1}=\tau^{2}=1, \tau^{\delta^{m}}=[\tau, \delta], \prod_{i} \tau^{u_{i} \delta^{i}}=1\right\} .
$$

3.3. Presentations for $\mathrm{SL}(2, p)$. We use the following presentations from [6] rewritten on the generating set $\{\tau, U\}$.

Theorem 3.6. Let $p$ be an odd prime and let $k=\lfloor p / 3\rfloor$. If $p \equiv 1 \bmod 3$ then $\operatorname{SL}(2, p)$ has presentation

$$
\left\{\tau, U \mid U^{2}=\left(U \tau U^{2}\right)^{3},\left(U\left(\tau U^{2}\right)^{4} U\left(\tau U^{2}\right)^{(p+1) / 2}\right)^{2}\left(\tau U^{2}\right)^{p} U^{2 k}=1\right\}
$$

else

$$
\left\{\tau, U \mid U^{-2}=\left(U^{-1} \tau\right)^{3},\left(U^{-1} \tau^{4} U^{-1} \tau^{(p+1) / 2}\right)^{2} \tau^{p} U^{-2 k}=1\right\} .
$$

Note that $\operatorname{SL}(2,2)$ has presentation $\left\{\tau, U \mid(\tau U)^{3}=U^{2}=\tau^{2}=1\right\}$. If $e=1$ then we may take $\omega$ as an integer. Observe that $\delta=\left(\tau^{\omega-\omega^{2}}\right) U \tau^{\omega^{-1}}\left(\tau^{\omega-1}\right)^{U} \tau^{-1}$; in particular, $\delta$ is the identity and $U^{2}$ for $p=2,3$ respectively.

A presentation for $\operatorname{PSL}(2, p)$ is obtained by imposing the additional relation $U^{2}=1$.

3.4. Standard generators for $\operatorname{SL}(2, q)$. In Table 1 of [10, 21 the non-trivial standard generators for $\operatorname{SL}(2, q)$ are labelled $s, t, \delta$. Observe that $s=U ; t=\tau$; and the standard generator $\delta$ is the inverse of the presentation generator $\delta$. 


\section{A presentation for $\mathrm{SU}(3, q)$}

4.1. Generators and notation. Let $q=p^{e}$ for a prime $p$. Let $\omega$ be a primitive element of $\operatorname{GF}\left(q^{2}\right)$, and let $\omega_{0}=\omega^{q+1}$, so $\omega_{0}$ is a primitive element of $\operatorname{GF}(q)$.

Let $\xi=1 /\left(1+\omega^{q-1}\right)$ if $q$ is even, and $\xi=-1 / 2$ if $q$ is odd, and let $\zeta=-\omega^{\left(q^{2}+q\right) / 2}$. So $\xi$ has trace -1 , and $\zeta^{2}=\omega_{0}$, and $\zeta$ has trace 0 . Note that $\zeta=\omega^{(q+1) / 2}$ if $q$ is odd.

We work with respect to the basis $\left(e_{1}, v, f_{1}\right)$ for the underlying vector space, where the hermitian form is defined by $e_{1} \cdot f_{1}=f_{1} \cdot e_{1}=v \cdot v=1$, and the form vanishes on all other pairs of basis vectors. Let $K$ be the root group of order $q^{3}$ consisting of all matrices of the form

$$
\nu(\alpha, \beta)=\left(\begin{array}{ccc}
1 & \alpha & \beta \\
0 & 1 & -\alpha^{q} \\
0 & 0 & 1
\end{array}\right),
$$

where $\alpha$ and $\beta$ lie in $\operatorname{GF}\left(q^{2}\right)$, and $\beta$ has trace $-\alpha^{q+1}$, for example, $\beta=\xi \alpha^{q+1}$.

Let $\nu=\nu(1, \xi)$, and let $\tau=\nu(0, \zeta)$ if $q$ is odd, and $\tau=\nu(0,1)$ if $q$ is even. Define

$$
\Delta(\alpha)=\left(\begin{array}{ccc}
\alpha & 0 & 0 \\
0 & \alpha^{q-1} & 0 \\
0 & 0 & \alpha^{-q}
\end{array}\right),
$$

and let $\Delta=\Delta(\omega)$.

Finally let $t=\left(\begin{array}{ccc}0 & 0 & 1 \\ 0 & -1 & 0 \\ 1 & 0 & 0\end{array}\right)$.

If $q>2$ then $\{\nu, \tau, \Delta, t\}$ generates $\mathrm{SU}(3, q)$, but it generates only a subgroup of index 2 in $\mathrm{SU}(3,2)$.

4.2. A presentation for $\mathrm{SU}(3, q)$ for $q>2$. Using the work of [16], Guralnick et al. [14, Theorem 4.10] give a short presentation for $\mathrm{SU}(3, q)$ for $q>2$ on $\left\{\nu, \Delta^{-q}, t\right\}$.

Here, for completeness, we provide a variation of their presentation on generators $\{\nu, \tau, \Delta, t\}$. Let $H=\langle\nu, \tau, \Delta\rangle$, a Borel subgroup of order $\left(q^{2}-1\right) q^{3}$. If $q$ is even, then relation $R_{1}$ (iii) given below defines $\tau$, otherwise our presentation is independent of the choice of a non-trivial element of $\langle\tau\rangle^{H}$; so this difference between the presentations is insignificant. Following the existing approach, we first construct a presentation for $H$, and then identify explicitly the additional relations implied by [16] to obtain one for $\mathrm{SU}(3, q)$.

\subsubsection{A presentation for $H$.}

Lemma 4.1. If $q \neq 2,3,5$ then there exist integers $x$ and $y$ such that

(i) $\omega^{x(q-2)}+\omega^{y(q-2)}=1$;

(ii) $\omega^{-x(q+1)}+\omega^{-y(q+1)}=1$;

(iii) $\mathrm{GF}(p)\left[\omega^{x(q+1)}\right]=\mathrm{GF}(q)$;

(iv) $\mathrm{GF}(p)\left[\omega^{x(q-2)}\right]$ is equal to $\mathrm{GF}\left(q^{2}\right)$ or $\mathrm{GF}(q)$, according as $p$ is odd or even.

Proof. This is [13, Lemma 4.23], with $a$ and $b$ replaced by $\omega^{-x}$ and $\omega^{-y}$ respectively, as can be seen by raising (i) to the power $q$. 
The proof of this lemma in [13] allows us to obtain $x$ and $y$ readily. If $q$ is even, then $\omega^{-x}=\omega_{0}$ and $\omega^{-y}=1-\omega_{0}$. Hence $x=-(q+1)$, and $y=-c(q+1)$ where we solve the equation $\omega_{0}^{c}=1-\omega_{0}$ for $c$ via a discrete $\log$ computation in $\operatorname{GF}(q)$. Otherwise, define $\psi:=\omega^{(q+1) / 2}$ and $c_{t}:=t\left(t^{2}+3 \psi^{2}\right)(t+\psi)\left(t^{2}-\psi^{2}\right)^{-2}$ for $t \in \operatorname{GF}\left(q^{2}\right)$. If $x$ and $y$ satisfy conditions (i) and (ii), then $\omega^{-x}=c_{t}$ and $\omega^{-y}=c_{t}-c_{t}^{q-1}$ for some $t \in \operatorname{GF}\left(q^{2}\right)$. The number of such $t$ in $\operatorname{GF}\left(q^{2}\right)$ is always positive and is at least $q-5-6(e-1) q^{1 / 2}$. Hence we determine $t$ by a random search, and so $x$ and $y$ via a discrete $\log$ computation in $\operatorname{GF}\left(q^{2}\right)$.

We repeatedly use the following which is [13, Lemma 4.5].

Lemma 4.2. Let $U_{0}$ and $W_{0}$ be subgroups of a group $G$, and let $u, w, a, b$ be elements of $G$ satisfying the following conditions:

(i) $[a, b]=1$;

(ii) $\left\langle u, u^{a}, U_{0}\right\rangle=\left\langle u, u^{b}, U_{0}\right\rangle=\left\langle u^{a}, u^{b}, U_{0}\right\rangle$;

(iii) $\left\langle w, w^{a}, W_{0}\right\rangle=\left\langle w, w^{b}, W_{0}\right\rangle=\left\langle w^{a}, w^{b}, W_{0}\right\rangle$;

(iv) $\left[u^{a}, w\right]=\left[u^{b}, w\right]=1$;

(v) $U_{0}$ and $W_{0}$ are normalised by $\langle a, b\rangle$;

(vi) $\left[U_{0}, w\right]=\left[u, W_{0}\right]=1$.

Then $\left[\left\langle\left\{u^{c}: c \in\langle a, b\rangle\right\}\right\rangle,\left\langle\left\{w^{c}: c \in\langle a, b\rangle\right\}\right\rangle\right]=1$.

We build up our presentation for $H$ on the generating set $\{\nu, \tau, \Delta\}$ in steps. Let $G$ be the presented group, so that $G$ changes as relations are imposed.

Let $a=\Delta^{x}$ and $b=\Delta^{y}$, with $x$ and $y$ as in Lemma 4.1. When $e>1$, we choose $x$ such that $\operatorname{gcd}\left(x, q^{2}-1\right)=1$ whenever possible. This guarantees conditions (iii) and (iv) of the lemma.

Let $U$ be the normal closure in $H$ of $\langle\nu, \tau\rangle$, so $U$ is the group of upper unitriangular matrices in $H$. Let $W$ be the normal closure of $\langle\tau\rangle$ in $H$, so $W$ is the centre of $U$.

We use three sets of relations to present $H$. The first $R_{1}$ is the following.

(i) $\Delta^{q^{2}-1}=1$;

(ii) $a=\Delta^{x}$ and $b=\Delta^{y}$;

(iii) $\nu^{p}=1$ for odd $p$, and $\nu^{2}=\tau$ for even $p$;

(iv) $\tau^{p}=1$.

Suppose that $m(s)=\sum_{i} a_{i} s^{i}$ is a polynomial over $\operatorname{GF}(p)$. If $w \in W$ and $h \in\langle\Delta\rangle$, we define $w^{m(h)}$ to be $\prod_{i}\left(w^{a_{i}}\right)^{h^{i}}$, and if $u \in U$ we define $u^{m(h)}$ similarly, but here the order in which the terms are multiplied is relevant. Any fixed order, such as the natural order, may be used.

We now introduce a set $R_{2}$ of relations which, as we shall see, determines, with $R_{1}$, the structure of $W=\left\langle\left\{\tau^{\delta}: \delta \in\langle\Delta\rangle\right\}\right\rangle$.

(i) $\tau=\tau^{a} \tau^{b}$, and, if $p$ is odd, then $\tau=\tau^{b} \tau^{a}$;

(ii) if $e>1$ then $\tau^{m_{1}(a)}=1$, where $m_{1}$ is the minimum polynomial of $\omega_{0}^{-x}$ over $\operatorname{GF}(p)$;

(iii) if $e=1$, or $\operatorname{gcd}\left(x, q^{2}-1\right)>1$, then $\tau^{\Delta}=\tau^{m_{2}(a)}$, where $m_{2}$ is a polynomial of degree at most $e-1$ over $\operatorname{GF}(p)$, and $\omega_{0}^{-1}=m_{2}\left(\omega_{0}^{-x}\right)$.

Relation (i) is satisfied in $H$, since $\omega^{-x(q+1)}+\omega^{-y(q+1)}=1$, Note that $\tau=\tau^{b} \tau^{a}$ also holds in $G$ in the case of even $p$, by $R_{1}(\mathrm{iv})$ and $R_{2}(\mathrm{i})$. Observe that $\left[\tau, \tau^{a}\right]=\left[\tau, \tau^{b}\right]=1$, since $\tau=\tau^{a} \tau^{b}$ and $\left[\tau^{a}, \tau^{b}\right]=1$. 
To see that $R_{1}(\mathrm{ii}), R_{2}(\mathrm{i})$, and this last observation imply that $X:=\left\langle\left\{\tau^{c}: c \in\langle a, b\rangle\right\}\right\rangle$ is abelian, apply Lemma 4.2, with $U_{0}=W_{0}=\langle 1\rangle$, and $u=w=\tau$. Since $a$ and $b$ commute, $R_{2}(\mathrm{i})$ implies that $X=\left\langle\left\{\tau^{c}: c \in\langle a\rangle\right\}\right\rangle$. If $e=1$ then (iii) asserts that $\Delta$ normalises $\langle\tau\rangle$, so this group is equal to $W$, as required. If $e>1$ then (ii) implies that $X$ has order $p^{e}$. If also $\operatorname{gcd}\left(x, q^{2}-1\right)=1$ then $\Delta \in\langle a\rangle$, so $\Delta$ normalises $X$, which is hence equal to $W$, as required. But if $\operatorname{gcd}\left(x, q^{2}-1\right)>1$ then (iii) implies that $X$ is normalised by $\Delta$, as required.

We now introduce a set $R_{3}$ of relations that imply, with $R_{1}$ and $R_{2}$, that $U / W$ is elementary abelian of order $q^{2}$.

(i) $\nu=\nu^{a} \nu^{b} w_{1}$ for some $w_{1} \in W$, and if $p$ is odd then $\nu=\nu^{b} \nu^{a} w_{2}$ for some $w_{2} \in W$;

(ii) $\left[\nu^{a}, \tau\right]=\left[\nu^{b}, \tau\right]=1$;

(iii) $\left[\nu, \nu^{a}\right]=w_{3}$ for some $w_{3} \in W$;

(iv) if $p$ is even, so $\operatorname{GF}(p)\left[\omega^{x(q-2)}\right]=\operatorname{GF}(q)$, and $e>1$, then $\left[\nu^{\Delta}, \nu^{a}\right]=w_{4}$ and $\left[\nu^{\Delta}, \nu^{b}\right]=w_{5}$ for some $w_{4}$ and $w_{5} \in W$

(v) $\nu^{m_{3}(a)}=w_{6}$ for some $w_{6} \in W$, where $m_{3}$ is the minimum polynomial of $\omega^{x(q-2)}$ over $\mathrm{GF}(p)$;

(vi) if $p$ is odd and $\operatorname{gcd}\left(x, q^{2}-1\right)>1$ then $\nu^{\Delta}=\nu^{m_{4}(a)} w_{6}$ for some $w_{6} \in W$, where $m_{4}$ is the polynomial of degree at most $2 e-1$ over $\operatorname{GF}(p)$ satisfying $\omega^{q-2}=m_{4}\left(\omega^{x(q-2)}\right)$; otherwise, if $p$ is even, then $\nu^{\Delta^{2}}=\nu^{m_{5}(a)} \nu^{\Delta m_{6}(a)} w_{6}$ for some $w_{6} \in W$, where $m_{5}$ and $m_{6}$ are the polynomials of degree at most $e-1$ over $\operatorname{GF}(p)$ satisfying $\omega^{2 q-4}=m_{5}\left(\omega^{x(q-2)}\right)+\omega^{q-2} m_{6}\left(\omega^{x(q-2)}\right)$.

Observe that (i) is satisfied in the matrix group since $\omega^{x(q-2)}+\omega^{y(q-2)}=1$. The elements $w_{i}$ need to be determined by calculation in the matrix group.

We first show that these relations imply that $U / W_{1}$ is elementary abelian, of order $q^{2}$, where $W_{1}=\left\langle\left\{\tau^{g}: g \in G\right\}\right\rangle$. Let $Y=\left\langle\left\{\langle\nu, \tau\rangle^{c}: c \in\langle a, b\rangle\right\}\right\rangle\left\langle G\right.$, and $Z=Y W_{1} / W_{1}$. Suppose first that $p$ is odd. The fact that $Z$ is abelian follows from Lemma 4.2 with $u=w=\nu$, and $U_{0}=W_{0}=W_{1}$. Since $a$ and $b$ commute, it follows from (i) that $Z=\left\langle\left\{\nu^{c}: c \in\langle a\rangle\right\}\right\rangle W_{1} / W_{1}$. The fact that $Z$ is elementary abelian follows from $R_{1}$ (iii). It now follows from (v) that $Z$ has order at most $q^{2}$, and hence has order exactly $q^{2}$. Since $\Delta$ commutes with $a$, it follows from (vi) that $Y$ is normalised by $\Delta$. So $Y=U$, and $U / W_{1}$ has order $q^{2}$, as required. If $p$ is even, then we need to prove that $Z$ has order $q$, and that $U / W_{1}$ is the direct sum of $Z$ and $Z^{\Delta}$. The proof that $Z$ is elementary abelian goes through as in the case when $p$ is odd. The fact that $Z$ has order $q$ follows from (v). The fact that $Z$ and $Z^{\Delta}$ commute follows from Lemma 4.2 with $u=\nu$, and $w=\nu^{\Delta}$, and $U_{0}=W_{0}=W_{1}$. The fact that the direct sum of these two groups is normalised by $\Delta$ follows from (vi). It remains to prove that $W_{1}=W$; in other words, that $W$ is normalised by $U$. Since $W$ is normalised by $\Delta$, it suffices to prove that it is normalised by $\nu$, or, equivalently, by $\nu^{a}$. We have seen that $W=\left\langle\left\{\tau^{c}: c \in\langle a, b\rangle\right\}\right\rangle$. But $\left[\left\langle\left\{\tau^{c}: c \in\langle a, b\rangle\right\}\right\rangle,\left\langle\left\{\nu^{c}: c \in\langle a, b\rangle\right\}\right\rangle\right]=1$ by Lemma 4.2, with $u=\tau$, and $w=\nu$, and $U_{0}=\langle 1\rangle$, and $W_{0}=W$.

It follows that $G$ has order at most $q^{3}\left(q^{2}-1\right)$, and hence has precisely this order. Our analysis implies the following.

Theorem 4.3. If $H=\left\langle\Delta, \nu, \tau \mid R_{1} \cup R_{2} \cup R_{3}\right\rangle$, then $H$ is isomorphic to a Borel subgroup of $\mathrm{SU}(3, q)$. 
4.2.2. Completing the presentation for $\mathrm{SU}(3, q)$. We follow [16] with some variations. Our presentation for $G$ is

$$
\left\{\nu, \tau, \Delta, t \mid R(1), R(2), \Delta^{t}=\Delta^{-q}, t^{2}=1\right\}
$$

where $R(1)$ are the relations in a presentation for $H$ on $\{\nu, \tau, \Delta\}$, and $R(2)$ is to be specified. We omit the relation $\Delta^{q^{2}-1}=1$ from $R(1)$ : it is an obvious consequence of $\Delta^{t}=\Delta^{-q}$ and $t^{2}=1$. Since our defining relations for $H$ are satisfied in $\mathrm{SU}(3, q)$, we may take $H$ to be a subgroup of $G$. The relations $R(2)$ are of the form $u^{t}=u_{L} d t u_{R}$ for $u, u_{L}, u_{R} \in U$, and $d$ in $D:=\langle\Delta\rangle$; in more detail, if $u=\nu(\alpha, \beta)$ and $\beta \neq 0$, so $u \neq 1$, then $d=\operatorname{diag}\left(\beta^{-q}, \beta^{q-1}, \beta\right)$, $u_{L}=\nu\left(-\alpha \beta^{-q}, \beta^{-1}\right)$, and $u_{R}=\nu\left(-\alpha \beta^{-1}, \beta^{-1}\right)$. Note that $\mathrm{SU}(3, q)$ is the disjoint union of $U D t U$ and $H$. Thus $U D t U$ is the set of elements that move the isotropic projective point $[0,0,1]$, and $H$ is the set of elements that fix $[0,0,1]$. Hence it suffices to find relations $R(2)$ that imply that every element of $G$ lies in $U D t U \cup H$, where we abuse notation by using the same letter for $t$ as an element of $G$, and as a matrix. We denote the relation $u^{t}=u_{L} d t u_{R}$ by $P(u)$.

Let $V$ denote the set of $u \in U \backslash 1_{G}$ for which the relation $P(u)$ may be deduced from the above presentation for a given choice of $R(2)$.

Lemma 4.4. If $V=U \backslash\{1\}$ then $G$ is isomorphic to $\mathrm{SU}(3, q)$.

Proof. It is easy to see from these relations that the product of two elements of $G$ of the form $u_{L} d t u_{R}$ can again be written in this form, or as an element of $H$. Thus the union of these subsets of $G$ is a subgroup that contains the generators of $G$. Hence $G$ is equal to this union, and so is isomorphic to $\mathrm{SU}(3, q)$.

Lemma 4.5. $V$ is closed under conjugation by $\Delta$. If $u$ and $v$ lie in $V$ then $u v \in V$ if and only if $u_{R} v_{L} \in V$.

Proof. Observe that $\left(u^{\Delta}\right)^{t}=\left(u^{t}\right)^{\left(\Delta^{t}\right)}$, and $\Delta^{t}=\Delta^{-q}$. Also $t^{\Delta}=\left(\Delta^{-1} \Delta^{t}\right) t$. The result follows.

Lemma 4.6. If $u$ and $v$ lie in $V$ then $u v \in V$ if and only if $u_{R} v_{L} \in V$.

Proof. This is simply a rearrangement of terms.

Lemma 4.7. Let $\beta \in \mathrm{GF}\left(q^{2}\right)$ and let $\eta \in \mathrm{GF}\left(q^{2}\right)$ with $\operatorname{tr}(\eta) \neq 0 \neq \operatorname{tr}(\beta)$. There exists $\gamma \in \operatorname{GF}\left(q^{2}\right)^{\times}$with $\operatorname{tr}(\gamma)=0$, and $\beta+\gamma \in \operatorname{GF}(q)^{\times} \eta$.

Proof. Let $t=\operatorname{tr}(\beta) \operatorname{tr}(\eta)^{-1}$, and $\gamma=t \eta-\beta$.

Theorem 4.8. Suppose that $q \not \equiv 2 \bmod 3$. Let $\beta_{0}=\omega \zeta=\omega^{1+\left(q^{2}+q\right) / 2}$. Pick $\alpha_{0}$ such that $\alpha_{0}^{q+1}=-\operatorname{tr}\left(\beta_{0}\right)$. By Lemma 4.7 there exists $\gamma_{0}$ with $\operatorname{tr}\left(\gamma_{0}\right)=0$ and $\beta_{0}+\gamma_{0} \in \operatorname{GF}(q)^{\times} \omega^{-1} \zeta$. Let $\nu_{0}=\nu\left(\alpha_{0}, \beta_{0}\right)$, let $\tau_{0}=\nu\left(0, \gamma_{0}\right)$, and let $U_{0}=\left\{\nu_{0}, \tau_{0}, \nu_{0} \tau_{0}\right\}$. If $R(2)=\left\{P(u): u \in U_{0}\right\}$ then $G$ is isomorphic to $\mathrm{SU}(3, q)$.

Proof. Note that $\beta_{0}$ is chosen so that $\beta_{0} \zeta \in \mathrm{GF}(q)^{\times} \omega$, so $\beta_{0} \gamma \in \mathrm{GF}(q)^{\times} \omega$ for any $\gamma$ in $\mathrm{GF}\left(q^{2}\right)$ of trace 0 , since $\operatorname{tr}(\zeta)=0$.

It suffices to prove that this choice of $R(2)$ implies that $V=U \backslash\{1\}$.

Let $U_{i}=\left\{\nu(\alpha, \beta) \mid \beta \in \operatorname{GF}(q)^{\times} \omega^{-i} \zeta, \alpha^{q+1}=-\operatorname{tr}(\beta)\right\}$. So $U \backslash\{1\}$ is the disjoint union of the $U_{i}$, as $i$ runs from 0 to $q$. Moreover $U_{0}$ contains $q-1$ elements, and $U_{i}$ contains 
$q^{2}-1$ elements for $1 \leq i \leq q$. Each $U_{i}$ is normalised by $\langle\Delta\rangle$, the action being transitive in all cases.

We prove, by induction on $i$, that $U_{i}$ is contained in $V$ for $0 \leq i \leq q$. The case $i=0$ holds, since $\tau_{0} \in V$, and the case $i=1$ holds, since $\nu_{0} \tau_{0}=\nu\left(\alpha_{0}, \beta_{0}+\gamma_{0}\right) \in V$. Now assume inductively that $U_{i}$ is contained in $V$, where $0<i<q$. By Lemma 4.7 there exists $\gamma \in \operatorname{GF}\left(q^{2}\right)$ such that $\operatorname{tr}(\gamma)=0$ and $\beta_{0}+\gamma \in \operatorname{GF}(q)^{\times} \omega^{-i} \zeta$; for, since $\operatorname{tr}(\zeta)=0$, the restriction on $i$ implies that the elements of this coset of $\operatorname{GF}(q)^{\times}$do not have trace 0 . So $\nu\left(\alpha_{0}, \beta_{0}+\gamma\right)$ lies in $V$, by the induction hypothesis, as do $\nu\left(\alpha_{0}, \beta_{0}\right)$ and $\nu(0, \gamma)$. Lemma 4.6 implies that $\nu\left(-\alpha \beta_{0}^{-q}, \beta_{0}^{-1}+\gamma^{-1}\right) \in V$. But $\beta_{0}^{-1}+\gamma^{-1}=\left(\beta_{0}+\gamma\right) / \beta_{0} \gamma \in$ $\operatorname{GF}(q)^{\times} \omega^{-i} \zeta \omega^{-1}=\operatorname{GF}(q)^{\times} \omega^{-(i+1)} \zeta$. (Note that if $\operatorname{tr}(\gamma)=0$ then $\operatorname{tr}\left(\gamma^{-1}\right)=0$.) The result follows.

Theorem 4.9. Suppose that $q \equiv 2 \bmod 3$. Let $\beta_{0}=\omega^{1+\left(q^{2}+q\right) / 2}$. For $0 \leq i \leq 2$ pick $\alpha_{i} \in$ $\omega^{i}\left(\mathrm{GF}\left(q^{2}\right)^{\times}\right)^{3}$ such that $\alpha_{i}^{q+1}=-\operatorname{tr}\left(\beta_{0}\right)$. By Lemma 4.7 there exists $\gamma_{0}$ with $\operatorname{tr}\left(\gamma_{0}\right)=0$ and $\beta_{0}+\gamma_{0} \in \operatorname{GF}(q)^{\times} \omega^{-1} \zeta$. Let $\nu_{i}=\nu\left(\alpha_{i}, \beta_{0}\right)$, let $\tau_{0}=\nu\left(0, \gamma_{0}\right)$, and let $U_{0}=\left\{\nu_{i}, \tau_{0}, \nu_{i} \tau_{0}\right.$ : $0 \leq i \leq 2\}$. If $R(2)=\left\{P(u): u \in U_{0}\right\}$ then $G$ is isomorphic to $\mathrm{SU}(3, q)$.

Proof. Note that $\omega^{q-1}$ is an element of $\operatorname{GF}\left(q^{2}\right)^{\times}$that has norm 1 and is not a cube. If $\alpha^{q+1}=-\operatorname{tr}\left(\beta_{0}\right)$ then $\alpha$ and $\alpha \omega^{q-1}$ and $\alpha \omega^{2(q-1)}$ all have norm equal to $-\operatorname{tr}\left(\beta_{0}\right)$, and lie in different cosets of $\left(\operatorname{GF}\left(q^{2}\right)^{\times}\right)^{3}$ in $\operatorname{GF}\left(q^{2}\right)^{\times}$; so they may be taken to be the elements $\alpha_{i}$ of the theorem.

Define $U_{i}$, where $0 \leq i \leq q$, as in the proof of Theorem 4.8, Again $\langle\Delta\rangle$ acts transitively on $U_{0}$, but partitions $U_{i}$, for $1 \leq 1 \leq q$, into three orbits, the orbit to which a given element $\nu(\alpha, \beta)$ of $U_{i}$ belongs depending on the coset of $\left(\operatorname{GF}\left(q^{2}\right)^{\times}\right)^{3}$ in $\operatorname{GF}\left(q^{2}\right)^{\times}$to which $\alpha$ belongs.

The proof now follows that of Theorem 4.8.

4.2.3. The remaining cases. A presentation for $\mathrm{SU}(3,3)$ is the following:

$$
\begin{aligned}
&\left\{\nu, \tau, \Delta, t \quad \mid \quad \nu^{3}=1, t^{2}=1, \Delta^{-1} \nu^{-1} \Delta^{-1} \tau^{-1} \nu \Delta^{2} \nu^{-1}=1, \Delta^{-1} \nu \Delta^{-1} \tau^{-1} \nu^{-1} \Delta^{2} \nu=1,\right. \\
&\left.\tau t \Delta^{-2}(\tau t)^{2}=1, \Delta \tau^{-1} \nu^{-1} t \Delta \tau^{-1} \nu \Delta t \nu \tau^{-1} t=1\right\} .
\end{aligned}
$$

A presentation for $\mathrm{SU}(3,5)$ is the following:

$$
\begin{aligned}
&\left\{\nu, \tau, \Delta, t \quad \mid \quad \nu^{5}=1, \Delta \tau^{2} \Delta^{-1} \tau=1, \Delta^{2} \nu^{2} \Delta^{-2} \nu^{-1}=1, \Delta^{5} t \Delta t=1, \Delta^{-1} \nu t \nu^{-2} t \nu \Delta t=1,\right. \\
&\left.\Delta \nu \Delta^{-1} \tau^{-1} \nu^{-1} \Delta \nu^{-1} \Delta^{-1} \nu=1, \nu^{-1} t \tau^{-1} \nu^{-1} t \Delta^{-1} \nu^{2} t \Delta \nu^{-1} t \Delta \tau^{-1}=1\right\} .
\end{aligned}
$$

Their correctness is readily established using coset enumeration.

4.3. A presentation for $\mathrm{SU}(3,2)$. We use defining relations for this (soluble) group in our presentations for $\mathrm{SU}(2 n+1,2)$ when $n>1$. Our chosen generating set is $\{\nu=$ $\left.\nu(1, \omega), \nu^{\prime}=\nu\left(\omega^{2}, \omega\right), \Delta=\Delta(\omega), t\right\}$ where $\omega$ is a primitive element for $\operatorname{GF}(4)$. Observe that $\mathrm{SU}(3,2)$ is a split extension of a normal subgroup isomorphic to the group of order 27 and exponent 3 by $Q_{8}$. This structure motivates the following presentation:

$$
\begin{gathered}
\left\{\nu, \nu^{\prime}, \Delta, t \quad \mid \quad a:=[\nu, t], b:=a^{2 \nu}, a^{\nu}=b^{-1}, b^{\nu}=a \Delta, a^{\nu^{\prime}}=a b a, b^{\nu^{\prime}}=a b \Delta,\right. \\
\left.\nu^{2}=\nu^{\prime 2}=\left[\nu, \nu^{\prime}\right], t=\nu^{2} a^{2} b\right\} .
\end{gathered}
$$

Its correctness is readily established using coset enumeration. 
4.4. A presentation for $\operatorname{PSU}(3, q)$. The centre of $\mathrm{SU}(3, q)$ has order $\operatorname{gcd}(q+1,3)$; if it is non-trivial, then it is generated by $\Delta^{\left(q^{2}-1\right) / 3}$.

4.5. Standard generators for $\mathrm{SU}(3, q)$. In Table 1 of [10, 21] the non-trivial standard generators for $\mathrm{SU}(3, q)$ are labelled $s, t, \delta, x, y$. Observe that $t=\tau ; \delta=\Delta^{q+1} ; x=\nu$; $y=\Delta$; if $q$ is odd, then the standard generator $s=\Delta^{(q+1) / 2} t$, else $s=t$. If $q$ is odd, then the presentation generator $t=y^{-(q+1) / 2} s$, else $t=s$.

\section{A presentation for $\operatorname{SL}(d, q)$ FOR $d>2$}

5.1. Generators and notation. Let $q=p^{e}$ for a prime $p$. Let $\omega$ be a primitive element of $\mathrm{GF}(q)$.

Let $d>2$. We take a basis $\left(e_{1}, e_{2}, \ldots, e_{d}\right)$ of the natural module. We define the following elements of $\mathrm{GL}(d, q)$, where $1 \leq i, j \leq d$ and $i \neq j$, and $s \in \mathrm{GF}(q)$.

$$
\begin{aligned}
& \tau_{i j}(s)=\left(e_{i} \mapsto e_{i}+s e_{j}\right) ; \\
& \tau_{i,-j}(s)=\tau_{-i, j}(s)=\tau_{i j}(-s) ; \\
& \tau_{-i,-j}(s)=\tau_{i j}(s) ; \\
& \delta_{i j}=\left(e_{i} \mapsto \omega^{-1} e_{i}, e_{j} \mapsto \omega e_{j}\right) ; \\
& U=\left(e_{1}, e_{2}\right) ; \\
& V=\left(e_{1}, e_{2}, \ldots, e_{d}\right) ; \\
& U^{\prime}=\left(e_{1}, e_{2}\right)^{-} ; \\
& V^{\prime}=\left(e_{1}, e_{2}, \ldots, e_{d}\right)^{\epsilon_{d}}
\end{aligned}
$$

All these elements of $\operatorname{GL}(d, q)$ centralise those basis elements that they are not stated to move. All lie in $\operatorname{SL}(d, q)$ except for $U$ when $q$ is odd, and $V$ when $q$ is odd and $d$ is even. If $q$ is even, then $U^{\prime}=U$ and $V^{\prime}=V$.

5.2. A presentation for $\operatorname{SL}(d, q)$ for $e>1$. We give a presentation for $\operatorname{SL}(d, q)$ on the generating set $\left\{\tau=\tau_{12}(1), \delta=\delta_{12}, U^{\prime}, V^{\prime}\right\}$.

Let $N$ be the subgroup of $\mathrm{SL}(d, q)$ generated by $\left\{\delta, U^{\prime}, V^{\prime}\right\}$. So $N$ is an extension of the direct product of $d-1$ copies of $C_{q-1}$, generated by $\left\{\delta_{12}, \delta_{23}, \ldots, \delta_{d-1, d}\right\}$, by a copy of $S_{d}$, and has order $(q-1)^{d-1} d !$.

Theorem 5.1. Let $G$ be the group generated by $\left\{\delta, U^{\prime}, V^{\prime}\right\}$ subject to the relations given below. Then $G$ is isomorphic to $N$.

(i) Defining relations for $\left\langle U^{\prime}, V^{\prime}\right\rangle$ as signed permutations of $\{1,2, \ldots, d\}$ if $q$ is odd, or as unsigned permutations if $q$ is even.

(ii) $\left[\delta, U^{\prime V^{\prime 2}}\right]=1$ if $d>3$, and $\left[\delta, V^{\prime} U^{\prime} U^{\prime V^{\prime}}\right]=1$ if $d>4$.

(iii) $\delta \delta^{V^{\prime}}=\delta^{V^{\prime} U^{\prime}}$.

(iv) $\delta^{U^{\prime}}=\delta^{-1}$.

(v) $\left[\delta, \delta^{V^{\prime}}\right]=1$, and if $d>3$ then $\left[\delta, \delta^{V^{\prime 2}}\right]=1$.

(vi) If $q$ is even, then $\delta^{q-1}=1$, else $\delta^{(q-1) / 2}=U^{\prime 2}$.

Proof. Since $q \geq 4$, the elements $\delta_{i j}$ are distinct. The centraliser of $\delta$ in $\left\langle U^{\prime}, V^{\prime}\right\rangle$ is generated by the subgroup that, as signed permutation group, fixes 1 and 2 , and by $U^{\prime 2}$ and $U^{\prime 2 V^{\prime}}$ if $q$ is odd. For these elements centralise $\delta$, and generate a subgroup of $\left\langle U^{\prime}, V^{\prime}\right\rangle$ of the appropriate index. But $U^{\prime 2} \in\langle\delta\rangle$, and $U^{\prime 2 V^{\prime}} \in\left\langle\delta^{V^{\prime}}\right\rangle$, so, by (ii) and (v), we may 
define $\delta_{i j}$ in $G$, these elements being permuted by the natural action of $\left\langle U^{\prime}, V^{\prime}\right\rangle$ acting as unsigned permutations. By (v) $\delta_{12}$ commutes with $\delta_{23}$, and with $\delta_{34}$ if $d>3$; so all $\delta_{i j}$ commute with each other. By (iv) $\delta_{12}=\delta_{21}^{-1}$, so $\delta_{i j}=\delta_{j i}^{-1}$ for all $i \neq j$. By (iii) $\delta_{12} \delta_{23}=\delta_{13}$, so $\delta_{i j} \delta_{j k}=\delta_{i k}$ for all $i \neq j \neq k \neq i$. By (vi) $\delta_{12}^{q-1}=1$, so $\delta_{i j}^{q-1}=1$ for all $i \neq j$. Thus $G$ has order at most $(q-1)^{d-1} d$ !. But $N$ is a homomorphic image of $G$ of this order, so $G$ is isomorphic to $N$, as required.

Theorem 5.2. The centraliser of $\tau=\tau_{12}(1)$ in $N$ has index $(q-1) d(d-1)$ in $N$ and is generated by the following elements:

(i) $U^{\prime V^{\prime 2}}$ if $d>3$;

(ii) $V^{\prime} U^{\prime} U^{\prime V^{\prime}}$ if $d>4$;

(iii) $\delta^{V^{\prime 2}}$ if $d>3$;

(iv) $\delta \delta^{2 V^{\prime}}$.

Proof. Clearly $N$ acts transitively by conjugation on $\left\{\tau_{i j}(s) \mid s \in \mathrm{GF}(q)^{\times}\right\}$since $d>2$. Moreover $U^{\prime V^{\prime 2}}$ (if $d>3$ ) and $V^{\prime} U^{\prime} U^{\prime V^{\prime}}$ (if $d>4$ ) stand for the signed permutation matrices $(3,4)^{-}$and $(1)^{-}(2)^{-}(3,4, \ldots, d)^{\epsilon_{d}}$ respectively, and $\delta \delta^{2 V^{\prime}}$ and $\delta^{V^{\prime 2}}$ (if $d>3$ ) stand for the matrices $\operatorname{diag}\left(\omega^{-1}, \omega^{-1}, \omega^{2}, 1, \ldots, 1\right)$ and $\operatorname{diag}\left(1,1, \omega^{-1}, \omega, 1, \ldots, 1\right)$ respectively. These elements centralise $\tau$, and generate a subgroup of $N$ of order $(q-1)^{d-2}(d-2)$ !.

Theorem 5.3. Let $q=p^{e}$ for a prime $p$ and $e>1$, and let $d>2$. Let $G$ be the group generated by $\left\{\tau, \delta, U^{\prime}, V^{\prime}\right\}$ subject to the relations given below. Then $G$ is isomorphic to $\mathrm{SL}(d, q)$.

(i) Defining relations for $N=\left\langle\delta, U^{\prime}, V^{\prime}\right\rangle$ as in Theorem 5.1, but omitting relations (iv) and (vi) of that theorem.

(ii) Relations that present $\mathrm{SL}(2, q)$ on $\left\{\tau, \delta, U^{\prime}\right\}$.

(iii) Relations that state that the elements listed in Theorem 5.2 centralise $\tau$.

(iv) The following instances of Steinberg relations:

(a) $\left[\tau, \tau^{V^{\prime}}\right]=\tau^{U^{\prime V^{\prime}}} ; \quad\left(\left[\tau_{12}(1), \tau_{23}(1)\right]=\tau_{13}(1)\right)$;

(b) $\left[\tau, \tau^{U^{\prime V^{\prime}}}\right]=1 ; \quad\left(\left[\tau_{12}(1), \tau_{13}(1)\right]=1\right)$;

(c) $\left[\tau, \tau^{U^{\prime} V^{\prime}}\right]=1 ; \quad\left(\left[\tau_{12}(1), \tau_{32}(-1)\right]=1\right)$;

(d) if $d>3$ then $\left[\tau, \tau^{V^{\prime 2}}\right]=1 ; \quad\left(\left[\tau_{12}(1), \tau_{34}(1)\right]=1\right)$;

(e) if $q=4$ and $d=3$ then $\left[\tau, \tau^{\delta V^{\prime}}\right]=\tau^{\delta U^{\prime V^{\prime}}} ; \quad\left(\left[\tau_{12}(1), \tau_{23}\left(\omega^{2}\right)\right]=\tau_{13}\left(\omega^{2}\right)\right)$;

(f) if $q=4$ and $d=3$ then $\left[\tau, \tau^{\delta U^{\prime V^{\prime}}}\right]=1 ; \quad\left(\left[\tau_{12}(1), \tau_{13}\left(\omega^{2}\right)\right]=1\right)$;

(g) if $q=4$ and $d=3$ then $\left[\tau, \tau^{\delta U^{\prime} V^{\prime}}\right]=1 ; \quad\left(\left[\tau_{12}(1), \tau_{32}\left(\omega^{2}\right)\right]=1\right)$.

Proof. The omitted relations follow from (ii).

By (iii) the elements $\tau_{i j}(s)$ (for $s \neq 0$ ) may be uniquely defined in $G$ as $N$-conjugates of $\tau$.

The Steinberg relations for $\operatorname{SL}(d, q)$ are as follows, where $i, j, k$ and $l$ are distinct:

(1) $\tau_{i j}(s) \tau_{i j}(t)=\tau_{i j}(s+t) ; \tau_{i j}(s)^{p}=1$;

(2) if $d>3$ then $\left[\tau_{i j}(s), \tau_{k l}(t)\right]=1$;

(3) $\left[\tau_{i j}(s), \tau_{i k}(t)\right]=1$;

(4) $\left[\tau_{i j}(s), \tau_{k j}(t)\right]=1$; 
(5) $\left[\tau_{i j}(s), \tau_{j k}(t)\right]=\tau_{i k}(s t)$.

Relations (1) hold in $G$ for $i=1$ and $j=2$ from (ii), and for other suffices by conjugation in $N$.

In $\operatorname{SL}(d, q)$, if $d>4$, or if $d=4$ and $q$ is even, then $N$ acts transitively by conjugation on the set of ordered pairs $\left(\tau_{i j}(s), \tau_{k l}(t)\right)$, where $i, j, k, l$ are distinct and $s \neq 0 \neq t$. So in our presentation, for these values of $d$ and $q$, we need one relation of the form $\left[\tau_{i j}(s), \tau_{k l}(t)\right]=1$ with suffices satisfying these conditions. Relation (d) is one such. Thus relations (2) hold in $G$ if $d>4$, or if $q$ is even. If $d=4$ and $q$ is odd then the action of $N$ on these pairs gives rise to two orbits, one containing the pairs $\left(\tau_{i j}(s), \tau_{k l}(t)\right)$ where $s t$ is a square in $\operatorname{GF}(q)^{\times}$, and the other containing the pairs where $s t$ is not a square. Now for fixed $i, j, k, l, t$, the set of $s \in \operatorname{GF}(q)$ for which $\left[\tau_{i j}(s), \tau_{k l}(t)\right]=1$ in $G$ is closed under addition (by (a)), and under multiplication by $\omega^{2}$ (by conjugation with $\delta_{i j}$ ). Since $\omega^{2}$ generates $\operatorname{GF}(q)$ as a field, $\left[\tau_{i j}(s), \tau_{k l}(t)\right]=1$ for all $s$ in $\operatorname{GF}(q)$ provided that this holds for one $s \in \operatorname{GF}(q)^{\times}$, and so (2) holds in $G$.

Similarly, if $d>3$ then $N$ acts transitively by conjugation on the set of pairs $\left(\tau_{i j}(s), \tau_{i k}(t)\right)$, where $i, j, k$ are distinct and $s \neq 0 \neq t$; and we have the relation (b), standing for $\left[\tau_{12}(1), \tau_{13}(1)\right]=1$. But if $d=3$ then the setwise stabiliser of the set of pairs $\left(\tau_{12}(s), \tau_{13}(t)\right)$, with $s \neq 0 \neq t$, is the group of diagonal matrices in $\operatorname{SL}(d, q)$, and this group, which has order $(q-1)^{2}$, acts faithfully unless $q \equiv 1 \bmod 3$, when there is a kernel of order 3 . So if $q \equiv 1 \bmod 3$ then we have three orbits of $N$ on these pairs. For fixed $s \in \operatorname{GF}(q)^{\times}$, let $S$ be the set of $t \in \operatorname{GF}(q)$ such that $\left[\tau_{12}(s), \tau_{13}(t)\right]=1$ in $G$. Now $S$ is closed under addition, and (by conjugation with $\delta_{12} \delta_{23}^{2}$ ) is closed under multiplication by $\omega^{3}$, and hence is a vector space over the field generated by $\omega^{3}$. If $q \neq 4$ then this field is $\operatorname{GF}(q)$, and a similar result holds with the roles of $s$ and $t$ interchanged. Thus, in this case, the relations (3) may be deduced from a single case. But if $q=4$ the field generated by $\omega^{3}$ is the prime field, and we need relation (f), which asserts that $\left[\tau_{12}(1), \tau_{13}\left(\omega^{2}\right)\right]=1$. Hence $\left[\tau_{12}(1), \tau_{13}\left(1+\omega^{2}\right)\right]=1$, so we have representatives of the three orbits of $N$, as above, thus (3) holds in all cases.

By a similar argument, relations (4) may be deduced, using relations (c) and (g).

The proof of (5) is similar. If $q \not \equiv 1 \bmod 3$, or $d>3$, then the result follows from (a) by conjugation with $N$. Otherwise, we first need to prove that in $G$, for given $s \in \operatorname{GF}(q)^{\times}$, the set $S$ consisting of those $t$ for which $\left[\tau_{12}(s), \tau_{23}(t)\right]=\tau_{13}(s t)$ is closed under addition, and under multiplication by $\omega^{3}$. This follows from the identity $[a, b c]=[a, c][a, b]^{c}$, using the relation (a), and by conjugation with $\delta_{12} \delta_{23}^{2}$. This completes the proof of (5), except in the case when $q=4$ and $d=3$, when we use relation (e).

Finally one must check that the given generators lie in the group generated by the $\tau_{i j}(s, t)$. This holds for $\tau, \delta$ and $U^{\prime}$, thus for all $N$-conjugates of $U^{\prime}$, and so for $V^{\prime}$.

Coset enumeration shows that relations (f) and (g) are redundant.

5.3. A presentation for $\mathrm{SL}(d, p)$. We give a presentation for $\mathrm{SL}(d, p)$ on the generating set $\left\{\tau, U^{\prime}, V^{\prime}\right\}$. Clearly $\left\langle U^{\prime}, V^{\prime}\right\rangle$ acts transitively by conjugation on the set of elements $\tau_{i j}( \pm 1)$, a set of size $2 d(d-1)$ if $p$ is odd, and of size $d(d-1)$ if $p=2$. If $p$ is odd, then the centraliser of $\tau$ in $\left\langle U^{\prime}, V^{\prime}\right\rangle$ is the direct product of the cyclic group of order 2 generated by $U^{\prime 2}$ with the subgroup of $\left\langle U^{\prime}, V^{\prime}\right\rangle$ that, as signed permutation group, fixes 1 and 2 . This 
subgroup has index $2 d(d-1)$, as required. If $p=2$, then this centraliser is the subgroup of $\langle U, V\rangle$ that fixes 1 and 2 , and has index $d(d-1)$, as required.

Theorem 5.4. Let $p$ be a prime and let $d>2$. Let $G$ be the group generated by $\left\{\tau, U^{\prime}, V^{\prime}\right\}$ subject to the relations given below. Then $G$ is isomorphic to $\operatorname{SL}(d, p)$.

(i) Defining relations for $\left\langle U^{\prime}, V^{\prime}\right\rangle$ as signed permutations of $\{1,2, \ldots, d\}$ if $p$ is odd, or as unsigned permutations if $p=2$.

(ii) Relations that present $\mathrm{SL}(2, p)$ on $\left\{\tau, U^{\prime}\right\}$.

(iii) If $d>3$ then $\left[\tau, U^{\prime V^{\prime 2}}\right]=1$, and if $d>4$ then $\left[\tau, V^{\prime} U^{\prime-1}\left(U^{\prime-1}\right)^{V^{\prime}}\right]=1$.

(iv) The Steinberg relations (iv)(a) to (iv)(d) of Theorem 5.3 .

Proof. That $U^{\prime 2}$ centralises $\tau$ follows from (ii). Hence, by virtue of (i) and (iii), if $p$ is odd then we may define $\tau_{i j}( \pm 1)$ as elements of $G$ by conjugation with $\left\langle U^{\prime}, V^{\prime}\right\rangle$. The proof is concluded as in Theorem 5.3.

Observe that $\delta=\left(\tau^{\omega-\omega^{2}}\right){ }^{U^{\prime}} \tau^{\omega^{-1}}\left(\tau^{\omega-1}\right)^{U^{\prime}} \tau^{-1}$.

5.4. A presentation for $\operatorname{PSL}(d, q)$. The centre of $\operatorname{SL}(d, q)$ has order $\operatorname{gcd}(q-1, d)$; if it is non-trivial, then it is generated by $\left(\delta U^{\prime} V^{\prime-1}\right)^{(d-1)(q-1) / \operatorname{gcd}(q-1, d)}$. For $\left(\delta U^{\prime} V^{\prime-1}\right)^{d-1}$ is a diagonal matrix whose entries, with one possible exception, equal $-\epsilon_{d} \omega$.

5.5. Standard generators for $\operatorname{SL}(d, q)$. In Table 1 of [10, 21] the non-trivial standard generators for $\mathrm{SL}(d, q)$ are labelled $s, t, \delta, v$ where, for ease of exposition, we make one uniform choice for $v$ : namely,

$$
v:=\left(\begin{array}{cc}
0 & 1 \\
-I_{d-1} & 0
\end{array}\right)
$$

Observe that $s=U^{\prime} ; t=\tau$; the standard generator $\delta$ is the inverse of the presentation generator $\delta$; and $v=\left(V^{\prime} U^{\prime-1}\right)^{d-1} V^{\prime-1}$ if $d$ and $q$ are both odd, else $v=V^{\prime d-1}$. The presentation generator $V^{\prime}=v^{-1}\left(s^{-1} v\right)^{d-1}$ if $d$ and $q$ are odd, else $V^{\prime}=v^{d-1}$.

\section{A PRESEntation FOR $\operatorname{Sp}(2 n, q)$ FOR $n>1$}

6.1. Generators and notation. Let $q=p^{e}$ for a prime $p$. Let $\omega$ be a primitive element of $\operatorname{GF}(q)$.

Let $n>1$. We take a hyperbolic basis $\left(e_{1}, f_{1}, \ldots, e_{n}, f_{n}\right)$ of the natural module, and define the following elements of $\operatorname{Sp}(2 n, q)$, where $1 \leq i, j \leq n$ and $i \neq j$, and $s \in \operatorname{GF}(q)$.

$$
\begin{aligned}
& Z_{i}=\left(e_{i} \mapsto f_{i}, f_{i} \mapsto-e_{i}\right) ; \\
& \delta_{i}=\left(e_{i} \mapsto \omega^{-1} e_{i}, f_{i} \mapsto \omega f_{i}\right) ; \\
& \tau_{i}(s)=\left(e_{i} \mapsto e_{i}+s f_{i}\right) ; \\
& \tau_{-i}(s)=\left(f_{i} \mapsto f_{i}-s e_{i}\right)=\tau_{i}(s)^{Z_{i}} ; \\
& \sigma_{i j}(s)=\left(e_{i} \mapsto e_{i}+s e_{j}, f_{j} \mapsto f_{j}-s f_{i}\right) ; \\
& \sigma_{-i, j}(s)=\left(f_{i} \mapsto f_{i}+s e_{j}, f_{j} \mapsto f_{j}+s e_{i}\right)=\sigma_{i j}(s)^{Z_{i}} ; \\
& \sigma_{i,-j}(s)=\left(e_{i} \mapsto e_{i}+s f_{j}, e_{j} \mapsto e_{j}+s f_{i}\right)=\sigma_{i j}(s)^{Z_{j}} ; \\
& \sigma_{-i,-j}(s)=\left(f_{i} \mapsto f_{i}+s f_{j}, e_{j} \mapsto e_{j}-s e_{i}\right)=\sigma_{j i}(-s)=\sigma_{i j}(s)^{Z_{i} Z_{j}} ; \\
& U=\left(e_{1}, e_{2}\right)\left(f_{1}, f_{2}\right) ; \\
& V=\left(e_{1}, e_{2}, \ldots, e_{n}\right)\left(f_{1}, f_{2}, \ldots, f_{n}\right) \text { if } n>2 .
\end{aligned}
$$


All these elements of $\operatorname{Sp}(2 n, q)$ centralise those basis elements that they are not stated to move. Note that $\sigma_{-i, j}^{Z_{i}}(s)=\sigma_{i,-j}^{Z_{j}}(s)=\sigma_{i j}(-s)$.

6.2. A presentation for $\operatorname{Sp}(2 n, q)$ for $e>1$. We give a presentation for $\operatorname{Sp}(2 n, q)$ on the generating set $\left\{\sigma=\sigma_{12}(1), \tau=\tau_{1}(1), \delta=\delta_{1}, Z=Z_{1}, U, V\right\}$, omitting $V$ if $n=2$.

If $q$ is even, then the subgroup of $\operatorname{Sp}(2 n, q)$ obtained by omitting $\tau$ is $\mathrm{SO}^{+}(2 n, q)$.

Let $N_{1}$ be the subgroup of $\operatorname{Sp}(2 n, q)$ generated by $\{Z, U, V\}$, omitting $V$ if $n=2$. It is isomorphic to $C_{2} 2 S_{n}$ if $q$ is even and to $C_{4} 2 S_{n}$ if $q$ is odd. The following is clear.

Theorem 6.1. Let $G$ be the group generated by $\{Z, U, V\}$, omitting $V$ if $n=2$, subject to the relations given below. Then $G$ is isomorphic to $N_{1}$.

(i) Defining relations for $S_{n}$ on $\{U, V\}$, omitting $V$ if $n=2$.

(ii) $Z^{m}=1$, where $m=2$ if $q$ is even, and $m=4$ if $q$ is odd.

(iii) $\left[Z, U^{V}\right]=1$ if $n>2$, and $[Z, V U]=1$ if $n>3$.

(iv) $\left[Z, Z^{U}\right]=1$.

Let $N$ be the subgroup of $\operatorname{Sp}(2 n, q)$ generated by $\{\delta, Z, U, V\}$. Now $K=\langle\delta, Z\rangle$ is a copy of $D_{2(q-1)}$ if $q$ is even, and of $Q_{2(q-1)}$ if $q$ is odd; and $N$ is isomorphic to $K \imath S_{n}$.

Theorem 6.2. Let $G$ be the group generated by $\{\delta, Z, U, V\}$ subject to the relations given below. Then $G$ is isomorphic to $N$.

(i) Defining relations for $\langle Z, U, V\rangle$, as in Theorem 6.1.

(ii) $\delta^{q-1}=1$ if $q$ is even, and $\delta^{(q-1) / 2}=Z^{2}$ if $q$ is odd, and $\delta^{Z}=\delta^{-1}$.

(iii) If $n>2$ then $\left[\delta, U^{V}\right]=1$, and if $n>3$ then $[\delta, V U]=1$.

(iv) $\left[Z, \delta^{U}\right]=\left[\delta, \delta^{U}\right]=1$.

Proof. Since $U^{V}$ and $V U$ stand for the permutations $(2,3)$ and $(2,3, \ldots, n)$ respectively, the result is clear.

Theorem 6.3. The centraliser of $\sigma=\sigma_{12}(1)$ in $N$ is generated by the following elements:

(i) $Z U Z^{-1}$;

(ii) $U^{V^{2}}$ if $n>3$;

(iii) $V U U^{V}$ if $n>4$;

(iv) $Z^{V^{2}}$ if $n>2$;

(v) $\delta^{V^{2}}$ if $n>2$;

(vi) $\delta \delta^{V}$.

It is isomorphic to $K \times\left(K \backslash S_{n-2}\right)$.

The centraliser of $\tau=\tau_{1}(1)$ in $N$ is generated by the following elements:

(i) $Z^{U}$;

(ii) $U^{V}$ if $n>2$;

(iii) $V U$ if $n>3$;

(iv) $Z^{2}$ if $q$ is odd;

(v) $\delta^{U}$.

If $q$ is even it is isomorphic to $K \backslash S_{n-1}$, else to $\left\langle Z^{2}\right\rangle \times\left(K \backslash S_{n-1}\right)$.

Proof. Observe that $N$ acts transitively by conjugation on the set of $2 n(n-1)(q-1)$ short root elements $\sigma_{i j}(s)$, where $0<|i| \neq|j| \leq n$ and $s \in \mathrm{GF}(q)^{\times}$, bearing in mind that 
$\sigma_{i j}(s)=\sigma_{-j,-i}(-s)$ for any $i, j, s$. The centraliser of $\sigma$ in $N$ contains the direct product of a subgroup that is isomorphic to $K \backslash S_{n-2}$ with another copy of $K$, namely $\left\langle\delta_{1} \delta_{2}, Z U Z^{-1}\right\rangle$. Since this direct product has index $2 n(n-1)(q-1)$ in $N$, it is the centraliser of $\sigma$.

Also, $N$ acts by conjugation on the set of $2 n(q-1)$ long root elements $\tau_{i}(s)$, where $0<|i| \leq n$ and $s \in \operatorname{GF}(q)^{\times}$. The action is transitive if $q$ is even, and there are two orbits of equal size if $q$ is odd, $\tau_{i}(s)$ falling into one or other orbit according as $s$ is or is not a square. The elements $U$ and $V$ permute the suffices, positive or negative, of the root elements $\tau_{i}(s)$ in the natural way, and $Z_{i}$ interchanges the suffices $i$ and $-i$ and fixes all other suffices. If $i>0$ then $\tau_{i}(s)^{\delta_{i}}=\tau_{i}\left(s \omega^{2}\right)$, and $\tau_{-i}(s)^{\delta_{i}}=\tau_{-i}\left(s \omega^{-2}\right)$; and $\delta_{i}$ centralises $\tau_{j}$ if $|j| \neq i$. Thus the stated elements centralise $\tau$, and generate a subgroup of $N$ of index $2 n(q-1)$ if $q$ is even, and $n(q-1)$ if $q$ is odd.

Theorem 6.4. Let $\Delta=[U, \delta]$; and $U^{\prime}=U Z^{2}$ if $q$ is odd, $U^{\prime}=U$ if $q$ is even. The subgroup of $\operatorname{Sp}(2 n, q)$ generated by $\left\{\sigma, \Delta, U^{\prime}\right\}$ is isomorphic to $\operatorname{SL}(2, q)$.

Proof. The isomorphism is obtained by restriction to the subspace $\left\langle e_{1}, e_{2}\right\rangle$, or $\left\langle f_{1}, f_{2}\right\rangle$, these two representations being connected by the inverse transpose automorphism.

Theorem 6.5. Let $q=p^{e}$ for a prime $p$ and $e>1$, and let $n \geq 2$. Let $G$ be the group generated by $\{\sigma, \tau, \delta, Z, U, V\}$, omitting $V$ if $n=2$, subject to the relations given below. Then $G$ is isomorphic to $\operatorname{Sp}(2 n, q)$.

(i) Defining relations for $N=\langle\delta, Z, U, V\rangle$, omitting $V$ if $n=2$, as in Theorem 6.2, but omitting relations (ii) of that theorem, and relation (ii) of Theorem 6.1.

(ii) Relations that state that the elements listed in Theorem 6.3 centralise $\sigma$ and $\tau$, but omitting the relation $[\tau, V U]=1$ if $q$ is odd.

(iii) Relations that present $\mathrm{SL}(2, q)$ on $\left\{\sigma, \Delta, U^{\prime}\right\}$ as in Theorem 6.4.

(iv) Relations that present $\mathrm{SL}(2, q)$ on $\{\tau, \delta, Z\}$.

(v) The following instances of Steinberg relations:

(a) if $n>2$ then $\left[\sigma, \sigma^{V}\right]=\sigma^{V U} ; \quad\left(\left[\sigma_{i j}(s), \sigma_{j k}(t)\right]=\sigma_{i k}(s t)\right)$;

(b) if $n>2$ then $\left[\sigma, \sigma^{V U}\right]=1 ; \quad\left(\left[\sigma_{i j}(s), \sigma_{i k}(t)\right]=1\right)$;

(c) if $n>2$ then $\left[\sigma, \sigma^{U V}\right]=1 ; \quad\left(\left[\sigma_{i j}(s), \sigma_{k j}(t)\right]=1\right)$;

(d) if $n>3$ then $\left[\sigma, \sigma^{V^{2}}\right]=1 ; \quad\left(\left[\sigma_{i j}(s), \sigma_{k l}(t)\right]=1\right)$;

(e) if $q$ is odd then $\left[\sigma, \sigma^{Z}\right]=\tau^{2 Z U}$, else $\left[\sigma, \sigma^{Z}\right]=1$;

$\left(\left[\sigma_{i j}(s), \sigma_{-i, j}(t)\right]=\tau_{-j}(2 s t)\right)$

(f) $[\sigma, \tau]=1 ; \quad\left(\left[\sigma_{i j}(s), \tau_{i}(t)\right]=1\right)$;

(g) $\left[\sigma, \tau^{U}\right]=\sigma^{Z^{U}} \tau^{-1} ; \quad\left(\left[\sigma_{i j}(s), \tau_{j}(t)\right]=\sigma_{i,-j}(s t) \tau_{i}\left(-s^{2} t\right)\right)$;

(h) if $n>2$ then $\left[\sigma, \tau^{V^{2}}\right]=1 ; \quad\left(\left[\sigma_{i j}(s), \tau_{k}(t)\right]=1\right)$;

(j) $\left[\tau, \tau^{U}\right]=1 ; \quad\left(\left[\tau_{i}(s), \tau_{j}(t)\right]=1\right)$.

The given relations are instances of the more general relations in parentheses, with $s=t=1$ and $(i, j, k, l)=(1,2,3,4)$. In the parenthetical version, the moduli of $i, j, k, l$ are distinct.

Proof. The double suffices $(i, j)$ for $\sigma$ and the suffices $i$ for $\tau$ correspond to short and long roots respectively; and if $r$ is the root corresponding to $(i, j)$ or $i$, then $-r$ is the root corresponding to $(-i,-j)$ (equivalently to $(j, i))$ or $-i$ respectively.

The relations omitted from (i) follow from (iv). 
By (i) we may define the elements $\left\{\delta_{i}, Z_{i}, U, V\right\}$ in $G$, and hence also the elements $\Delta$ and $U^{\prime}$.

By Theorem 6.3, and using (i) and (ii), we may define $\left\{\sigma_{i j}(s)|0<| i|\neq| j \mid \leq n, s \in\right.$ $\left.\operatorname{GF}(q)^{\times}\right\}$, as a subset of $G$.

The relation $[\tau, V U]=1$, omitted from (ii) if $q$ is odd, may now be supplied as follows. It follows from (v)(e) that the subgroup of $\langle U, V\rangle$ that centralises 1 and 2 , as permutations of $\{1,2, \ldots, n\}$, centralises $\tau$ as a subgroup of $G$. But this subgroup, together with $U^{V}$ (see (ii)), generates the symmetric group on $\{2,3, \ldots, n\}$, and hence contains $V U$.

By Theorem 6.3, and using (i) and (ii), we may define $\left\{\tau_{i}(s)|0<| i \mid \leq n, s \in \operatorname{GF}(q)^{2}\right\}$ as a subset of $G$, and obtain the centralisers of these elements in $N$. Suppose now that $q$ is odd. Using (iv) we may define $\tau_{1}(\omega)$ in $G$, and writing $\omega$ as a sum of squares gives $\tau_{1}(\omega)$ as a product of elements of the form $\tau_{i}\left(t^{2}\right)$ for $t \in \mathrm{GF}(q)^{\times}$. But these elements all have the same centraliser $C$ in $N$. So $C$ also centralises $\tau_{1}(\omega)$; since it has the expected index in $N$ it must be the full centraliser. So we may define $\tau_{i}(s)$ for all $i$, and for all $s \in \mathrm{GF}(q) \backslash \mathrm{GF}(q)^{2}$, as $N$-conjugates of $\tau_{1}(\omega)$.

We now turn to the Steinberg relations.

The relations $\sigma_{i j}(s+t)=\sigma_{i j}(s) \sigma_{i j}(t)$ and $\sigma_{i j}(s)^{p}=1$ follow from (ii) and (iii), and the relations $\tau_{i}(s+t)=\tau_{i}(s) \tau_{i}(t)$ and $\tau_{i}(s)^{p}=1$ follow similarly from (ii) and (iv).

The remaining Steinberg relations are (a) to $(\mathrm{j})$, and we prove that the general case, given in parentheses, is implied by the given relation.

The general case of relations (a) to (d) clearly follow from the one given instance by conjugation with $N$.

For fixed $i, j$, and $s$ the set of values $t$ for which the general case of relation (f) holds is closed under addition and multiplication by $\omega^{2}$ (conjugate by $\delta_{i} \delta_{j}$ ) and hence is either $\mathrm{GF}(q)$ or $\{0\}$, since $\omega^{2}$ generates $\mathrm{GF}(q)$ as a field. It follows, from conjugation by $N$, that all instances of (f) follow from the given instance.

Let $P$ be the set of pairs $(s, t)$ for which relation (e) holds for one (and hence every) value of $(i, j)$. Conjugation by $\delta_{i}$ and $\delta_{j}$ shows that if $(s, t) \in P$, then $\left(\omega s, \omega^{-1} t\right) \in P$, and $\left(\omega^{-1} s, \omega t\right) \in P$; so $\left(\omega^{2} s, t\right) \in P$. If $\left(s_{1}, t\right) \in P$ and $\left(s_{2}, t\right) \in P$ then $\left(s_{1}+s_{2}, t\right) \in P$, thanks to (f), which implies that $\left[\sigma_{i j}(s), \tau_{-j}(k)\right]=\left[\sigma_{-i, j}(s), \tau_{-j}(k)\right]=1$. As in the proof of (f), this suffices to prove the general case of $(\mathrm{e})$.

Similarly the general cases of $(\mathrm{h})$ and $(\mathrm{j})$ follow from a single case.

To prove the general case of $(\mathrm{g})$, note that $\tau_{j}(t)$ commutes with the RHS of this identity by (f) and (j), since $\sigma_{i,-j}(s t)=\sigma_{j,-i}(-s t)$. The proof is similar to that of (e).

Variants of (e), (f), and (g) may be obtained by changing signs of suffices, but these follow from the given relations. For example, for $i$ and $j$ positive,

$$
\begin{aligned}
{\left[\sigma_{i j}(s), \tau_{-i}(t)\right]=\left[\sigma_{-i, j}(-s), \tau_{i}(t)\right]^{Z_{i}} } & =\left[\sigma_{-j, i}(s), \tau_{i}(t)\right]^{Z_{i}}=\left[\sigma_{j i}(s), \tau_{i}(t)\right]^{Z_{i} Z_{j}} \\
& =\sigma_{j,-i}(s t) \tau_{j}\left(-s^{2} t\right)=\sigma_{i,-j}(-s t) \tau_{j}\left(-s^{2} t\right)
\end{aligned}
$$

So all the Steinberg relations hold in $G$. Thus the subgroup $H$ of $G$ generated by the Steinberg generators is isomorphic to $\operatorname{Sp}(2 n, q)$. Also, $Z$ and $\delta$ and $U^{\prime}$ lie in this group by (iii) and (iv), and hence $U$ lies in $H$. But $H$ is normalised by $\langle U, V\rangle$, and $\langle U, V\rangle$ is isomorphic to $S_{n}$, by (i), so $V \in H$. Thus $G=H$, as required. 
6.3. A presentation for $\operatorname{Sp}(2 n, p)$. We give a presentation for $\operatorname{Sp}(2 n, p)$ on the generating set $\{\sigma, \tau, Z, U, V\}$, omitting $V$ if $n=2$. Let $N_{1}$ be the subgroup of $\operatorname{Sp}(2 n, p)$ generated by $\{Z, U, V\}$, omitting $V$ if $n=2$.

Theorem 6.6. The centraliser of $\sigma=\sigma_{12}(1)$ in $N_{1}$ is generated by the corresponding elements $(i)-(i v)$ listed in Theorem [6.3, and $\left[Z^{2}, U\right]$ if $p$ is odd. It is isomorphic to $C_{2} \times\left(C_{2} \succ S_{n-2}\right)$ if $p=2$, and to $C_{2} \times C_{2} \times\left(C_{4}\right.$ 乙 $\left.S_{n-2}\right)$ if $p$ is odd.

The centraliser of $\tau=\tau_{1}(1)$ in $N_{1}$ is generated by the corresponding elements $(i)-(i v)$ listed in Theorem 6.3. It is isomorphic to $C_{2}$ 々 $S_{n-1}$ if $p=2$, and to $C_{2} \times\left(C_{4} 2 S_{n-1}\right)$ if $p$ is odd.

Proof. The listed elements clearly generate groups having the claimed isomorphism types. They generate subgroups of $N_{1}$ of index $2 n$ in the case of the centraliser of $\tau$; and of index $4 n(n-1)$ if $p$ is odd, and index $2 n(n-1)$ if $p=2$, in the case of the centraliser of $\sigma$. This corresponds to the fact that $N_{1}$ permutes transitively the $2 n$ elements $\tau_{ \pm i}(1)$ with $1 \leq i \leq n$, and the $4 n(n-1)$ elements $\sigma_{ \pm i, \pm j}( \pm 1)$ with $1 \leq i \neq j \leq n$ if $p$ is odd, and the $2 n(n-1)$ elements $\sigma_{ \pm i, \pm j}(1)$ with $1 \leq i \neq j \leq n$ if $p=2$. (Recall that, in either characteristic, these elements are equal in pairs.)

Theorem 6.7. Let $p$ be a prime and let $n \geq 2$. Let $G$ be the group generated by $\{\sigma, \tau, Z, U, V\}$, omitting $V$ if $n=2$, subject to the relations given below. Then $G$ is isomorphic to $\operatorname{Sp}(2 n, p)$.

(i) Defining relations for $N_{1}=\langle Z, U, V\rangle$ as in Theorem 6.1 .

(ii) Relations that state that the elements listed in Theorem 6.6 centralise $\sigma$ and $\tau$, but omitting the relation $[\tau, V U]=1$ if $p$ is odd.

(iii) Relations that present $\mathrm{SL}(2, p)$ on $\left\{\sigma, U^{\prime}\right\}$.

(iv) Relations that present $\mathrm{SL}(2, p)$ on $\{\tau, Z\}$.

(v) The Steinberg relations (v) of Theorem 6.5.

Proof. The proof is similar to that of Theorem 6.5.

Observe that $\delta=\left(\tau^{\omega-\omega^{2}}\right)^{Z} \tau^{\omega^{-1}}\left(\tau^{\omega-1}\right)^{Z} \tau^{-1}$.

6.4. A presentation for $\operatorname{PSp}(2 n, q)$. If $q$ is even, then $\operatorname{Sp}(2 n, q)$ is simple. If $q$ is odd, then the centre of $\operatorname{Sp}(2 n, q)$ has order 2 , and is generated by $(Z V)^{2 n}$.

6.5. Standard generators for $\operatorname{Sp}(2 n, q)$. In Table 1 of [10, 21] the standard generators for $\operatorname{Sp}(d, q)$ are labelled $s, t, \delta, u, v, x$. Observe that $s=Z ; t=\tau ; u=U ; v=V ; x=\sigma^{Z}$; and the standard generator $\delta$ is the inverse of the presentation generator $\delta$.

\section{A presentation FOR $\mathrm{SU}(2 n, q)$ FOR $n>1$}

7.1. Generators and notation. Let $q=p^{e}$ for a prime $p$. Let $\omega$ be a primitive element of $\operatorname{GF}\left(q^{2}\right)$, and let $\omega_{0}=\omega^{q+1}$, so $\omega_{0}$ is a primitive element of $\operatorname{GF}(q)$. Define $\psi=\omega^{(q+1) / 2}$ if $q$ is odd, and $\psi=1$ if $q$ is even.

Let $n>1$. We take a hyperbolic basis $\left(e_{1}, f_{1}, \ldots, e_{n}, f_{n}\right)$ of the natural module. We define the following elements of $\mathrm{SU}(2 n, q)$, where $1 \leq i, j \leq n$ and $i \neq j$, and $s \in \mathrm{GF}(q)$, and $\alpha \in \operatorname{GF}\left(q^{2}\right)$.

$$
Z_{i}=\left(e_{i} \mapsto-\psi f_{i}, f_{i} \mapsto \psi^{-1} e_{i}\right)
$$




$$
\begin{aligned}
& \Delta_{i j}=\left(e_{i} \mapsto \omega^{-1} e_{i}, f_{i} \mapsto \omega^{q} f_{i}, e_{j} \mapsto \omega e_{j}, f_{j} \mapsto \omega^{-q} f_{j}\right) ; \\
& \delta_{i}=\left(e_{i} \mapsto \omega_{0}^{-1} e_{i}, f_{i} \mapsto \omega_{0} f_{i}\right) ; \\
& \tau_{i}(s)=\left(e_{i} \mapsto e_{i}-s \psi f_{i}\right) ; \\
& \tau_{-i}(s)=\left(f_{i} \mapsto f_{i}+s \psi^{-1} e_{i}\right)=\tau_{i}(s)^{Z_{i}} ; \\
& \sigma_{i j}(\alpha)=\left(e_{i} \mapsto e_{i}+\alpha e_{j}, f_{j} \mapsto f_{j}-\alpha^{q} f_{i}\right) ; \\
& \sigma_{-i, j}(\alpha)=\left(f_{i} \mapsto f_{i}-\alpha \psi^{-1} e_{j}, f_{j} \mapsto f_{j}-\alpha^{q} \psi^{-1} e_{i}\right)=\sigma_{i j}(\alpha)^{Z_{i}}=\sigma_{-j, i}\left(\alpha^{q}\right) ; \\
& \sigma_{i,-j}(\alpha)=\left(e_{i} \mapsto e_{i}-\alpha \psi f_{j}, e_{j} \mapsto e_{j}-\alpha^{q} \psi f_{i}\right)=\sigma_{i j}(\alpha)^{Z_{j}}=\sigma_{j,-i}\left(\alpha^{q}\right) ; \\
& \sigma_{-i,-j}(\alpha)=\left(f_{i} \mapsto f_{i}+\alpha f_{j}, e_{j} \mapsto e_{j}-\alpha^{q} e_{i}\right)=\sigma_{i j}(\alpha)^{Z_{i} Z_{j}}=\sigma_{j i}\left(-\alpha^{q}\right) ; \\
& U=\left(e_{1}, e_{2}\right)\left(f_{1}, f_{2}\right) ; \\
& V=\left(e_{1}, e_{2}, \ldots, e_{n}\right)\left(f_{1}, f_{2}, \ldots, f_{n}\right) \text { if } n>2 .
\end{aligned}
$$

All these elements of $\mathrm{SU}(2 n, q)$ centralise those basis elements that they are not stated to move. As in the case of $\operatorname{Sp}(2 n, q), \sigma_{i j}(\alpha)^{Z_{i}^{2}}=\sigma_{i j}(-\alpha)=\sigma_{i j}(\alpha)^{Z_{j}^{2}}$. Note that the change of basis that fixes $e_{i}$ and sends $f_{i}$ to $-\psi^{-1} f_{i}$ for all $i$ has the effect of replacing $\psi$ by 1 in these definitions. Thus the isomorphism type of the group generated by these elements is independent of $\psi$. So the value of $\psi$ plays no role in the presentation and proofs, apart from the fact that $\psi \neq 0$. We choose $\psi$ to ensure that these elements preserve the given form.

7.2. A presentation for $\mathrm{SU}(2 n, q)$. We give a presentation for $\mathrm{SU}(2 n, q)$ on the generating set $\left\{\sigma=\sigma_{12}(1), \tau=\tau_{1}(1), Z=Z_{1}, \delta=\delta_{1}, \Delta=\Delta_{12}, U=U_{12}, V\right\}$, omitting $V$ if $n=2$. Note that $\delta=\Delta \Delta^{-Z}$. If $q=2$ then $\delta=1$, permitting obvious simplifications to the listed presentations.

Let $H$ be the subgroup of $\mathrm{SU}(2 n, q)$ generated by $\{\Delta, U, V\}$, omitting $V$ if $n=2$. Clearly $H$ has order $\left(q^{2}-1\right)^{n-1} n$ !.

Theorem 7.1. Let $n \geq 2$ and let $G$ be the group generated by $\{\Delta, U, V\}$, omitting $V$ if $n=2$, subject to the relations given below. Then $G$ is isomorphic to $H$.

(i) Defining relations for $S_{n}$ on $\{U, V\}$.

(ii) If $n>3$ then $\left[\Delta, U^{V^{2}}\right]=1$, and if $n>4$ then $\left[\Delta, V U U^{V}\right]=1$.

(iii) $\Delta^{U}=\Delta^{-1}$.

(iv) $\Delta \Delta^{V}=\Delta^{V U}$.

(v) $\left[\Delta, \Delta^{V}\right]=1$, and if $n>3$ then $\left[\Delta, \Delta^{V^{2}}\right]=1$.

(vi) $\Delta^{q^{2}-1}=1$.

We omit those relations that involve $V$ if $n=2$.

Proof. The result is clear if $n=2$, so assume $n>2$. Relations (ii) state that $\Delta$ is centralised by the subgroup of $\langle U, V\rangle$ that corresponds to the copy of $S_{n-2}$ that fixes 1 and 2 , so the conjugates of $\Delta$ under $\langle U, V\rangle$ may be labelled as $\Delta_{i j}$, where $1 \leq i \neq j \leq n$. Relation (iii) states that $\Delta_{j i}=\Delta_{i j}^{-1}$ when $(i, j)=(1,2)$, and hence for any suffices $(i, j)$. Relation (v) states that $\Delta_{12}$ commutes with $\Delta_{23}$ and $\Delta_{34}$, when these are defined, and hence all $\Delta_{i j}$ commute with each other. Finally relation (iv) states that $\Delta_{i, i+1} \Delta_{i+1, i+2}=$ $\Delta_{i, i+2}$ when $i=1$, and hence for all $i$. Hence, in $G$, every element of the group generated by the $\Delta_{i j}$ may be written in the form $\Delta_{12}^{m_{1}} \Delta_{23}^{m_{2}} \cdots \Delta_{n-1, n}^{m_{n-1}}$, and the theorem is proved.

Let $K$ be the subgroup of $\mathrm{SU}(2 n, q)$ generated by $\{\delta, \Delta, U, V\}$, omitting $V$ if $n=2$. Clearly $K$ has order $(q-1)\left(q^{2}-1\right)^{n-1} n !$. 
Theorem 7.2. Let $G$ be the group generated by $\{\delta, \Delta, U, V\}$, omitting $V$ if $n=2$, subject to the relations listed below. Then $G$ is isomorphic to $K$.

(i) Defining relations for $\langle\Delta, U, V\rangle$ as in Theorem 7.1 , omitting $V$ if $n=2$.

(ii) $\delta^{q-1}=1$.

(iii) $\left[\delta, U^{V}\right]=1$, and if $n>3$ then $[\delta, V U]=1$.

(iv) $[\delta, \Delta]=1$, and $\left[\delta, \Delta^{V}\right]=1$.

(v) $\Delta^{q+1}=\delta \delta^{-U}$.

We omit those relations that involve $V$ if $n=2$.

Proof. Suppose first that $n>2$. Relations (iii) state that $\delta$ is centralised by the subgroup of $\langle U, V\rangle$ that corresponds to the subgroup of $S_{n-1}$ that fixes 1, so the conjugates of $\delta$ under $\langle U, V\rangle$ may be labelled as $\delta_{i}$, where $1 \leq i \leq n$. Relation (v) states that $\delta_{2}=\delta_{1} \Delta^{-(q+1)}$; so, using (iv), $\delta_{1}$ commutes with $\delta_{2}$, and hence $\delta_{i}$ commutes with $\delta_{j}$ for all $i, j$. By (iv), $\delta$ commutes with $\Delta_{12}$ and with $\Delta_{23}$, and hence $\delta_{i}$ commutes with $\Delta_{j k}$ for all $i$ and for all $j \neq k$. It follows from (v) that $\delta_{i} \delta_{j}^{-1}=\Delta_{i j}^{q+1}$ for all $i \neq j$. Thus if $A$ is the subgroup of $G$ generated by $\left\{\delta_{i} \mid i>0\right\} \cup\left\{\Delta_{i j} \mid i \neq j\right\}$ and $B=\left\langle\left\{\Delta_{i j}\right\}\right\rangle$, then $A$ is an abelian group, and $A / B$ is generated by $\left\{\delta_{i} \mid i>0\right\}$ subject to the relations $\delta_{i}=\delta_{j}$, and $\delta_{1}^{q-1}=1$. So the group presented has order at most $(q-1)\left(q^{2}-1\right)^{n-1} n$ !. But the corresponding matrix group has this order, so the theorem is proved when $n>2$. The case $n=2$ is proved similarly.

Let $N$ be the subgroup of $\mathrm{SU}(2 n, q)$ generated by $\{Z, \delta, \Delta, U, V\}$, omitting $V$ if $n=2$. It has order $(q-1)\left(q^{2}-1\right)^{n-1} 2^{n} n$ !.

Theorem 7.3. Let $G$ be the group generated by $\{Z, \delta, \Delta, U, V\}$, omitting $V$ if $n=2$, subject to the relations listed below. Then $G$ is isomorphic to $N$.

(i) Defining relations for $\langle\delta, \Delta, U, V\rangle$ as in Theorem 7.2 .

(ii) $Z^{2}=\delta^{(q-1) / 2}$ if $q$ is odd; $Z^{2}=1$ if $q$ is even.

(iii) $\left[Z, U^{V}\right]=1$, and if $n>3$ then $[Z, V U]=1$.

(iv) $\left[Z, Z^{U}\right]=1$.

(v) $\delta=\left[\Delta^{-1}, Z\right]$

(vi) $\left[Z, \Delta^{V}\right]=1$.

(vii) $\delta^{Z}=\delta^{-1}$.

(viii) $\left[\delta, Z^{U}\right]=1$ if $n=2$.

We omit those relations that involve $V$ if $n=2$.

Proof. Suppose first that $n>2$. As with the previous theorem, relations (iii) imply that we have elements $Z_{i}$ in $G$ that are permuted by $\langle U, V\rangle$ via the natural action of $S_{n}$ on the suffices. Relation (iv) asserts that $\left[Z_{1}, Z_{2}\right]=1$; so the group generated by the $Z_{i}$ is abelian. Relation (vi) states that $\Delta_{23}$ is centralised by $Z_{1}$; so $\Delta_{i j}$ is centralised by $Z_{k}$ for all $i \neq k \neq j$. It follows from $(\mathrm{v})$ that $\delta_{i}=\left[\Delta_{i j}^{-1}, Z_{i}\right]$ for all $j \neq i$, and hence $\delta_{i}$ is centralised by $Z_{k}$ for all $k \neq i$ since $n>2$. It now follows, from (vii), that the group $A$ in the proof of the previous theorem is normalised by $Z_{1}$, and hence by $Z_{i}$ for all $i$. By (ii), $A$ contains $Z_{1}^{2}$, and hence contains $Z_{i}^{2}$ for all $i$. Thus $G$ has at most the stated order; since the corresponding matrix group has this order the theorem is proved for $n>2$. 
The proof for $n=2$ is similar, except for the proof that $\delta_{i}$ commutes with $Z_{j}$ for all $i \neq j$. This follows from (viii), which states that $\delta_{1}$ commutes with $Z_{2}$ if $n=2$.

Theorem 7.4. The subgroup of $\mathrm{SU}(2 n, q)$ generated by $\{\tau, \delta, Z\}$ is isomorphic to $\operatorname{SL}(2, q)$, and the subgroup $H$ generated by $\left\{\sigma, \Delta, U Z^{2}\right\}$ is isomorphic to $\mathrm{SL}\left(2, q^{2}\right)$.

Proof. The first statement is obvious. Observe that $H$ acts on $\left\langle e_{1}, e_{2}\right\rangle$ as $\operatorname{SL}\left(2, q^{2}\right)$, and similarly on $\left\langle f_{1}, f_{2}\right\rangle$, the actions corresponding under the inverse transpose automorphism followed by the Frobenius automorphism.

Theorem 7.5. The centraliser $H$ of $\sigma=\sigma_{12}(1)$ in $N$ is generated by the following elements:

(i) $U^{V^{2}}$ if $n>3$, and $V U U^{V}$ if $n>4$;

(ii) $\Delta^{V^{2}}$ if $n>3$ and $q$ is odd, and $\delta^{V^{2}}$ if $n=3$ and $q$ is odd;

(iii) $Z^{V^{2}}$ if $n>2$;

(iv) $\Delta \Delta^{2 V}$ if $n>2$;

(v) if $n=2$ then $\delta \delta^{U}$ if $q$ is even, and $\Delta^{(q+1) / 2} \delta^{-1}$ if $q$ is odd;

(vi) $Z U Z^{-1}$.

If $n>2$ then $H$ has order $(q-1)\left(q^{2}-1\right)^{n-2} 2^{n-1}(n-2)$ !; if $n=2$ then $H$ has order $4(q-1)$ if $q$ is odd, and order $2(q-1)$ if $q$ is even.

Proof. Let $n>2$. The orbit of $\sigma$ under conjugation by $N$ is $\left\{\sigma_{i j}(\alpha)|1 \leq| i|\neq| j \mid \leq n, \alpha \in\right.$ $\left.\operatorname{GF}\left(q^{2}\right)^{\times}\right\}$. But the $4 n(n-1)\left(q^{2}-1\right)$ matrices defined are equal in pairs, as shown in Section 7.1. The orbit has size $2 n(n-1)\left(q^{2}-1\right)$, so $H$ has order $(q-1)\left(q^{2}-1\right)^{n-2} 2^{n-1}(n-2)$ !. Let $K$ be the group generated by the itemised elements. Clearly $K$ is a subgroup of $H$. The subgroup $K_{0}$ of $K$ generated by the elements in (i) is the copy of $S_{n-2}$ that permutes the basis vectors $\left\{e_{1}, e_{2}, \ldots, e_{n}\right\}$, fixing $e_{1}$ and $e_{2}$, and also permutes $\left\{f_{1}, \ldots, f_{n}\right\}$, fixing $f_{1}$ and $f_{2}$.

Let $n>2$ and $q$ be even. Observe that

$$
\Delta \Delta^{2 V}=\operatorname{diag}\left(\omega^{-1}, \omega^{q}, \omega^{-1}, \omega^{q}, \omega^{2}, \omega^{-2 q}, 1,1, \ldots, 1\right) .
$$

Its $K_{0}$-conjugates generate an abelian group $A$ of order $\left(q^{2}-1\right)^{n-2}$. Define

$$
b:=\left[\Delta \Delta^{2 V}, Z^{V^{2}}\right]=\operatorname{diag}\left(1,1,1,1, \omega_{0}^{-2}, \omega_{0}^{2}, 1,1, \ldots, 1\right) .
$$

Clearly $B:=\langle A, b\rangle$ is a diagonal subgroup of $K$ of order $(q-1)\left(q^{2}-1\right)^{n-2}$ that is normalised by $K_{0}$. Also, $Z U Z^{-1}$ and the $K_{0}$-conjugates of $Z^{V^{2}}$ generate an abelian group $C$ of order $2^{n-1}$ that intersects $B$ in the identity. Since $\left\langle K_{0}, B, C\right\rangle$ has the correct order, it is the centraliser of $\sigma$.

Let $n>3$ and $q$ be odd. Observe that

$$
\Delta^{V^{2}}=\operatorname{diag}\left(1,1,1,1, \omega^{-1}, \omega^{q}, \omega, \omega^{-q}, 1,1, \ldots, 1\right) .
$$

Its $\left\langle K_{0}, Z^{V^{2}}\right\rangle$-conjugates generates the group

$$
\left\{\operatorname{diag}\left(1,1,1,1, \alpha_{3}, \alpha_{3}^{-q}, \ldots, \alpha_{n}, \alpha_{n}^{-q}\right): \prod_{i} \alpha_{i} \in \mathrm{GF}(q)^{\times}\right\},
$$

which has order $\left(q^{2}-1\right)^{n-3}(q-1)$. This group and $\Delta \Delta^{2 V}$ generate a group $D$ of order $\left(q^{2}-1\right)^{n-2}(q-1)$, which is normalised by the group $C=\left\langle K_{0}, Z^{V^{2}}, Z U Z^{-1}\right\rangle$. Now $C$ 
has order $2^{2 n-3}(n-2)$ !, and intersects $D$ in a group of order $2^{n-2}$, so $C D$ has order $(q-1)\left(q^{2}-1\right)^{n-2} 2^{n-1}(n-2)$ ! and so is the centraliser of $\sigma$ in $N$.

Let $n=3$ and $q$ be odd. Now $D=\left\langle\Delta \Delta^{2 V}, \delta^{V^{2}}\right\rangle$ has order $(q-1)\left(q^{2}-1\right)$, and is normalised by $C=\left\langle Z^{V^{2}}, Z U Z^{-1}\right\rangle$, which has order 8 , and $C \cap D$ has order 2, so $C D$ has order $4(q-1)\left(q^{2}-1\right)$, as required.

Finally let $n=2$. The orbit of $\sigma$ under conjugation by $N$ is $\left\{\sigma_{ \pm 1, \pm 2}\left(\alpha^{2}\right) \mid \alpha \in \operatorname{GF}\left(q^{2}\right)^{\times}\right\}$, and hence has size $4\left(q^{2}-1\right)$ in $N$ if $q$ is even, and $2\left(q^{2}-1\right)$ if $q$ is odd. So the centraliser of $\sigma$ has order $2(q-1)$ if $q$ is even, and order $4(q-1)$ if $q$ is odd. Items (v) and (vi) generate a copy of $D_{2(q-1)}$ if $q$ is even, else a copy of $S D_{4(q-1)}$; and these groups have the required order.

Theorem 7.6. The centraliser of $\tau=\tau_{1}(1)$ in $N$ has order $\left(q^{2}-1\right)^{n-1} 2^{n-1}(n-1)$ ! and is generated by the following elements:

(i) $U^{V}$ if $n>2$, and $V U$ if $n>3$;

(ii) if $n=2$ and $q$ is odd then $\delta^{U}$, and if $n>2$ then $\Delta^{V}$;

(iii) $Z^{U}$;

(iv) $\Delta^{2} \delta^{-1}$.

Proof. Recall that the order of $N$ is $(q-1)\left(q^{2}-1\right)^{n-1} 2^{n} n$ !. The orbit of $\tau$ under conjugation by $N$ is $\left\{\tau_{ \pm i}(s) \mid 1 \leq i \leq n, s \in \mathrm{GF}(q)^{\times}\right\}$, as $\tau_{i}(s)^{\Delta}=\tau_{i}\left(s \omega_{0}\right)$, and has size $2(q-1) n$.

The elements in (i) imply that $\tau$ is centralised by the copy of $S_{n-1}$ in $\langle U, V\rangle$ that fixes the basis vectors $e_{1}$ and $f_{1}$.

Suppose that $n>2$ and $q$ is odd. Now $\Delta^{V}=\operatorname{diag}\left(1,1, \omega^{-1}, \omega^{q}, \omega, \omega^{-q}, 1, \ldots, 1\right)$, together with its conjugates under this copy of $S_{n-1}$, generates a group $A$ that is the direct product of $n-2$ cyclic groups of order $q^{2}-1$. This group intersects the cyclic group of order $\left(q^{2}-1\right) / 2$ generated by $\Delta^{2} \delta^{-1}=\operatorname{diag}\left(\omega^{q-1}, \omega^{q-1}, \omega^{2}, \omega^{-2 q}, 1, \ldots, 1\right)$ in the identity, so this element and $A$ generate a group $B$ of order $\left(q^{2}-1\right)^{n-1} / 2$. Define $g:=\left(\Delta^{V}\right)^{Z^{U}}=\Delta^{V} \times \operatorname{diag}\left(1,1, \omega_{0}, \omega_{0}^{-1}, 1, \ldots, 1\right)$. Now $g^{2}=\Delta^{2 V}\left(\Delta^{2} \delta^{-1}\right)^{q+1}$ lies in $B$, but $g$ does not, and $C:=\langle B, g\rangle$ has order $\left(q^{2}-1\right)^{n-1}$. Thus the group generated by the listed elements contains an abelian subgroup $C$ of order $\left(q^{2}-1\right)^{n-1}$, extended by an elementary abelian group of order $2^{n-1}$ generated, modulo $C$, by $\left\{Z_{i} \mid i>1\right\}$, extended by a copy of $S_{n-1}$. This group has index $2(q-1) n$ in $N$, and so is the centraliser of $\tau$.

If $n>2$ and $q$ is even, then the proof is simpler: $\Delta^{2} \delta^{-1}$ and the $S_{n-1}$-conjugates of $\Delta^{V}$ now generate a group of order $\left(q^{2}-1\right)^{n-1}$.

The proof is similar for $n=2$. We need to prove that the given elements generate a group that contains a diagonal subgroup of order $q^{2}-1$. If $q$ is even, then $\Delta^{2} \delta^{-1}$ generates such a subgroup. If $q$ is odd, then this element has order $\left(q^{2}-1\right) / 2$, and $\delta^{U}=\operatorname{diag}\left(1,1, \omega_{0}^{-1}, \omega_{0}\right)$ does not lie in $\left\langle\Delta^{2} \delta^{-1}\right\rangle$, but its square, which is $\left(\Delta^{2} \delta^{-1}\right)^{-(q+1)}$, does.

Theorem 7.7. Let $q=p^{e}$ for a prime $p$ and let $n \geq 2$. Let $G$ be the group generated by $\{\sigma, \tau, Z, \delta, \Delta, U, V\}$, omitting $V$ if $n=2$, subject to the relations given below. Then $G$ is isomorphic to $\mathrm{SU}(2 n, q)$.

(i) Defining relations for $N=\langle Z, \delta, \Delta, U, V\rangle$ as in Theorem 7.3 , omitting $V$ if $n=2$, and omitting relations (iii) and (vi) of Theorem $\mathbf{7 . 1}$, relation (ii) of Theorem $\mathbf{7 . 2}$, and relations (ii) and (vii) of Theorem 7.3 .

(ii) Relations that state that the elements listed in Theorem 7.5 centralise $\sigma$. 
(iii) Relations that state that the elements listed in Theorem 7.6 centralise $\tau$, but omitting the relation $[\tau, V U]=1$.

(iv) Relations that present $\mathrm{SL}(2, q)$ on $\{\tau, \delta, Z\}$, and $\mathrm{SL}\left(2, q^{2}\right)$ on $\left\{\sigma, \Delta, U Z^{2}\right\}$ as in Theorem 7.4 .

(v) The following instances of Steinberg relations:

(a) $[\sigma, \tau]=1$;

(b) $\left[\sigma, \sigma^{Z}\right]=\tau^{2 Z U}$, and $\left[\sigma^{\Delta}, \sigma^{Z}\right]=\tau^{Z U \Delta^{m}}$, where $\omega_{0}^{m}=\omega^{2}+\omega^{2 q}$; the RHS of the first relation may be replaced by 1 if $q$ is even, and the RHS of the second relation must be replaced by 1 if $q=3$;

(c) $\left[\sigma, \tau^{Z}\right]=\sigma^{Z} \tau^{-Z U}$;

(d) if $n>2$ then $\left[\sigma, \sigma^{U^{V}}\right]=1$, and if $n=3$ and $q=2$ then $\left[\sigma, \sigma^{U^{V} \Delta}\right]=1$;

(e) if $n>2$ then $\left[\sigma, \sigma^{V}\right]=\sigma^{V U}$, and if $n=3$ and $q=2$ then $\left[\sigma, \sigma^{V \Delta}\right]=\sigma^{V U \Delta^{-1}}$;

(f) if $n<4$ then $\left[\tau, \tau^{U}\right]=1$;

(g) if $n=3$ then $\left[\tau, \sigma^{V}\right]=1$;

(h) if $n>3$ then $\left[\sigma, \sigma^{V^{2}}\right]=1$.

(vi) If $n=2$ and $q=3$ then $\tau=\left[\sigma^{-U Z}, \sigma\right]^{\Delta}$.

Proof. The relations omitted from (i) are supplied by (iv). In odd characteristic the relation $\Delta^{U}=\Delta^{-1}$, which is (iii) of Theorem [7.1, follows from the relation $\Delta^{U Z^{2}}=\Delta^{-1}$ implied by (iv) of Theorem [7.4, since $Z^{2}=\delta^{(q-1) / 2}$ by the same relations, and $[\delta, \Delta]=1$ by (iv) of Theorem 7.2 .

The relation $[\tau, V U]=1$ omitted from (iii) may be supplied using relation (v)(b) exactly as with the presentation for $\operatorname{Sp}(2 n, q)$; here the argument applies in all characteristics.

As to (v)(b), note that $\omega^{2}+\omega^{2 q}=0$ if $q=3$.

Relations (i), (ii) and (iii), together with (iv) if $n=2$, allow us to define elements $\sigma_{i j}(\alpha)$ and $\tau_{i}(s)$ of $G$ that are permuted by $N$.

The following Steinberg relations must now be proved to hold in $G$. Here $i, j, k, l$ are distinct positive integers in the range $[1, n]$, and $\alpha, \beta \in \mathrm{GF}\left(q^{2}\right)$, and $s, t \in \operatorname{GF}(q)$.

(1) $\sigma_{i j}(\alpha)=\sigma_{-j,-i}\left(-\alpha^{q}\right) ; \sigma_{i,-j}(\alpha)=\sigma_{j,-i}\left(\alpha^{q}\right) ; \sigma_{-i, j}(\alpha)=\sigma_{-j, i}\left(\alpha^{q}\right)$;

(2) $\tau_{i}(s) \tau_{i}(t)=\tau_{i}(s+t), \tau_{i}(s)^{p}=1$

(3) $\sigma_{i j}(\alpha) \sigma_{i j}(\beta)=\sigma_{i j}(\alpha+\beta), \sigma_{i j}(\alpha)^{p}=1$;

(4) $\left[\sigma_{i j}(\alpha), \tau_{i}(s)\right]=\left[\sigma_{i j}(\alpha), \tau_{-j}(s)\right]=1$;

(5) $\left[\sigma_{i j}(\alpha), \sigma_{-i, j}(\beta)\right]=\tau_{-j}\left(\alpha^{q} \beta+\alpha \beta^{q}\right)$;

(6) $\left[\sigma_{i j}(\alpha), \sigma_{i,-j}(\beta)\right]=\tau_{i}\left(\alpha^{q} \beta+\alpha \beta^{q}\right)$;

(7) $\left[\sigma_{i j}(\alpha), \tau_{-i}(s)\right]=\sigma_{-i, j}(\alpha s) \tau_{-j}\left(-\alpha^{q+1} s\right)$,

$\left[\sigma_{i j}(\alpha), \tau_{j}(s)\right]=\sigma_{j,-i}\left(\alpha^{q} s\right) \tau_{i}\left(-\alpha^{q+1} s\right)$

(8) $\left[\sigma_{i j}(\alpha), \sigma_{i k}(\beta)\right]=1$ if $n>2$;

(9) $\left[\sigma_{i j}(\alpha), \sigma_{k j}(\beta)\right]=1$ if $n>2$;

(10) $\left[\sigma_{i j}(\alpha), \sigma_{j k}(\beta)\right]=\sigma_{i k}(\alpha \beta)$ if $n>2$;

(11) $\left[\sigma_{i j}(\alpha), \sigma_{-i, k}(\beta)\right]=\sigma_{-j, k}\left(-\alpha^{q} \beta\right)$,

$\left[\sigma_{i j}(\alpha), \sigma_{k,-j}(\beta)\right]=\sigma_{i,-k}\left(\alpha \beta^{q}\right)$,

$\left[\sigma_{i j}(\alpha), \sigma_{k,-i}(\beta)\right]=\left[\sigma_{i j}(\alpha), \sigma_{-j, k}(\beta)\right]=1$, where in all cases $n>2$;

(12) $\left[\tau_{i}(s), \tau_{j}(t)\right]=\left[\sigma_{i j}(\alpha), \tau_{k}(s)\right]=\left[\sigma_{i j}(\alpha), \sigma_{k l}(\beta)\right]=1$ for all $n$, if $n>2$, and if $n>3$ respectively. 
Similar relations hold when we lift the restriction that $i, j, k$ and $l$ are positive. These relations are obtained from those listed by conjugating with $Z_{i}, Z_{j}, Z_{k}$, and $Z_{l}$, as appropriate, and may differ from them in the signs of the coefficient: $\sigma_{-i, j}(\alpha)^{Z_{i}}=\sigma_{i, j}(-\alpha)$ for $i>0$ and $j$ positive or negative, and similarly $\sigma_{i,-j}(\alpha)^{Z_{j}}=\sigma_{i, j}(-\alpha)$ for $j>0$ and $i$ positive or negative. It suffices to prove that these relations hold in $G$ when the suffices $i$ to $l$ are positive. We assume that $(i, j, k, l)=(1,2,3,4)$; the general case follows by conjugation in $\langle U, V\rangle$.

Relation (vi) is also required, since the Steinberg relations define the universal covering group of the simple group, and this is not the classical group in the case of $\operatorname{PSU}(2 n, q)$ when $(n, q)$ is $(2,2)$, or $(2,3)$, or $(3,2)$. Our presentations include relations that are not Steinberg relations, in particular those for $N$. We find, by coset enumeration, that our presentations in these three cases are correct; in particular, no further relation is needed if $(n, q)$ is $(2,2)$ or $(3,2)$. Note that $\mathrm{SU}(4,2)$ is simple, and its universal covering group is isomorphic to $\operatorname{Sp}(4,3)$, which has centre of order 2 .

Relations (1) hold by definition, using (i) and (iii), but $Z_{i}^{2}$ commutes with $\tau_{ \pm i}(s)$ and conjugates $\sigma_{ \pm i, \pm j}(\alpha)$ to its inverse.

Relations (2) and (3) follow from Theorem 7.4 and relations (i) to (iv).

We now prove the first relation in (4). The instance $i=1, j=2, \alpha=s=1$ is given by relation (a). Let $P$ be the set of pairs $(\alpha, s)$ for which (4) holds with $i=1$ and $j=2$. Clearly if $(\alpha, s) \in P$ and $(\beta, s) \in P$, then $(\alpha+\beta, s) \in P$; and if $(\alpha, s) \in P$ and $(\alpha, t) \in P$, then $(\alpha, s+t) \in P$. Conjugating by $\Delta$ shows that if $(\alpha, s) \in P$ then $\left(\omega^{2} \alpha, \omega_{0} s\right) \in P$, and so $\left(\omega^{2(q-1)}, 1\right) \in P$. If $q \neq 3$ then $\omega^{2(q-1)}$ generates $\operatorname{GF}\left(q^{2}\right)$ as a field, so $(\alpha, 1) \in P$ for all $\alpha \in \operatorname{GF}\left(q^{2}\right)$. Since $\omega_{0}$ generates $\operatorname{GF}(q)$ as a field, it follows that $(\alpha, s) \in P$ for all $\alpha$ in $\operatorname{GF}\left(q^{2}\right)$ and all $s \in \operatorname{GF}(q)$. For $q=3$ (or more generally prime), if $(\alpha, s) \in P$ for one $s$ in $\operatorname{GF}(q)^{\times}$then $(\alpha, s) \in P$ for all $s$ in $\operatorname{GF}(q)$, and since $\omega^{2}$ generates $\operatorname{GF}\left(q^{2}\right)$ as a field, again $(\alpha, s) \in P$ for all $\alpha$ in $\operatorname{GF}\left(q^{2}\right)$, and all $s \in \operatorname{GF}(q)$. The second relation in (4) follows from the first by applying (1), and conjugating by $Z_{i} Z_{j}$.

We now prove relation (5). Let $P$ be the set of pairs $(\alpha, \beta)$ such that (5) holds with $i=1$ and $j=2$. Relation (b) states that $(1,1) \in P$ and $\left(\omega^{2}, 1\right) \in P$. Relations (2) and (4) show that if $\left(\alpha, \beta_{1}\right)$ and $\left(\alpha, \beta_{2}\right)$ lie in $P$, then so does $\left(\alpha, \beta_{1}+\beta_{2}\right)$. Similarly, if $\left(\alpha_{1}, \beta\right)$ and $\left(\alpha_{2}, \beta\right)$ lie in $P$, then so does $\left(\alpha_{1}+\alpha_{2}, \beta\right)$. Conjugating by $\Delta$ shows that if $(\alpha, \beta) \in P$ then $\left(\omega^{2} \alpha, \omega^{1-q} \beta\right) \in P$. If $q$ is odd then $\left(\omega^{q+1} \alpha,-\beta\right)=\left(\omega_{0} \alpha,-\beta\right) \in P$, so $\left(\omega_{0} \alpha, \beta\right) \in P$. Similarly, if $q$ is even, then $\left(\omega_{0}^{2} \alpha, \beta\right) \in P$, and hence $\left(\omega_{0} \alpha, \beta\right) \in P$. Conjugating by $\Delta_{21}$ shows that if $(\alpha, \beta) \in P$ then $\left(\omega^{-2} \alpha, \omega^{q-1} \beta\right) \in P$ and so $\left(\alpha, \omega_{0} \beta\right) \in P$. It follows that $P=\mathrm{GF}\left(q^{2}\right) \times \mathrm{GF}\left(q^{2}\right)$ since $\omega^{2} \notin \mathrm{GF}(q)$.

We now prove relation (6). By (5), $\left[\sigma_{i j}(\alpha), \sigma_{-i, j}(\beta)\right]=\tau_{-j}\left(\alpha^{q} \beta+\alpha \beta^{q}\right)$. Conjugating by $Z_{j}$ now gives $\left[\sigma_{i,-j}(\alpha), \sigma_{-i,-j}(\beta)\right]=\tau_{j}\left(\alpha^{q} \beta+\alpha \beta^{q}\right)$. Equivalently $\left[\sigma_{j,-i}(\alpha), \sigma_{-j,-i}(\beta)\right]=$ $\tau_{i}\left(\alpha^{q} \beta+\alpha \beta^{q}\right)$. By (1) this can be written as $\left[\sigma_{i,-j}\left(\alpha^{q}\right), \sigma_{i, j}\left(-\beta^{q}\right)\right]=\tau_{i}\left(\alpha^{q} \beta+\alpha \beta^{q}\right)$, which is equivalent to $(6)$.

We now prove the first relation in (7). An instance with $i=1, j=2$, and $\alpha=s=1$ is given by relation (c). Let $P$ be the set of pairs $\left\{(\alpha, s) \mid \alpha \in \mathrm{GF}\left(q^{2}\right), s \in \mathrm{GF}(q)\right\}$ for which 
(7) holds with $(i, j)=(1,2)$. Suppose that $(\alpha, s)$ and $(\beta, s)$ lie in $P$. Now

$$
\begin{aligned}
{\left[\sigma_{12}(\alpha+\beta), \tau_{-1}(s)\right] } & =\left[\sigma_{12}(\alpha), \tau_{-1}(s)\right]^{\sigma_{12}(\beta)}\left[\sigma_{12}(\beta), \tau_{-1}(s)\right] \\
& =\left(\sigma_{-1,2}(\alpha s) \tau_{-2}\left(-\alpha^{q+1} s\right)\right)^{\sigma_{12}(\beta)} \sigma_{-1,2}(\beta s) \tau_{-2}\left(-\beta^{q+1} s\right) \\
& =\sigma_{-1,2}(\alpha s) \tau_{-2}\left(-\alpha^{q} \beta s-\alpha \beta^{q} s\right) \tau_{-2}\left(-\alpha^{q+1} s\right) \sigma_{-1,2}(\beta s) \tau_{-2}\left(-\beta^{q+1} s\right) \\
& =\sigma_{-1,2}((\alpha+\beta) s) \tau_{-2}\left(-(\alpha+\beta)^{q+1} s\right) .
\end{aligned}
$$

Here we used relations (2)-(5). So if $(\alpha, s)$ and $(\beta, s)$ lie in $P$ then so does $(\alpha+\beta, s)$. Similarly, using (2), (3), (4), and (12), one proves that, if $(\alpha, s)$ and $(\alpha, t)$ lie in $P$ then so does $(\alpha, s+t)$. Conjugating by $\Delta$ shows that if $(\alpha, s) \in P$ then $\left(\alpha \omega^{2}, s \omega_{0}\right) \in P$, so $\left(\alpha \omega^{2(q-1)}, s\right) \in P$. As in the proof of (4), it follows that if $(\alpha, s)$ lies in $P$ for one value of $\alpha$ then this holds for all $\alpha$; and hence $(\alpha, s) \in P$ for all $\alpha$ and all $s$. The second relation in (7) follows from the first by applying (1), and conjugating by $Z_{i} Z_{j}$.

We now prove relation (8). Clearly if this holds for $\left(\alpha_{1}, \beta\right)$ and $\left(\alpha_{2}, \beta\right)$ then it also holds for $\left(\alpha_{1}+\alpha_{2}, \beta\right)$, and similarly with the roles of $\alpha$ and $\beta$ reversed. Let $P$ be the set of all pairs $(\alpha, \beta)$ for which (8) holds. If $(\alpha, \beta) \in P$, then conjugating by $\Delta_{i j}$ and $\Delta_{i k}$ shows that both $\left(\omega^{2} \alpha, \omega \beta\right) \in P$ and $\left(\omega \alpha, \omega^{2} \beta\right) \in P$, so $\left(\alpha, \omega^{-3} \beta\right) \in P$. If $q>2$ then $\omega^{3}$ generates $\mathrm{GF}\left(q^{2}\right)$ as a field, so (8) follows from one instance, such as (d). If $q=2$ and $n>3$ then the general case also follows from one instance, by conjugation with $\Delta_{j l}$ and $\Delta_{k l}$. If $(n, q)=(3,2)$ then we need the additional relation in $(\mathrm{d})$, which asserts that $(1, \omega) \in P$.

Now (9) follows from (8) since $\sigma_{i j}(\alpha)=\sigma_{-j,-i}\left(-\alpha^{q}\right)$.

Using (8) and (9) it follows that if (10) holds for $\left(\alpha_{1}, \beta\right)$ and $\left(\alpha_{2}, \beta\right)$ then it holds for $\left(\alpha_{1}+\alpha_{2}, \beta\right)$, and similarly with $\alpha$ and $\beta$ reversed. The result now follows from (e) exactly as in the proof of $(8)$, and again we need an extra relation if $(n, q)=(3,2)$, which here gives $\left[\sigma_{12}(2), \sigma_{23}(\omega)\right]=\sigma_{13}(\omega)$.

The relations in (11) follow from (8)-(10) by conjugating with $Z_{i}, Z_{j}$ and $Z_{k}$, as appropriate, and using (1).

If $n>3$ then relations (f) and (g) may be supplied using (h) and (b). Relations (12) now follow.

It follows that the conjugates of $\sigma$ and $\tau$ under $N=\langle Z, \Delta, U, V\rangle$, omitting $V$ if $n=2$, generate a subgroup $H$ of $G$ that is isomorphic to $\mathrm{SU}(2 n, q)$. But $Z$ and $\Delta$ and $U$ lie in $H$, as do their conjugates under $N$, and hence if $n>2$ then $V$ lies in $H$, as a product of $N$-conjugates of $U$. Thus $G=H$, as required.

We shall need the following result.

Corollary 7.8. If $q$ is odd, then a presentation for $\mathrm{SU}(4, q) /\left\langle-I_{4}\right\rangle$ is obtained from the presentation for $\mathrm{SU}(4, q)$ of Theorem 7.7 by replacing the presentation for $\mathrm{SL}\left(2, q^{2}\right)$ on $\left\{\sigma, \Delta, U Z^{2}\right\}$ by a presentation of $\operatorname{PSL}\left(2, q^{2}\right)$ on these generators as in Theorem 3.1 .

7.3. A presentation for $\operatorname{PSU}(2 n, q)$. The centre of $\mathrm{SU}(2 n, q)$ has order $\operatorname{gcd}(q+1,2 n)$.

Lemma 7.9. Let $v_{2}(x)$ denote the 2 -adic value of the integer $x$. If $v_{2}(n) \geq v_{2}(q+1)$ then the centre of $\mathrm{SU}(2 n, q)$ is generated by $\left(\Delta^{q-1} U V^{-1}\right)^{(n-1)(q+1) / \operatorname{gcd}(q+1, n)}$. If $v_{2}(q+1)>$ $v_{2}(n)$ then the centre is generated by $Z^{2}\left(\Delta^{q-1} U V^{-1}\right)^{(n-1)(q+1) /(2 \operatorname{gcd}(q+1, n))}$. 
Proof. Let $\theta:=\omega^{1-q}$, so $\Delta^{q-1}=\operatorname{diag}\left(\theta, \theta, \theta^{-1}, \theta^{-1}, 1,1, \ldots, 1\right)$. If $g:=\left(\Delta^{q-1} U V^{-1}\right)^{n-1}$ then $g=\operatorname{diag}\left(\theta^{(n-1)}, \theta^{(n-1)}, \theta^{-1}, \theta^{-1}, \ldots, \theta^{-1}\right)$. So $g^{k}$ is a scalar matrix if and only if $\theta^{n k}=$ 1 ; equivalently, $k$ is a multiple of $(q+1) / \operatorname{gcd}(q+1, n)$. So $g^{(q+1) / \operatorname{gcd}(q+1, n)}$ is a scalar element of $\mathrm{SU}(2 n, q)$ of order $\operatorname{gcd}(q+1, n)$. If $v_{2}(n) \geq v_{2}(q+1)$ then $\operatorname{gcd}(q+1, n)=\operatorname{gcd}(q+1,2 n)$, as required. Now suppose that $v_{2}(q+1)>v_{2}(n)$. Observe that $Z^{2} g^{(q+1) /(2 \operatorname{gcd}(q+1, n))}$ has eigenvalues $-\theta^{(n-1)(q+1) /(2 \operatorname{gcd}(q+1, n)}$ and $\theta^{-(q+1) /(2 \operatorname{gcd}(q+1, n))}$, and these are equal, so we have a scalar element of order $2 \operatorname{gcd}(q+1, n)=\operatorname{gcd}(q+1,2 n)$, as required.

7.4. Standard generators for $\mathrm{SU}(2 n, q)$. In Table 1 of [10, 21 the standard generators for $\mathrm{SU}(2 n, q)$ are labelled $s, t, \delta, u, v, x, y$. Observe that $s=Z^{-1} ; t=\tau^{-1} ; u=U ; v=$ $V ; x=\sigma ; y=\Delta^{-1}$; and the standard generator $\delta$ is the inverse of the presentation generator $\delta$.

\section{A presentation for $\mathrm{SU}(2 n+1, q)$ FOR $n>1$}

8.1. Generators and notation. Let $q=p^{e}$ for a prime $p$. Let $\omega$ be a primitive element of $\operatorname{GF}\left(q^{2}\right)$, and let $\omega_{0}=\omega^{q+1}$, so $\omega_{0}$ is a primitive element of $\operatorname{GF}(q)$. Define $\psi=\omega^{(q+1) / 2}$ if $q$ is odd, and $\psi=1$ if $q$ is even. Let $\phi$ be a fixed element of $\operatorname{GF}\left(q^{2}\right)$ of trace -1 : define $\phi=-1 / 2$ if $q$ is odd, and $\phi=\omega /\left(\omega+\omega^{q}\right)$ if $q$ is even.

Let $n>1$. We take a basis $\left(e_{1}, f_{1}, e_{2}, f_{2}, \ldots, e_{n}, f_{n}, w\right)$ of the natural module, with symmetric bilinear form defined by $e_{i} \cdot f_{i}=f_{i} \cdot e_{i}=1$ for all $i$, and $w \cdot w=1$, and the form vanishes on all other pairs of basis vectors. So $\mathrm{SU}(2 n+1, q)$ contains $\mathrm{SU}(2 n, q)$ as defined in Section 7.1. We retain the notation of that section and define the following new elements of $\mathrm{SU}(2 n+1, q)$, where $1 \leq i \leq n$ and $\alpha \in \mathrm{GF}\left(q^{2}\right)$.

$$
\begin{aligned}
& t_{i}=\left(e_{i} \mapsto f_{i}, f_{i} \mapsto e_{i}, w \mapsto-w\right) ; \\
& \Gamma_{i}=\left(e_{i} \mapsto \omega^{-1} e_{i}, f_{i} \mapsto \omega^{q} f_{i}, w \mapsto \omega^{1-q} w\right) ; \\
& \nu_{i}(\alpha)=\left(e_{i} \mapsto e_{i}+\phi \alpha^{q+1} f_{i}+\alpha w, w \mapsto w-\alpha^{q} f_{i}\right) ; \\
& \nu_{i}(\alpha)^{-1}=\left(e_{i} \mapsto e_{i}-(\phi+1) \alpha^{q+1} f_{i}-\alpha w, w \mapsto w+\alpha^{q} f_{i}\right) ; \\
& \nu_{-i}(\alpha)=\left(f_{i} \mapsto f_{i}+\phi \alpha^{q+1} e_{i}-\alpha w, w \mapsto w+\alpha^{q} e_{i}\right)=\nu_{i}(\alpha)^{t_{i}} .
\end{aligned}
$$

Recall that $\sigma_{i j}(\alpha)=\left(e_{i} \mapsto e_{i}+\alpha e_{j}, f_{j} \mapsto f_{j}-\alpha^{q} f_{i}\right)$ and $\tau_{i}(s)=\left(e_{i} \mapsto e_{i}-s \psi f_{i}\right)$, where $1 \leq i, j \leq n$ and $i \neq j$, and $s \in \mathrm{GF}(q)$.

All these elements of $\mathrm{SU}(2 n+1, q)$ centralise those basis elements that they are not stated to move.

8.2. A presentation for $\mathrm{SU}(2 n+1, q)$. We give a presentation for $\mathrm{SU}(2 n+1, q)$ on the generating set $\left\{\nu=\nu_{1}(1), \sigma=\sigma_{12}(1), \tau=\tau_{1}(1), \Gamma=\Gamma_{1}, t=t_{1}, U, V\right\}$, omitting $V$ if $n=2$.

The remaining generators used in the presentation for $\mathrm{SU}(2 n, q)$ are defined as follows: $\Delta=\Gamma \Gamma^{-U}$; if $q$ is even, then $Z=t$, else $Z=t \Gamma^{(q+1) / 2}$; and $\delta=\Delta \Delta^{-Z}=\Gamma^{q+1}$.

Let $N$ be the subgroup of $\mathrm{SU}(2 n+1, q)$ generated by $\{\Gamma, t, U, V\}$, omitting $V$ if $n=2$. It is isomorphic to $\left(C_{q^{2}-1}: C_{2}\right) \backslash S_{n}$, where the 2-cycle generated by $t$ acting on the $\left(q^{2}-1\right)$-cycle generated by $\Gamma$ acts as $g \mapsto g^{-q}$.

Theorem 8.1. Let $G$ be the group generated by $\{\Gamma, t, U, V\}$, omitting $V$ if $n=2$, subject to the relations given below. Then $G$ is isomorphic to $N$.

(i) Defining relations for $S_{n}$ on $\{U, V\}$, omitting $V$ if $n=2$. 
(ii) $\Gamma^{q^{2}-1}=t^{2}=1, \Gamma^{t}=\Gamma^{-q}$.

(iii) If $n>2$ then $\left[\Gamma, U^{V}\right]=\left[t, U^{V}\right]=1$, and if $n>3$ then $[\Gamma, V U]=[t, V U]=1$.

(iv) $\left[\Gamma, \Gamma^{U}\right]=\left[t, t^{U}\right]=\left[\Gamma, t^{U}\right]=1$.

Proof. This is a standard wreath product presentation.

Theorem 8.2. The centraliser $H$ of $\nu=\nu_{1}(1)$ in $N$ has index $2\left(q^{2}-1\right) n$ in $N$ and is generated by the following elements:

(i) $U^{V}$ if $n>2$;

(ii) $V U$ if $n>3$;

(iii) if $q$ is even, then $t^{U}$, else $t^{U} \Delta^{\left(q^{2}-1\right) / 2}$;

(iv) if $n=2$ and $q \equiv 1 \bmod 3$ then $\Gamma^{q-1} \Gamma^{3 U}\left[t, \Gamma^{-1}\right]^{U}$, else $\Gamma^{q-1} \Gamma^{3 U}$;

(v) $\Gamma^{U} \Gamma^{-V^{2}}$ if $n>2$.

Proof. Since the matrix $\operatorname{diag}\left(a_{1}, a_{2}, \ldots, a_{2 n+1}\right)$ centralises $\nu$ if and only if $a_{1}=a_{2}=a_{2 n+1}$, these elements centralise $\nu$.

The orbit of $\nu$ under conjugation by $N$ is $\left\{\nu_{ \pm i}(\alpha) \mid 1 \leq i \leq n, \alpha \in \mathrm{GF}\left(q^{2}\right)^{\times}\right\}$, and has size $2\left(q^{2}-1\right) n$.

Consider the case $n>2$. Item (v) is the matrix $g=\operatorname{diag}\left(1,1, \omega^{-1}, \omega^{q}, \omega, \omega^{-q}, 1, \ldots, 1\right)$; clearly the conjugates of $g$ under the copy of $S_{n-1}$ generated by items (i) and (ii) generate a group $A$ of order $\left(q^{2}-1\right)^{n-2}$, namely

$$
\left\{\operatorname{diag}\left(1,1, a_{2}, a_{2}^{-q}, a_{3}, a_{3}^{-q}, \ldots, a_{n}, a_{n}^{-q}, 1\right) \mid a_{i} \in \mathrm{GF}\left(q^{2}\right)^{\times}, a_{2} a_{3} \ldots a_{n}=1\right\} .
$$

If $h$ is the matrix given by item (iii) then $[g, h]=\operatorname{diag}\left(1,1, \omega_{0}, \omega_{0}^{-1}, 1, \ldots, 1\right)$; this commutator and $A$ generate a group $B$ of order $(q-1)\left(q^{2}-1\right)^{n-2}$, namely

$$
\left\{\operatorname{diag}\left(1,1, a_{2}, a_{2}^{-q}, a_{3}, a_{3}^{-q}, \ldots, a_{n}, a_{n}^{-q}, 1\right) \mid a_{i} \in \mathrm{GF}\left(q^{2}\right)^{\times}, a_{2} a_{3} \ldots a_{n} \in \mathrm{GF}(q)^{\times}\right\} .
$$

Also, $\Gamma^{q-1} \Gamma^{3 U}=\operatorname{diag}\left(\omega^{1-q}, \omega^{1-q}, \omega^{-3}, \omega^{3 q}, 1, \ldots, 1, \omega^{1-q}\right)$; it and $B$ generate a group of order $\left(q^{2}-1\right)^{n-1}$.

Thus $H$ has order $\left(q^{2}-1\right)^{n-1} 2^{n-1}(n-1)$ !, the factor $2^{n-1}$ coming from conjugates of $h$, and the factor of $(n-1)$ ! from items (i) and (ii). So $H$ has index $2\left(q^{2}-1\right) n$ in $N$, as required.

The case $n=2$ is obvious.

Theorem 8.3. The centraliser of $\sigma=\sigma_{12}(1)$ in $N$ has index $2\left(q^{2}-1\right) n(n-1)$ in $N$ and is generated by the following elements:

(i) $U^{V^{2}}$ if $n>3$, and $V U U^{V}$ if $n>4$;

(ii) both $\Gamma^{V^{2}}$ and $t^{V^{2}}$ if $n>2$;

(iii) if $q$ is even, then $U^{t}$, else $U^{t} Z^{2}$;

(iv) $\Gamma \Gamma^{U}$.

Proof. The elements $\left\{\sigma_{ \pm i \pm j}(\alpha) \mid i \leq i \neq j \leq n, \alpha \in \mathrm{GF}\left(q^{2}\right)^{\times}\right\}$of $\mathrm{SU}(2 n+1, q)$, each of which may be written in exactly two ways in this form, are permuted transitively by $N$, forming an orbit of size $2\left(q^{2}-1\right) n(n-1)$.

Clearly the itemised elements centralise $\sigma$. The first two sets generate a copy of $\left(C_{q^{2}-1}\right.$ : $C_{2}$ ) ₹ $S_{n-2}$, and the last two a copy of $C_{q^{2}-1}: C_{2}$. Their direct product has the claimed index in $N$. 
Theorem 8.4. Let $q=p^{e}$ for a prime $p$ and let $n \geq 2$. Let $G$ be the group generated by $\{\nu, \sigma, \tau, \Gamma, t, U, V\}$, omitting $V$ if $n=2$, subject to the relations given below. Then $G$ is isomorphic to $\mathrm{SU}(2 n+1, q)$.

(i) Defining relations for $N=\langle\Gamma, t, U, V\rangle$ as in Theorem 8.1, but omitting relations (ii) of that theorem.

(ii) Relations that state that the elements listed in Theorem 8.2 centralise $\nu$.

(iii) Relations that state that the elements listed in Theorem 8.3 centralise $\sigma$, but omitting items (i) and (ii) of that theorem.

(iv) Defining relations for $\mathrm{SU}(3, q)$ on $\left\{\nu, \tau^{-1}, \Gamma^{-1}, t\right\}$ if $q>2$, and on $\left\{\nu, \nu^{\Gamma^{U}}, \Gamma^{-1}, t\right\}$ as in Section 4.3 if $q=2$.

(v) Defining relations for $\mathrm{SL}\left(2, q^{2}\right)$ on $\{\sigma, \Delta, W\}$, where $\Delta=\Gamma \Gamma^{-U}$, and $W=U$ if $q$ is even, else $W=U Z^{2}$.

(vi) The following instances of Steinberg relations:

(a) $\left[\nu, \nu^{U}\right]=\sigma^{-t^{U}} ; \quad\left(\left[\nu_{i}(\alpha), \nu_{j}(\beta)\right]=\sigma_{i,-j}\left(\psi^{-1} \alpha \beta^{q}\right)\right)$;

(b) if $q=4$ then $\left[\nu, \nu^{\Gamma U}\right]=\sigma^{\Gamma^{7} t^{U}} ; \quad\left(\left[\nu_{i}(\alpha), \nu_{j}\left(\omega^{2-q} \beta\right)\right]=\sigma_{i,-j}\left(\omega^{7} \alpha \beta^{q}\right)\right)$;

(c) $[\nu, \sigma]=1 ; \quad\left(\left[\nu_{i}(\alpha), \sigma_{i j}(\beta)\right]=1\right)$;

(d) if $q=4$ then $\left[\nu^{\Gamma}, \sigma\right]=1 ; \quad\left(\left[\nu_{i}\left(\omega^{2-q} \alpha\right), \sigma_{i j}(\beta)\right]=1\right)$;

(e) if $q$ is even, then $\left[\nu, \sigma^{U}\right]=\sigma^{\Gamma^{r} Z^{U}} \nu^{U}$, where $\omega^{r}=\omega^{q} /\left(\omega+\omega^{q}\right)$;

if $q$ is odd then $\left[\nu, \sigma^{U}\right]=\sigma^{\Gamma^{r} Z^{U}} \nu^{-U}$, where $\omega^{r}=\frac{1}{2} \omega^{-(q+1) / 2}$; (see (5) below);

(f) if $q=2$ then $\left[\nu, \sigma^{\Gamma U}\right]=\sigma^{\Gamma Z^{U}} \nu^{U^{\Gamma^{-1}}}$; (see (5) below with $\beta$ replaced by $\left.\omega \beta\right)$;

(g) if $n>2$ then $\left[\nu, \sigma^{V}\right]=1 ; \quad\left(\left[\nu_{i}(\alpha), \sigma_{j k}(\beta)\right]=1\right)$;

(h) $\left[\nu, \tau^{U}\right]=1 ; \quad($ see $(2)$ below);

(j) the Steinberg relations (v) of Theorem 7.7 .

The given relations are instances of the more general relations in parentheses, with $s=\alpha=\beta=1$ and $(i, j, k, l)=(1,2,3,4)$.

Proof. The relations omitted from (i) are supplied by (iv), so we may define $N$ as a subgroup of $G$. The relations omitted from (iii) may be retrieved using (i), (ii), and (a).

The Steinberg relations (j) involve $\Delta=\Gamma \Gamma^{-U}$ and $Z$ where $Z=t$ if $q$ is even, else $Z=t \Gamma^{(q+1) / 2}$.

In $\mathrm{SU}(2 n+1, q), N$ acts transitively by conjugation on $\left\{\nu_{ \pm i}(\alpha) \mid 1 \leq i \leq n, \alpha \in\right.$ $\left.\operatorname{GF}\left(q^{2}\right)^{\times}\right\}$, and on $\left\{\sigma_{ \pm i \pm j}(\alpha) \mid 1 \leq i \neq j \leq n, \alpha \in \mathrm{GF}\left(q^{2}\right)^{\times}\right\}$, so these elements may be defined in $G$, by (i), (ii), and (iii), and the omitted relations.

In $\mathrm{SU}(2 n+1, q)$ the centraliser of $\tau$ in $N$ is the direct product of the cyclic group of order $q+1$ generated by $\Gamma^{q-1}$ with the subgroup of $N$ that fixes $e_{1}$ and $f_{1}$, and it has order $2^{n-1}(q+1)\left(q^{2}-1\right)^{n-1}(n-1)$ !. This direct product has index $2(q-1) n$ in $N$, which corresponds to the fact that $N$ acts transitively by conjugation on $\left\{\tau_{ \pm i}(s)|1 \leq| i \mid \leq\right.$ $\left.n, s \in \mathrm{GF}(q)^{\times}\right\}$. It follows from (i), (ii), (iv) and (j) that this group centralises $\tau$ in $G$, so $\left\{\tau_{ \pm i}(s)|1 \leq| i \mid \leq n, s \in \mathrm{GF}(q)^{\times}\right\}$may be be defined in $G$, together with the action of $N$ on these elements.

The normaliser $N$ of our chosen torus in $\mathrm{SU}(2 n+1, q)$ contains the normaliser of our chosen torus in $\mathrm{SU}(2 n, q)$ as a subgroup of index $q+1$; but the centraliser of $\sigma$ in $N$ is correspondingly larger, so the definitions of the Steinberg generators $\sigma_{i j}(\alpha)$ are essentially the same in both cases. Thus $G$ contains a copy of $\mathrm{SU}(2 n, q)$, and our first objective is 
to prove that the Steinberg relations involving the generators $\nu_{i}(\alpha)$, and not subsumed in (iv), are satisfied. These relations are as follows, where $|i|,|j|$, and $|k|$ are distinct:

(1) $\left[\nu_{i}(\alpha), \sigma_{i j}(\beta)\right]=1$

(2) $\left[\nu_{i}(\alpha), \tau_{j}(s)\right]=1$

(3) $\left[\nu_{i}(\alpha), \nu_{j}(\beta)\right]=\sigma_{i,-j}\left(\psi^{-1} \alpha \beta^{q}\right)$;

(4) $\left[\nu_{i}(\alpha), \sigma_{j k}(\beta)\right]=1$ if $n>2$;

(5) $\left[\nu_{i}(\alpha), \sigma_{j i}(\beta)\right]=\sigma_{i,-j}\left(\psi^{-1}(\phi+1) \alpha^{q+1} \beta^{q}\right) \nu_{j}(-\alpha \beta)$.

We have omitted Steinberg relations whose LHS involves the same suffix twice, but with opposite signs, since $\sigma_{-i, j}(\alpha)=\sigma_{-j, i}\left(\alpha^{q}\right)$.

We may always assume that $(i, j, k)=(1,2,3)$ since conjugating a Steinberg generator by $t_{i}$ changes the sign of suffices of the form $\pm i$ and keeps all other suffices fixed.

We now prove relation (1). Let $P$ be the set of pairs $(\alpha, \beta)$ for which (1) holds in $G$ with $(i, j)=(1,2)$. By relation $(\mathrm{c}),(1,1) \in P$. Clearly if $\left(\alpha, \beta_{1}\right)$ and $\left(\alpha, \beta_{2}\right)$ lie in $P$ then so does $\left(\alpha, \beta_{1}+\beta_{2}\right)$. If $\left(\alpha_{1}, \beta\right)$ and $\left(\alpha_{2}, \beta\right)$ lie in $P$ then so does $\left(\alpha_{1}+\alpha_{2}, \beta\right)$ : for $\nu_{1}\left(\alpha_{1}+\alpha_{2}, \beta\right) \equiv \nu_{1}\left(\alpha_{1}, \beta\right) \nu_{1}\left(\alpha_{2}, \beta\right) \bmod \left\langle\left\{\tau_{1}(s) \mid s \in \mathrm{GF}(q)\right\}\right\rangle$, and $\tau_{1}(s)$ commutes with $\sigma_{12}(\beta)$ by Theorem $7.7(\mathrm{v})(\mathrm{a})$. Conjugating by $\Gamma_{1}$ and by $\Gamma_{2}$ shows that if $(\alpha, \beta) \in P$ then $\left(\omega^{-q+2} \alpha, \omega \beta\right) \in P$, and $\left(\omega^{-q+1}, \omega^{-1} \beta\right) \in P$, so $\left(\omega^{-2 q+3} \alpha, \beta\right) \in P$. But $\operatorname{gcd}(-2 q+3, q-1)=$ 1 and $\operatorname{gcd}(-2 q+3, q+1)=\operatorname{gcd}(5, q+1)$, so $\omega^{-2 q+3}$ generates $\operatorname{GF}\left(q^{2}\right)$ as a field if $q \neq 4$. So if $q \neq 4$ and $(\alpha, \beta) \in P$ for some $(\alpha, \beta)$, then $(\alpha, \beta) \in P$ for that value of $\beta$ and all $\alpha$, and hence for all $(\alpha, \beta)$. Now let $q=4$. By the action of $\Gamma_{j}^{-q-1}$ we see that if $(\alpha, \beta) \in P$ then $\left(\alpha, \omega_{0} \beta\right) \in P$; and since $\omega^{2 q-3}=\omega^{5}=\omega_{0}$, it follows that $\left(\omega_{0} \alpha, \beta\right) \in P$. Now by relation $(\mathrm{d})$ we see that, in addition to $(1,1)$, also $\left(\omega^{-2}, 1\right) \in P$, and hence $(\alpha, 1) \in P$ for all $\alpha \in \operatorname{GF}\left(q^{2}\right)$. It follows that $(\alpha, \beta) \in P$ for all $(\alpha, \beta)$, and (1) is proved.

We now prove relation (2). Let $P$ be the set of pairs $(\alpha, s)$ for which $(2)$ holds in $G$ with $(i, j)=(1,2)$. Clearly if $\left(\alpha_{1}, s\right) \in P$ and $\left(\alpha_{2}, s\right) \in P$ then $\left(\alpha_{1}+\alpha_{2}, s\right) \in P$, as in the proof of $(1)$, and if $\left(\alpha, s_{1}\right) \in P$ and $\left(\alpha, s_{2}\right) \in P$, then $\left(\alpha, s_{1}+s_{2}\right) \in P$. Conjugating by $\Gamma_{1}$ and by $\Gamma_{2}$ shows that if $(\alpha, s) \in P$ then $\left(\omega^{-q+2} \alpha, s\right) \in P$, and $\left(\omega^{-q+1} \alpha, \omega_{0} s\right) \in P$. If $q \neq 2$ then $\omega^{-q+2}$ generates $\operatorname{GF}\left(q^{2}\right)$ as a field; if $q=2$ then $\omega_{0}=1$, and $\omega^{-q+1}$ generates $\operatorname{GF}\left(q^{2}\right)$ as a field. So in either case if $(\alpha, s) \in P$ for one value of $(\alpha, s)$ then $(\alpha, s) \in P$ for that value of $s$ and all values of $\alpha$, and hence (if $s \neq 0$ ) for all values of $s$ and all values of $\alpha$. But $(1,1) \in P$, by relation (h), so (2) is proved.

We now prove relation (3). Let $P$ be the set of pairs $(\alpha, \beta)$ for which (3) holds in $G$ with $(i, j)=(1,2)$. Relation (a) implies that $(1,1) \in P$. As in the proof of $(1)$, it follows from (2) that if $\left(\alpha_{1}, \beta\right) \in P$ and $\left(\alpha_{2}, \beta\right) \in P$ then $\left(\alpha_{1}+\alpha_{2}, \beta\right) \in P$, and similarly with the roles of $\alpha$ and $\beta$ reversed. Conjugating by $\Gamma_{1}^{q+1}$, one sees that if $(\alpha, \beta) \in P$ then $\left(\omega_{0} \alpha, \beta\right) \in P$ and similarly $\left(\alpha, \omega_{0} \beta\right) \in P$. Now let $T$ be the set of $\alpha \in \operatorname{GF}\left(q^{2}\right)$ such that $(\alpha, \beta) \in P$ for all $\beta \in \mathrm{GF}\left(q^{2}\right)$. Since $T$ is closed under addition and (using conjugation by $\left.\Gamma_{1}\right)$ under multiplication by $\omega^{q-1}$, and since $\omega^{q-1}$ generates $\operatorname{GF}\left(q^{2}\right)$ as a field, $(3)$ holds if $T \neq\{0\}$. So we are reduced to the case in which, for every $\alpha \in \operatorname{GF}\left(q^{2}\right)^{\times}$, the pair $(\alpha, \beta)$ lies in $P$ for exactly $q-1$ elements $\beta \in \operatorname{GF}\left(q^{2}\right)^{\times}$, making $\left(q^{2}-1\right)(q-1)$ such pairs in all. Now conjugating by $\Gamma_{1}$ and $\Gamma_{2}$ shows that if $(\alpha, \beta) \in P$ then $\left(\omega^{-q+2} \alpha, \omega^{-q+1} \beta\right) \in P$ and $\left(\omega^{-q+1} \alpha, \omega^{-q+2} \beta\right) \in P$. Since $\omega^{q+1}=\omega_{0}$, it follows that $\left(\omega^{2} \alpha, \omega^{3} \beta\right) \in P$, and $\left(\omega^{3} \alpha, \omega^{2} \beta\right) \in$ $P$. If $q^{2}-1$ is not a multiple of 5 then the pairs $(2,3)$ and $(3,2)$ generate $C_{q^{2}-1} \times C_{q^{2}-1}$, so we may now assume that $q^{2}-1$ is a multiple of 5 . Now $P$ contains at least $\left(q^{2}-1\right)^{2} / 5$ 
pairs $(\alpha, \beta)$ with $\alpha \beta \neq 0$. But if (3) does not hold then $P$ contains exactly $\left(q^{2}-1\right)(q-1)$ such pairs. Thus (3) follows from one instance, unless $q=4$, when a second instance is required, as in relation $(\mathrm{b})$, where $(\alpha, \beta)=\left(1, \omega^{2}\right)$.

We now prove relation (4). We may assume that $(i, j, k)=(1,2,3)$ : the result follows trivially from the case $\alpha=\beta=1$, as in relation (g), by conjugating with $\Gamma_{1}$ and $\Gamma_{2}$.

We now prove relation (5). Note that $\sigma_{i,-j}\left(\psi^{-1}(\phi+1) \alpha^{q+1} \beta^{q}\right)$ and $\nu_{j}(-\alpha \beta)$ commute by (4). As usual, we restrict to the case $(i, j)=(1,2)$. Let $P$ be the set of pairs $(\alpha, \beta)$ for which (5) holds in $G$ with $(i, j)=(1,2)$. Conjugating by $\Gamma_{1}$ or $\Gamma_{2}$ shows that if $(\alpha, \beta) \in P$ then $\left(\omega^{2-q} \alpha, \omega \beta\right) \in P$, and $\left(\omega^{1-q} \alpha, \omega^{-1} \beta\right) \in P$. Thus if $(\alpha, \beta) \in P$ then $\left(\omega^{3-2 q} \alpha, \beta\right) \in P$. It is also clear, from the above information, that if $\left(\alpha_{1}, \beta\right) \in P$ and $\left(\alpha_{2}, \beta\right) \in P$ then $\left(\alpha_{1}+\alpha_{2}, \beta\right) \in P$. If $q \neq 2$ then $\omega^{2-q}$ generates $\operatorname{GF}\left(q^{2}\right)$ as a field, and hence (5) follows from any one instance (with $\alpha \beta \neq 0$ ), such as relation (e), which shows that $(1,1) \in P$. If $q=2$ then relation (f), which states that $(1, \omega) \in P$, completes the proof in this case.

Thus the subgroup $H$ of $G$ generated by the Steinberg generators is isomorphic to $\mathrm{SU}(2 n+1, q)$, and it remains to prove that this subgroup equals $G$. By (iii), $\Gamma$ and $t$ lie in $H$. Also, $U$ and $V$ lie in $H$, as in the case of $\mathrm{SU}(2 n, q)$. Thus $G=H$, as required.

8.3. A presentation for $\operatorname{PSU}(2 n+1, q)$. The centre of $\mathrm{SU}(2 n+1, q)$ has order $\operatorname{gcd}(q+$ $1,2 n+1)$; if it is non-trivial, then it is generated by $(\Gamma Z V)^{2 n(q+1) / \operatorname{gcd}(q+1,2 n+1)}$.

8.4. Standard generators for $\mathrm{SU}(2 n+1, q)$. In Table 1 of [10, 21] the standard generators for $\mathrm{SU}(2 n+1, q)$ are labelled $s, t, \delta, u, v, x, y$. Observe that $s=Z^{-1} ; t=\tau^{-1} ; \delta=$ $\Gamma^{-(q+1)} ; u=U ; v=V ; x=\nu^{\left(V^{-1}\right)} ;$ and $y=\left(\Gamma^{-1}\right)^{\left(V^{-1}\right)}$. If $q$ is odd then the presentation generator $t=\Gamma^{\left(\left(q^{2}+q\right) / 2\right)} s^{-1}$, else $t=s$. Also $\sigma=\left[\nu^{t U}, \nu^{t}\right]^{t}$, as a word in the presentation generators $\nu, t$, and $U$, so it can be written as an explicit word in the standard generators.

\section{A PRESENTATION FOR $\Omega^{+}(2 n, q)$ FOR $n>1$}

\subsection{Odd characteristic.}

9.1.1. Generators and notation. Let $q=p^{e}$ for an odd prime $p$. Let $\omega$ be a primitive element of $\operatorname{GF}(q)$.

Let $n>1$. We take a hyperbolic basis $\left(e_{1}, f_{1}, \ldots, e_{n}, f_{n}\right)$ of the natural module. We define the following elements of $\mathrm{SO}^{+}(2 n, q)$, where $1 \leq i, j \leq n$ and $i \neq j$, and $s \in \operatorname{GF}(q)$.

$$
\begin{aligned}
& \delta_{i}=\left(e_{i} \mapsto \omega^{-1} e_{i}, f_{i} \mapsto \omega f_{i}\right) ; \\
& \Delta_{i j}=\delta_{i} \delta_{j}^{-1} ; \\
& \Delta_{-i, j}=\delta_{i}^{-1} \delta_{j}^{-1} ; \\
& \Delta_{i,-j}=\delta_{i} \delta_{j} ; \\
& \Delta_{-i,-j}=\delta_{i}^{-1} \delta_{j} ; \\
& \sigma_{i j}(s)=\left(e_{i} \mapsto e_{i}+s e_{j}, f_{j} \mapsto f_{j}-s f_{i}\right) ; \\
& \sigma_{i,-j}(s)=\left(e_{i} \mapsto e_{i}+s f_{j}, e_{j} \mapsto e_{j}-s f_{i}\right) ; \\
& \sigma_{-i, j}(s)=\left(f_{i} \mapsto f_{i}+s e_{j}, f_{j} \mapsto f_{j}-s e_{i}\right) ; \\
& \sigma_{-i,-j}(s)=\sigma_{j i}(-s) ; \\
& Z_{i j}=\left(e_{i}, f_{i}\right)\left(e_{j}, f_{j}\right) ; \\
& U=\left(e_{1}, e_{2}\right)\left(f_{1}, f_{2}\right) ; \\
& U^{\prime}=\left(e_{1}, e_{2}\right)^{-}\left(f_{1}, f_{2}\right)^{-} ;
\end{aligned}
$$




$$
\begin{aligned}
& V=\left(e_{1}, e_{2}, \ldots, e_{n}\right)\left(f_{1}, f_{2}, \ldots, f_{n}\right) \\
& V^{\prime}=\left(e_{1}, e_{2}, \ldots, e_{n}\right)^{\epsilon_{n}}\left(f_{1}, f_{2}, \ldots, f_{n}\right)^{\epsilon_{n}} .
\end{aligned}
$$

All these elements of $\mathrm{SO}^{+}(2 n, q)$ centralise those basis elements that they are not stated to move. All lie in $\Omega^{+}(2 n, q)$ except for $\delta_{i}$, and $U$ if $q \equiv 3 \bmod 4$, and $V$ if $n$ is even and $q \equiv 3 \bmod 4$. These statements, except for those involving $V$ and $V^{\prime}$, follow from the analysis of $\Omega^{+}(4, q)$ below. Since $V^{\prime}$ is a product of conjugates of $U^{\prime}$, it lies in $\Omega^{+}(2 n, q)$. Also $U=U^{\prime} \delta_{1}^{(q-1) / 2}$ and $V=V^{\prime} \delta_{1}^{(q-1) / 2}$ if $n$ is even, so the statements concerning $V$ follow.

9.1.2. A presentation for $\Omega^{+}(4, q)$. Since $\Omega^{+}(4, q)$ is the central product of two copies of $\mathrm{SL}(2, q)$, there is no difficulty in writing down the required presentation, but we look more closely at this case as a preparation for the general case.

Consider the spaces $\left\langle e_{1}, e_{2}\right\rangle$ and $\left\langle f_{1}, f_{2}\right\rangle$. They are fixed by $\left\{\sigma_{12}(1), \Delta_{12}, U^{\prime}\right\}$. These elements act on $\left\langle e_{1}, e_{2}\right\rangle$ by the matrices $\left(\begin{array}{ll}1 & 1 \\ 0 & 1\end{array}\right),\left(\begin{array}{cc}\omega_{0}^{-1} & 0 \\ 0 & \omega\end{array}\right)$, and $\left(\begin{array}{cc}0 & 1 \\ -1 & 0\end{array}\right)$ respectively; they act on $\left\langle f_{1}, f_{2}\right\rangle$ by the inverse transpose of these matrices. So these elements generate a copy of $\operatorname{SL}(2, q)$. Now consider the spaces $\left\langle e_{1}, f_{2}\right\rangle$ and $\left\langle e_{2}, f_{1}\right\rangle$. These are fixed by $\left\{\sigma_{1,-2}(1), \Delta_{1,-2}, Z_{12} U^{\prime}\right\}$. These elements act on $\left\langle e_{1}, f_{2}\right\rangle$ as the above three matrices, and as the inverse transpose on $\left\langle e_{2}, f_{1}\right\rangle$, thus generating a second copy of $\mathrm{SL}(2, q)$. The two copies of $\operatorname{SL}(2, q)$ commute with each other, and $\Delta_{12}^{(q-1) / 2}=\Delta_{1,-2}^{(q-1) / 2}=-I_{4}$. This gives the required presentation on $\left\{\sigma_{12}(1), \Delta_{12}, U^{\prime}, \sigma_{1,-2}(1), \Delta_{1,-2}, Z_{12} U^{\prime}\right\}$, omitting $\Delta_{12}$ and $\Delta_{1,-2}$ if $e=1$.

9.1.3. A presentation for $\Omega^{+}(2 n, q)$ for $n \geq 3$ and $e>1$. We give a presentation for $\Omega^{+}(2 n, q)$ on the generating set $\left\{\sigma=\sigma_{12}(1), \Delta=\Delta_{12}, Z=Z_{12}, U^{\prime}, V^{\prime}\right\}$.

Let $N_{1}$ be the subgroup of $\Omega^{+}(2 n, q)$ generated by $\left\{Z, U^{\prime}, V^{\prime}\right\}$. This is an extension of an elementary abelian 2-group $A$ of rank $2(n-1)$ by a copy of $S_{n}$. Here $A$ is the direct product of a subgroup of rank $n-1$, generated by $U^{\prime 2}$ and its conjugates, with a second subgroup of the same rank generated by $Z$ and its conjugates. So if $B$ is the copy of the four-group generated by the transformations $\left(e_{1} \mapsto-e_{1}, f_{1} \mapsto-f_{1}\right)$ and $\left(e_{1} \mapsto f_{1}, f_{1} \mapsto e_{1}\right)$, the other basis vectors being fixed, then $N_{1}$ is the subgroup of $B$ ? $S_{n}$ of index 4 consisting of the elements of determinant 1 and spinor norm 1.

Theorem 9.1. Let $n \geq 3$. Let $G$ be the group generated by $\left\{Z, U^{\prime}, V^{\prime}\right\}$ subject to the relations given below. Then $G$ is isomorphic to $N_{1}$.

(i) Defining relations for $\left\langle U^{\prime}, V^{\prime}\right\rangle$ as in Theorems 2.2 and 2.3 .

(ii) If $n>3$ then $\left[Z, U^{\prime V^{\prime 2}}\right]=1$.

(iii) If $n>4$ then $\left[Z, V^{\prime} U^{\prime} U^{\prime V^{\prime}}\right]=1$.

(iv) $Z^{2}=1$

(v) $\left[Z, U^{\prime 2 V^{\prime}}\right]=1$.

(vi) $Z Z^{V^{\prime}}=Z^{V^{\prime} U^{\prime}}$.

(vii) $\left[Z, U^{\prime}\right]=1$.

(viii) $\left[Z, Z^{V^{\prime}}\right]=1$.

(ix) If $n>3$ then $\left[Z, Z^{V^{\prime 2}}\right]=1$.

Proof. Relations (ii) and (iii) state that $Z$ is centralised by the subgroup of $\left\langle U^{\prime}, V^{\prime}\right\rangle$ that centralises 1 and 2 as signed permutations, and also by $U^{\prime 2}$. These relations, together 
with (v), also imply that $Z$ is centralised by all $\left\langle U^{\prime}, V^{\prime}\right\rangle$-conjugates of $U^{\prime 2}$, so we may label these conjugates as $Z_{i j}$, where $1 \leq i \neq j \leq n$. Now (vii) implies that $Z_{12}=Z_{21}$, and hence, by conjugation, that $Z_{i j}=Z_{j i}$ for all $i \neq j$.

It follows similarly from (viii) and (ix) that all $Z_{i j}$ commute, and hence, by (iv), generate an elementary abelian 2-group. Also, (vi) states that $Z_{12} Z_{23}=Z_{13}$, so $Z_{i j} Z_{j k}=Z_{i k}$ for any three distinct values of $i, j, k$, and so the subgroup of $G$ generated by the $Z_{i j}$ is generated by $\left\{Z_{12}, Z_{23}, \ldots, Z_{n-1, n}\right\}$, and hence has order at most $2^{n-1}$. Thus $G$ is a split extension of a normal subgroup of order at most $2^{n-1}$ by the group $\left\langle U^{\prime}, V^{\prime}\right\rangle$ of order $2^{n-1} n$ !, by (i). But $N_{1}$, as a subgroup of $\Omega^{+}(2 n, q)$, has order $2^{2 n-2} n$ !, and the result follows.

Let $N=\left\langle\Delta, Z, U^{\prime}, V^{\prime}\right\rangle \leq \Omega^{+}(2 n, q)$. It is easy to see that the normal subgroup of $N$ generated as normal subgroup by $\Delta$, and as subgroup by $\left\{\Delta_{ \pm i, \pm j}|| i|\neq| j \mid\right\}$, is generated by $\left\{\Delta_{1,-2}, \Delta_{12}, \Delta_{23}, \ldots, \Delta_{n-1, n}\right\}$, and has order $(q-1)^{n} / 2$, the factor of $1 / 2$ coming from the relation $\Delta_{12}^{(q-1) / 2}=\Delta_{1,-2}^{(q-1) / 2}$. Since this normal subgroup contains $U^{\prime 2}$, it follows that $N$ has order $(q-1)^{n} 2^{n-2} n$ !.

Theorem 9.2. Let $q=p^{e}$ for an odd prime $p$ and $e>1$, and let $n \geq 3$. Let $G$ be the group generated by $\left\{\Delta, Z, U^{\prime}, V^{\prime}\right\}$ subject to the relations given below. Then $G$ is isomorphic to $N$.

(i) Defining relations for $N_{1}=\left\langle Z, U^{\prime}, V^{\prime}\right\rangle$ as in Theorem 9.1,

(ii) If $n>3$ then $\left[\Delta, U^{\prime V^{\prime 2}}\right]=1$.

(iii) If $n>4$ then $\left[\Delta, V^{\prime} U^{\prime} U^{\prime V^{\prime}}\right]=1$.

(iv) If $n>3$ then $\left[\Delta, Z^{V^{\prime 2}}\right]=1$.

(v) $\Delta^{U^{\prime}}=\Delta^{-1}$, and $\left[\Delta, U^{\prime 2 V}\right]=1$.

(vi) $\Delta^{(q-1) / 2}=U^{\prime 2}$.

(vii) $\Delta \Delta^{V^{\prime}}=\Delta^{V^{\prime} U^{\prime}}$.

(viii) $\left[\Delta, \Delta^{V^{\prime}}\right]=1$.

(ix) If $n>3$ then $\left[\Delta, \Delta^{V^{\prime 2}}\right]=1$.

(x) $\Delta^{Z}=\Delta^{-1}$.

Proof. We first work in $\Omega^{+}(2 n, q)$. Since $n>2, N_{1}$ permutes transitively the elements $\Delta_{ \pm i, \pm j}$. Here $\left\langle U^{\prime}, V^{\prime}\right\rangle$ permutes the suffices as unsigned permutations, so $U^{\prime 2}$ centralises, and $Z_{i j}$ sends $i$ to $-i$, and $j$ to $-j$. Since $\Delta_{j i}=\Delta_{-i,-j}$ these $4 n(n-1)$ symbols define $2 n(n-1)$ distinct matrices. Now $\Delta=\Delta_{12}$ is centralised by the subgroup of $N_{1}$ that centralises $\left\langle e_{1}, f_{1}, e_{2}, f_{2}\right\rangle$ and has order $2^{2(n-3)}(n-2)$ !, and also by $Z U^{\prime}$ and $U^{\prime 2 V^{\prime}}$, which contribute an additional factor of 8 to the order of the centraliser. Thus the centraliser of $\Delta$ in $N_{1}$ has order $2^{2 n-3}(n-2)$ ! and index $2 n(n-1)$ as required.

We now work in the presented group $G$. By the above remarks, and using relations (i) to (v) and (x), we may define the elements $\Delta_{ \pm i, \pm j}$ in $G$ as the $N_{1}$-conjugates of $\Delta$. Relation (v) states that $\Delta_{21}=\Delta_{12}^{-1}$, and hence $\Delta_{i j}=\Delta_{j i}^{-1}$ whenever $|i| \neq|j|$. Relations (viii) and (ix) state that $\Delta_{12}$ commutes with $\Delta_{23}$, and with $\Delta_{34}$ if $n>3$, so the group $K$ generated by $\left\{\Delta_{i j} \mid i, j>0, i \neq j\right\}$ is abelian. Since $\Delta_{-1,-2}=\Delta_{12}^{-1}$ by (x), it follows that $\Delta_{-i,-j}=\Delta_{i j}^{-1}$ if $|i| \neq|j|$. Relation (vii) states that $\Delta_{12} \Delta_{23}=\Delta_{13}$, and hence $\Delta_{i j} \Delta_{j k}=\Delta_{i k}$ whenever $|i|,|j|$, and $|k|$ are distinct. It is now easy to prove, since $n \geq 3$, that the group $H$ generated by $\left\{\Delta_{ \pm i \pm j}|| i|\neq| j \mid\right\}$ is abelian, and that $K$ is generated by 
$\left\{\Delta_{i, i+1} \mid 1 \leq i<n\right\}$. Since $\Delta_{i,-j}=\Delta_{i 1} \Delta_{1,-2} \Delta_{-2,-j}=\Delta_{i 1} \Delta_{1,-2} \Delta_{2, j}^{-1}$ if $|i| \neq|j|$, it follows that $H$ is generated by $\left\{\Delta_{1,-2}, \Delta_{12}, \Delta_{23}, \ldots, \Delta_{n-1, n}\right\}$. Now $H$ has exponent dividing $q-1$ by (vi), and order dividing $(q-1)^{n} / 2$, the factor of $1 / 2$ coming from the fact that $\Delta_{12}^{(q-1) / 2}=U^{\prime 2}=U^{\prime 2 Z}=\Delta_{1,-2}^{(q-1) / 2}$. Since the corresponding subgroup of $\Omega^{+}(2 n, q)$ has this order, so does $H$. The result follows.

Theorem 9.3. Let $q=p^{e}$ for an odd prime $p$ and $e>1$, and let $n \geq 3$. The centraliser of $\sigma=\sigma_{12}(1)$ in $N$ has order $(q-1)^{n-1} 2^{n-3}(n-2)$ ! and is generated by the following elements:

(i) $U^{\prime V^{\prime 2}}$ if $n>3$;

(ii) $V^{\prime} U^{\prime-1} U^{\prime-V^{\prime}}$ if $n>4$;

(iii) $Z^{V^{\prime 2}}$ if $n>3$;

(iv) $\Delta^{V^{\prime 2}}$ if $n>3$

(v) $\Delta^{Z^{V^{\prime}}}$;

(vi) $Z U^{\prime}$;

(vii) $\Delta^{U^{\prime} V^{\prime}} \Delta^{V^{\prime}}$ if $n<5$.

Proof. Observe that $N$ acts transitively by conjugation on $\left\{\sigma_{i j}(s)|| i|\neq| j \mid, s \in \mathrm{GF}(q)^{\times}\right\}$, a set of size $2 n(n-1)(q-1)$, as follows. If $g \in\left\langle U^{\prime}, V^{\prime}\right\rangle$ sends $i$ to $\epsilon_{1} k$, and sends $j$ to $\epsilon_{2} l$, where $\epsilon_{1}, \epsilon_{2}= \pm 1$, then $\sigma_{i j}(s)^{g}=\sigma_{k l}\left(\epsilon_{1} \epsilon_{2} s\right)$; and $Z_{i j}$ acts on the suffices, interchanging $i$ with $-i$, and $j$ with $-j$, fixing all other suffices and $s$. The action of $\Delta_{i j}$ follows from the formulae $\sigma_{i j}(s)^{\delta_{i}}=\sigma_{i j}(s \omega)$ and $\sigma_{i j}(s)^{\delta_{j}}=\sigma_{i j}\left(s \omega^{-1}\right)$.

Items (v) and (vii) equal $\delta_{1} \delta_{2}$ and $\delta_{1} \delta_{2} \delta_{3}^{-2}$ respectively, while item (vi) centralises $\sigma$ since $\sigma_{12}(s)=\sigma_{-2,-1}(-s)$. The other itemised elements clearly centralise $\sigma$. It remains to prove that the group generated by the itemised elements has the claimed order.

Suppose first that $n=3$. Clearly items (v) and (vii) generate a group of order $(q-1)^{2} / 2$, and with item (vi) generate a group of order $(q-1)^{2}$, as required.

Now let $n>3$. The centraliser of $\sigma$ in $N$ is the direct product of the centraliser $C$ in $N$ of $\left\langle e_{1}, f_{1}, e_{2}, f_{2}\right\rangle$, which has order $(q-1)^{n-2} 2^{n-4}(n-2)$ !, with a copy $Q$ of $Q_{2(q-1)}$, namely $\left\langle\Delta_{1,-2}, Z_{12} U^{\prime}\right\rangle$. For this subgroup centralises $\sigma$, and has order $(q-1)^{n-1} 2^{n-3}(n-2)$ !. These generators of $Q$ are supplied by items (v) and (vi). Observe that $C$ is generated by the union of the following sets: the elements $\Delta_{k, k+1}$ for $k>2$, supplied by item (iv) and its conjugates under items (i) and (ii), which generate a group of order $(q-1)^{n-3}$; the elements $Z_{k, k+1}$ for $k>2$, supplied by item (iii) and its conjugates under items (i) and (ii), which contribute a factor of $2^{n-3}$; the elements in items (i) and (ii), which contribute a factor of $(n-2)$ !; and $\delta_{3}^{2}$, which contributes the remaining factor of $(q-1) / 2$. If $n>4$ then $\delta_{3}^{2}=\Delta^{V^{\prime 2}} \Delta^{W}$, where $W=V^{\prime 2} Z^{V^{\prime 3}}$, so this generator is not required; if $n=4$ then this element is supplied by items (v) and (vii); that is, by $\delta_{1} \delta_{2}$ and $\delta_{1} \delta_{2} \delta_{3}^{-2}$.

Theorem 9.4. Let $q=p^{e}$ for an odd prime $p$ and $e>1$, and let $n \geq 3$. Let $G$ be the group generated by $\left\{\sigma, \Delta, Z, U^{\prime}, V^{\prime}\right\}$ subject to the relations given below. Then $G$ is isomorphic to $\Omega^{+}(2 n, q)$.

(i) Defining relations for $N=\left\langle\Delta, Z, U^{\prime}, V^{\prime}\right\rangle$ as in Theorem 9.2 , but omitting relation (vi) of that theorem. 
(ii) Relations that state that the elements listed in Theorem 9.3 centralise $\sigma$.

(iii) Relations that present $\mathrm{SL}(2, q)$ on $\left\{\sigma, \Delta, U^{\prime}\right\}$.

(iv) The following instances of Steinberg relations:

(a) $\left[\sigma, \sigma^{V^{\prime}}\right]=\sigma^{V^{\prime} U^{\prime-1}} ; \quad\left(\left[\sigma_{i j}(s), \sigma_{j k}(t)\right]=\sigma_{i k}(s t)\right)$;

(b) $\left[\sigma, \sigma^{V^{\prime} U^{\prime-1}}\right]=1 ; \quad\left(\left[\sigma_{i j}(s), \sigma_{i k}(t)\right]=1\right)$;

(c) $\left[\sigma, \sigma^{W}\right]=1$, where $W=U^{\prime V^{\prime} U^{\prime-1}} ; \quad\left(\left[\sigma_{i j}(s), \sigma_{k j}(t)\right]=1\right)$;

(d) if $n>3$ then $\left[\sigma, \sigma^{V^{\prime 2}}\right]=1 ; \quad\left(\left[\sigma_{i j}(s), \sigma_{k l}(t)\right]=1\right)$;

(e) $\left[\sigma, \sigma^{Z^{V}}\right]=1 ; \quad\left(\left[\sigma_{i j}(s), \sigma_{i,-j}(t)\right]=1\right)$;

(f) if $n=4$ then $\left[\sigma, \sigma^{Z^{V} V^{2}}\right]=1 ; \quad\left(\left[\sigma_{i j}(s), \sigma_{k,-l}(t)\right]=1\right)$.

The given relations are instances of the more general relations in parentheses, with $s=t=1$ and $(i, j, k, l)=(1,2,3,4)$.

Proof. The relation omitted from (i) is supplied by (iii).

By (i) we may define the elements $Z_{i j}$ and $\Delta_{i j}$ in $G$. Since $N$ acts transitively, in $\Omega^{+}(2 n, q)$, by conjugation on the set of all root elements $\sigma_{i j}(s)$, where $s \neq 0$ and $|i| \neq|j|$, we may define $\sigma_{i j}(s)$ in $G$ by the same conjugation, by (ii).

Recall that $\Omega^{+}(4, q)$ is isomorphic to the direct product of two copies of $\operatorname{SL}(2, q)$ with the centres amalgamated, the natural representation of $\Omega^{+}(4, q)$ being the tensor product of two copies of the natural representation of $\operatorname{SL}(2, q)$. Here $\left\langle\sigma_{12}, \Delta_{1,-2}, U^{\prime}\right\rangle$ is one copy of $\operatorname{SL}(2, q)$, and $\left\langle\sigma_{1,-2}, \Delta_{12}, Z_{12} U^{\prime}\right\rangle$ is the other. The first of these subgroups in $G$ is isomorphic to $\operatorname{SL}(2, q)$ by (iii), and, since $Z_{12} U^{\prime}=U^{\prime Z_{23}}$, so is the second, by conjugation with $Z_{23}$. By (e), (i) and (ii), these subgroups commute with each other. The amalgamation of the centres follows from the presentation for $N$. It follows that, in $G$, the subgroup $\left\langle\sigma_{ \pm 1, \pm 2}(1), \Delta_{12}, \Delta_{1,-2}, Z, U^{\prime}\right\rangle$ is isomorphic to $\Omega^{+}(4, q)$.

The parenthetical Steinberg relations under each of the headings (a) to (d) all follow from one example, by conjugation with $N$.

All remaining Steinberg relations may be deduced as follows. Those that only involve positive suffices follow easily, as in the case of $\operatorname{SL}(n, q)$. Indeed, $\left\langle\sigma, \Delta, U^{\prime}, V^{\prime}\right\rangle$ is isomorphic to $\operatorname{SL}(n, q)$. Since $\left\langle\sigma_{ \pm 1, \pm 2}(1), \Delta_{12}, \Delta_{1,-2}, Z, U^{\prime}\right\rangle$ is isomorphic, in $G$, to $\Omega^{+}(4, q)$, those Steinberg relations that only involve two suffices, with various signs, all hold in $G$. Relations that involve four different suffices, with various signs, follow from the one case (d) by conjugation, except in the case $n=4$, when the extra relation (f) is needed, as we can only change the signs of the suffices two at a time. It remains to consider the case when three suffices occur: namely, the LHS of the relation is of the form $\left[\sigma_{i j}(s), \sigma_{u v}(t)\right]$, where $\{a, b, c\}:=\{|i|,|j|,|u|,|v|\}$ forms a set of size 3 . Conjugating by $Z_{a b}$, or $Z_{b c}$, or $Z_{a c}$ changes the signs of any two of these suffices at all their appearances. Also the relation $\sigma_{i j}(s)=\sigma_{-j,-i}(-s)$ allows us to change the sign of $i$ and $j$ (or of $u$ and $v$ ). Hence we reduce to the case in which all suffices are positive.

Thus all Steinberg relations hold in $G$, and it follows that the subgroup $H$ of $G$ generated by the Steinberg generators is isomorphic to $\Omega^{+}(2 n, q)$. By (iii) $H$ contains $U^{\prime}$, so it contains all the $N$-conjugates of $U^{\prime}$, and hence contains $V^{\prime}$. The result follows.

9.1.4. A presentation for $\Omega^{+}(2 n, p)$. We give a presentation for $\Omega^{+}(2 n, p)$ on the generating set $\left\{\sigma, Z, U^{\prime}, V^{\prime}\right\}$. Observe that $N_{1}=\left\langle Z, U^{\prime}, V^{\prime}\right\rangle$, as a subgroup of $\Omega^{+}(2 n, p)$, acts transitively by conjugation on the set of elements $\sigma_{i j}(\epsilon)$, where $|i| \neq|j|$ and $\epsilon= \pm 1$. For 
example, $\sigma_{12}(1)^{U^{\prime 2 V^{\prime}}}=\sigma_{12}(-1)$. The number of symbols of this form is $8 n(n-1)$; but as group elements they are equal in pairs, since $\sigma_{i j}(s)=\sigma_{-j,-i}(-s)$. The centraliser of $\sigma$ in $N_{1}$ is the direct product of the cyclic group of order 4 generated by $Z_{12} U^{\prime}$ with the subgroup of $N_{1}$ that has index $4 n(n-1)$ and centralises $\left\langle e_{1}, f_{1}, e_{2}, f_{2}\right\rangle$.

Theorem 9.5. Let $p$ be an odd prime and let $n \geq 3$. Let $G$ be the group generated by $\left\{\sigma, Z, U^{\prime}, V^{\prime}\right\}$ subject to the relations given below. Then $G$ is isomorphic to $\Omega^{+}(2 n, p)$.

(i) Defining relations for $N_{1}=\left\langle Z, U^{\prime}, V^{\prime}\right\rangle$ as in Theorem 9.1 .

(ii) Relations that state that the elements listed in Theorem 9.3 centralise $\sigma$, but omitting those involving $\Delta$.

(iii) Relations that present $\mathrm{SL}(2, p)$ on $\left\{\sigma, U^{\prime}\right\}$.

(iv) The Steinberg relations (iv) of Theorem 9.4 .

Proof. By (i) we may define the elements $Z_{i j}$ in $G$.

By (ii) we may define $\sigma_{i j}( \pm 1)$ in $G$ for $|i| \neq|j|$ by conjugation, and then define $\sigma_{i j}(s)$ to be $\sigma_{i j}(1)^{s}$, taking $s$ as an integer, since $\sigma_{i j}(1)^{p}=\sigma^{p}=1$ by (iii).

As in the case $e>1,\left\langle\sigma_{12}(1), \sigma_{1,-2}(1), Z, U^{\prime}\right\rangle$ is isomorphic in $G$ to $\Omega^{+}(4, p)$.

The parenthetical Steinberg relations under each of the headings (a) to (d) all follow from one example, as follows. We may assume, by conjugation, that the relation holds for the given suffices with $s=t=1$. In relations (b), (c), (d) and (e), where the RHS is 1 , the case of general $s$ and $t$ follows from the fact that if $[a, b]=1$ and $[a, c]=1$ then $[a, b c]=1$. Relation (a) follows similarly: if $[a, b, a]=[a, b, b]=1$ then $\left[a^{m}, b\right]=\left[a, b^{m}\right]=[a, b]^{m}$.

We thus deduce that all Steinberg relations hold in $G$, and complete the proof arguing as in the case $e>1$.

Observe that $\Delta=\left(\sigma^{\omega-\omega^{2}}\right)^{U^{\prime}} \sigma^{\omega^{-1}}\left(\sigma^{\omega-1}\right)^{U^{\prime}} \sigma^{-1}$.

9.1.5. A presentation for $\mathrm{P} \Omega^{+}(2 n, q)$. The centre of $\Omega^{+}(2 n, q)$ is trivial, unless $q^{n} \equiv 1 \bmod$ 4 , when it has order 2 . If it is non-trivial, then it is generated by $V^{\prime n}$ if $n$ is even, and by $\left(V^{\prime} Z \Delta\right)^{n(q-1) / 4}$ if $n$ is odd. For $\left(V^{\prime} Z \Delta\right)^{n}$ acts on one $n$-dimensional space containing $e_{1}$ by multiplication by $\omega^{-2}$, and on a complementary $n$-dimensional space containing $f_{1}$ by multiplication by $\omega^{2}$.

9.1.6. Standard generators for $\Omega^{+}(2 n, q)$. In Table 2 of [21] the non-trivial standard generators for $\Omega^{+}(2 n, q)$ are labelled $s, t, \delta, s^{\prime}, t^{\prime}, \delta^{\prime}, v$. Observe that $s=Z U^{\prime-1} ; t=\left(\sigma^{-1}\right)^{Z^{V^{\prime}}} ; \delta=$ $\Delta^{\left(Z^{V^{\prime-1}}\right)} ; s^{\prime}=U^{\prime} ; t^{\prime}=\sigma ; \delta^{\prime}=\Delta^{-1}$; and $v=V^{\prime}$. The presentation generator $Z=s s^{\prime}$.

\subsection{Even characteristic.}

9.2.1. Generators and notation. Let $q=2^{e}$. Let $\omega$ be a primitive element of $\operatorname{GF}(q)$.

Let $n>1$. We take a hyperbolic basis $\left(e_{1}, f_{1}, \ldots, e_{n}, f_{n}\right)$ of the natural module, so that, if $Q$ is the quadratic form that is preserved, then $Q\left(e_{i}\right)=Q\left(f_{i}\right)=0$ for all $i$, and the corresponding bilinear form is defined by $e_{i} . f_{i}=1$ and vanishes on all other pairs of basis vectors.

We define the following elements of $\mathrm{SO}^{+}(2 n, q)$, where $1 \leq i, j \leq n$ and $i \neq j$, and $s \in \operatorname{GF}(q)$.

$$
\delta_{i}=\left(e_{i} \mapsto \omega^{-1} e_{i}, f_{i} \mapsto \omega f_{i}\right) \text { if } e>1 ;
$$




$$
\begin{aligned}
& z_{i}=\left(e_{i}, f_{i}\right) \\
& Z_{i j}=z_{i} z_{j} \\
& \sigma_{i j}(s)=\left(e_{i} \mapsto e_{i}+s e_{j}, f_{j} \mapsto f_{j}+s f_{i}\right) \\
& \sigma_{-i, j}(s)=\left(f_{i} \mapsto f_{i}+s e_{j}, f_{j} \mapsto f_{j}+s e_{i}\right)=\sigma_{i j}(s)^{z_{i}} \\
& \sigma_{i,-j}(s)=\left(e_{i} \mapsto e_{i}+s f_{j}, e_{j} \mapsto e_{j}+s f_{i}\right)=\sigma_{i j}(s)^{z_{j}} \\
& \sigma_{-i,-j}(s)=\sigma_{j i}(s)=\sigma_{i j}(s)^{Z_{i j}} ; \\
& U=\left(e_{1}, e_{2}\right)\left(f_{1}, f_{2}\right) ; \\
& V=\left(e_{1}, e_{2}, \ldots, e_{n}\right)\left(f_{1}, f_{2}, \ldots, f_{n}\right)
\end{aligned}
$$

All these elements of $\mathrm{SO}^{+}(2 n, q)$ centralise those basis elements that they are not stated to move. All lie in $\Omega^{+}(2 n, q)$ except for $z_{i}$. Note that $\left\langle\sigma_{12}(1), \delta_{1} \delta_{1}^{-U}, U, V\right\rangle$ is isomorphic to $\operatorname{SL}(n, q)$.

9.2.2. A presentation for $\Omega^{+}(4, q)$. Recall that $\Omega^{+}(4, q)$ is the direct product of two copies of $\mathrm{SL}(2, q)$. One acts on the spaces $\left\langle e_{1}, e_{2}\right\rangle$ and $\left\langle f_{1}, f_{2}\right\rangle$ and is generated by $\left\{\sigma_{12}(1), \delta_{1} \delta_{2}^{-1}, U\right\}$; the other acts on the spaces $\left\langle e_{1}, f_{2}\right\rangle$ and $\left\langle f_{1}, e_{2}\right\rangle$ and is generated by $\left\{\sigma_{1,-2}(1), \delta_{1} \delta_{2}, Z_{12} U\right\}$. The two representations of each copy of $\operatorname{SL}(2, q)$ are linked by the inverse transform automorphism. Conjugating by $z_{2}$ interchanges the generating set of the first of these copies of $\mathrm{SL}(2, q)$ with that of the second, reflecting the fact that $\mathrm{SO}^{+}(4, q)$ is isomorphic to $\mathrm{SL}(2, q) 2$ $C_{2}$. This gives the required presentation on $\left\{\sigma_{12}(1), \delta_{1} \delta_{2}^{-1}, U, \sigma_{1,-2}(1), \delta_{1} \delta_{2}, Z_{12} U\right\}$, omitting $\delta_{1} \delta_{2}^{-1}$ and $\delta_{1} \delta_{2}$ if $e=1$.

9.2.3. A presentation for $\Omega^{+}(2 n, q)$ for $n \geq 3$ and $e>1$. We give a presentation for $\Omega^{+}(2 n, q)$ on the generating set $\left\{\sigma=\sigma_{12}(1), \delta=\delta_{1}, Z=Z_{12}, U, V\right\}$.

Let $N_{1}$ be the subgroup of $\Omega^{+}(2 n, q)$ generated by $\{Z, U, V\}$. It is isomorphic to a subgroup of index 2 in $C_{2}$ ? $S_{n}$.

Theorem 9.6. Let $n \geq 3$. Let $G$ be the group generated by $\{Z, U, V\}$ subject to the relations given below. Then $G$ is isomorphic to $N_{1}$.

(i) Defining relations for $S_{n}$ on $\{U, V\}$.

(ii) If $n>3$ then $\left[Z, U^{V^{2}}\right]=1$.

(iii) If $n>4$ then $\left[Z, V U U^{V}\right]=1$.

(iv) $Z^{2}=1$.

(v) If $n>3$ then $\left[Z, Z^{V^{2}}\right]=1$.

(vi) $Z Z^{V}=Z^{U^{V}}$.

(vii) $[Z, U]=1$.

(viii) $\left[Z, Z^{V}\right]=1$.

Proof. Since, for the stated $n, U^{V^{2}}$ and $V U U^{V}$, as permutations of $\{1,2, \ldots, n\}$, stand for $(3,4)$ and $(3,4, \ldots, n)$, relations (ii) and (iii) allow us to define $Z_{i j}$ in $G$, and (vii) then implies that $Z_{12}=Z_{21}$, so $Z_{i j}=Z_{j i}$ for all $i \neq j$. By (v) and (viii) $Z_{12}$ commutes with $Z_{23}$ and with $Z_{34}$, and hence all $Z_{i j}$ commute with each other, generating an elementary abelian 2-group $A$ by (iv). Finally $Z_{12} Z_{23}=Z_{13}$ by (vi), so $Z_{i j} Z_{j k}=Z_{i k}$ for all distinct $i, j, k$, and $A$ is generated by $\left\{Z_{12}, Z_{23}, \ldots, Z_{n-1, n}\right\}$ and has rank at most $n-1$. But this group has rank $n-1$ in $N_{1}$, and the result follows. 
Let $N=\langle\delta, Z, U, V\rangle \leq \Omega^{+}(2 n, q)$. It is isomorphic to a subgroup of index 2 in $D_{2(q-1)} \prec S_{n}$.

Theorem 9.7. Let $q=2^{e}$ with $e>1$, and let $n \geq 3$. Let $G$ be the group generated by $\{\delta, Z, U, V\}$ subject to the relations given below. Then $G$ is isomorphic to $N$.

(i) Defining relations for $N_{1}=\langle Z, U, V\rangle$ as in Theorem 9.6 .

(ii) $\left[\delta, U^{V}\right]=1$.

(iii) If $n>3$ then $[\delta, V U]=1$.

(iv) $\left[\delta, \delta^{U}\right]=1$.

(v) $\delta^{q-1}=1$

(vi) $\delta^{Z}=\delta^{-1}$.

(vii) $\left[\delta, Z^{V}\right]=1$.

Proof. Since $U^{V}$ and $V U$ stand for $(2,3)$ and $(2,3, \ldots, n)$ respectively, (ii) and (iii) allow us to define $\delta_{i}$ in $G$. Now (iv) implies that $\delta_{1}$ commutes with $\delta_{2}$, and hence the $\delta_{i}$ generate an abelian group $A$, of exponent dividing $q-1$ by (v), and of rank at most $n$. Since $A$ is a group of order $(q-1)^{n}$ in $\Omega^{+}(2 n, q)$ this must also be the case in $G$. In $G$, the subgroup of $N$ generated by the $Z_{i j}$ normalises $A$. This is guaranteed by (vi) and (vii), which assert that $\delta_{1}$ is inverted by $Z_{12}=Z_{21}$ and centralised by $Z_{23}$. So $G / A$ is isomorphic to a subgroup of index 2 in $C_{2}$ ? $S_{n}$. The result follows.

Since $n \geq 3, N$ acts transitively by conjugation on $\left\{\sigma_{i j}(s)|| i|\neq| j \mid, s \in \mathrm{GF}(q)^{\times}\right\}$, a set of size $2 n(n-1)(q-1)$, as follows. Elements of $\langle Z, U, V\rangle$ permute the suffices, acting as signed permutations, where $Z_{i j}$ maps $i$ to $-i$, and $j$ to $-j$, and fixes the other suffices.

Theorem 9.8. Let $q=2^{e}$ with $e>1$, and let $n \geq 3$. The centraliser of $\sigma=\sigma_{12}(1)$ in $N$ has index $2 n(n-1)(q-1)$ in $N$ and is generated by the following elements:

(i) $U^{V^{2}}$ if $n>3$,

(ii) $V U U^{V}$ if $n>4$;

(iii) $Z^{V^{2}}$ if $n>3$;

(iv) $\delta^{V^{2}}$;

(v) $Z U$;

(vi) $\delta \delta^{U}$.

Proof. The centraliser of $\sigma$ in $N$ is the direct product of the centraliser in $N$ of $\left\langle e_{1}, f_{1}, e_{2}, f_{2}\right\rangle$, which has order $(q-1)^{n-2} 2^{n-3}(n-2)$ !, with a copy of $D_{2(q-1)}$, namely $\left\langle\delta_{1} \delta_{2}, Z_{12} U\right\rangle$.

Theorem 9.9. Let $q=2^{e}$ with $e>1$, and let $n \geq 3$. Let $G$ be the group generated by $\{\sigma, \delta, Z, U, V\}$ subject to the relations given below. Then $G$ is isomorphic to $\Omega^{+}(2 n, q)$.

(i) Defining relations for $N=\langle\delta, Z, U, V\rangle$ as in Theorem 9.7 .

(ii) Relations that state that the elements listed in Theorem 9.8 centralise $\sigma$.

(iii) Relations that present $\operatorname{SL}(2, q)$ on $\{\sigma, \Delta, U\}$, where $\Delta=[U, \delta]=\delta_{1} \delta_{2}^{-1}$, but omitting the relation $\Delta^{q-1}=1$.

(iv) The following instances of Steinberg relations:

(a) $\left[\sigma, \sigma^{V}\right]=\sigma^{V U} ; \quad\left(\left[\sigma_{i j}(s), \sigma_{j k}(t)\right]=\sigma_{i k}(s t)\right)$;

(b) $\left[\sigma, \sigma^{V U}\right]=1 ; \quad\left(\left[\sigma_{i j}(s), \sigma_{i k}(t)\right]=1\right)$;

(c) $\left[\sigma, \sigma^{W}\right]=1$, where $W=U^{V U} ; \quad\left(\left[\sigma_{i j}(s), \sigma_{k j}(t)\right]=1\right)$; 
(d) if $n>3$ then $\left[\sigma, \sigma^{V^{2}}\right]=1 ; \quad\left(\left[\sigma_{i j}(s), \sigma_{k l}(t)\right]=1\right)$;

(e) $\left[\sigma, \sigma^{Z^{V}}\right]=1 ; \quad\left(\left[\sigma_{i j}(s), \sigma_{i,-j}(t)\right]=1\right)$;

(f) if $n=4$ then $\left[\sigma, \sigma^{Z^{V} V^{2}}\right]=1 ; \quad\left(\left[\sigma_{i j}(s), \sigma_{k,-l}(t)\right]=1\right)$.

The given relations are instances of the more general relations in parentheses, with $s=t=1$ and $(i, j, k, l)=(1,2,3,4)$. In the parenthetical version, $i, j, k, l$ are distinct and positive.

Proof. Relations (i) imply that $\left(\delta_{1} \delta_{2}\right)^{q-1}=1$, which supplies the relation omitted from (iii).

By (i) we may define the elements $Z_{i j}$ and $\delta_{i}$ in $G$. Since $N$ acts transitively, in $\Omega^{+}(2 n, q)$, by conjugation on the set of all root elements $\sigma_{i j}(s)$, where $s \neq 0$, we may define $\sigma_{i j}(s)$ in $G$ by the same conjugation, by (ii).

Recall that $\mathrm{SO}^{+}(4, q)$ is isomorphic to $\mathrm{SL}(2, q) \imath C_{2}$. Here $\left\langle\sigma_{12}, \delta_{1} \delta_{2}^{-1}, U\right\rangle$ is one copy of $\operatorname{SL}(2, q)$, and $\left\langle\sigma_{1,-2}, \delta_{1} \delta_{2}, Z_{12} U\right\rangle$ is another. The first of these subgroups in $G$ is isomorphic to $\operatorname{SL}(2, q)$ by (iii), and, since $Z_{12} U=U^{Z_{23}}$, so is the second, by conjugation with $Z_{23}$. By (iv)(e), (i) and (ii), these subgroups commute with each other. It follows that, in $G$, the subgroup $\left\langle\sigma_{ \pm 1, \pm 2}(1), \delta, Z, Z^{V}, U\right\rangle$ is isomorphic to $\mathrm{SO}^{+}(4, q)$, where $Z^{V}$ acts as a wreathing element. Thus all Steinberg relations that involve only two suffices, with various signs, hold in $G$.

The parenthetical Steinberg relations under each of the headings (a) to (d) all follow from one example, as in the case of odd $q$, the suffices all being positive.

All remaining Steinberg relations involving negative suffices may be deduced as in the case of odd $q$.

The proof that $G$ is generated by the Steinberg generators follows as before.

Recall that $\Omega^{+}(2 n, q)$ is simple for even $q$.

9.2.4. A presentation for $\Omega^{+}(2 n, 2)$ for $n \geq 3$.

Theorem 9.10. Let $n \geq 3$. Let $G$ be the group generated by $\{\sigma, Z, U, V\}$ subject to the relations given below. Then $G$ is isomorphic to $\Omega^{+}(2 n, 2)$.

(i) Defining relations for $N_{1}=\langle Z, U, V\rangle$ as in Theorem 9.6 .

(ii) Relations that state that the elements listed in Theorem 9.8 centralise $\sigma$, but omitting those involving $\delta$.

(iii) Relations that present $\operatorname{SL}(2,2)$ on $\{\sigma, U\}$.

(iv) The Steinberg relations (iv) of Theorem 9.9 .

Proof. By (i) we may define the elements $Z_{i j}$ in $G$. Since $N_{1}$ acts transitively, in $\Omega^{+}(2 n, 2)$, by conjugation on the set of $2 n(n-1)$ root elements $\sigma_{ \pm i, \pm j}(1)$, we may define $\sigma_{i j}(1)$ in $G$ by the same conjugation, by (ii).

As in the case $e>1,\left\langle\sigma_{12}(1), U, \sigma_{1,-2}(1), Z\right\rangle$ is isomorphic in $G$ to $\Omega^{+}(4,2)$.

The parenthetical Steinberg relations, under each of the headings (a) to (e), all follow from one example, by conjugation. We complete the proof arguing as in the case $e>1$.

Observe that $\delta=1$. 
9.2.5. Standard generators for $\Omega^{+}\left(2 n, 2^{e}\right)$. In Table 1 of [10] the non-trivial standard generators for $\Omega^{+}\left(2 n, 2^{e}\right)$ are labelled $s, t, \delta, u, x, y, v$. Observe that $s=Z U ; t=\left(\sigma^{-1}\right)^{Z^{V}} ; u=$ $U ; x=\sigma ; y=\left(\delta \delta^{Z U}\right)^{-1} ; v=V$; and the standard generator $\delta=\delta^{-1}\left(\delta^{-1}\right)^{U}$. The presentation generators $\delta=(\delta y)^{(q-2) / 2}$ and $Z=s u$.

\section{A presentation For $\Omega(2 n+1, q)$}

We may assume that $q$ is odd, since $\Omega(2 n+1, q)$ is isomorphic to $\operatorname{Sp}(2 n, q)$ if $q$ is even. Since $\Omega(3, q)$ is isomorphic to $\operatorname{PSL}(2, q)$ for odd $q$, we assume that $n>1$.

10.1. Generators and notation. Let $q=p^{e}$ for an odd prime $p$. Let $\omega$ be a primitive element of $\operatorname{GF}(q)$.

Let $n>1$. We take a basis $\left(e_{1}, f_{1}, e_{2}, f_{2}, \ldots, e_{n}, f_{n}, w\right)$ of the natural module, with symmetric bilinear form defined by $e_{i} \cdot f_{i}=f_{i} \cdot e_{i}=1$ for all $i$, and $w \cdot w=-2$, and the form vanishes on all other pairs of basis vectors. Note that, with our standard generators, we assume that $w \cdot w=-1 / 2$; we have made this change for compatibility with our treatment of $\Omega^{-}(2 n, q)$ for odd $q$.

We define the following elements of $\mathrm{SO}(2 n+1, q)$, where $1 \leq i, j \leq n$ and $i \neq j$, and $s \in \mathrm{GF}(q)$.

$$
\begin{aligned}
& \delta_{i}=\left(e_{i} \mapsto \omega^{-1} e_{i}, f_{i} \mapsto \omega f_{i}\right) \text { if } e>1 ; \\
& \Delta_{i j}=\delta_{i} \delta_{j}^{-1} ; \\
& \Delta_{-i, j}=\delta_{i}^{-1} \delta_{j}^{-1} ; \\
& \Delta_{i,-j}=\delta_{i} \delta_{j} ; \\
& \Delta_{-i,-j}=\delta_{i}^{-1} \delta_{j} ; \\
& z_{i}=\left(e_{i}, f_{i}\right)(w)^{-} ; \\
& \sigma_{i j}(s)=\left(e_{i} \mapsto e_{i}+s e_{j}, f_{j} \mapsto f_{j}-s f_{i}\right) ; \\
& \sigma_{i,-j}(s)=\left(e_{i} \mapsto e_{i}+s f_{j}, e_{j} \mapsto e_{j}-s f_{i}\right)=\sigma_{i j}(s)^{z_{j}} ; \\
& \sigma_{-i, j}(s)=\left(f_{i} \mapsto f_{i}+s e_{j}, f_{j} \mapsto f_{j}-s e_{i}\right)=\sigma_{i j}(s)^{z_{i}} ; \\
& \sigma_{-i,-j}(s)=\sigma_{j i}(-s)=\sigma_{i j}(s)^{z_{i} z_{j}} ; \\
& \tau_{i}(s)=\left(e_{i} \mapsto e_{i}+s^{2} f_{i}+s w, w \mapsto w+2 s f_{i}\right) ; \\
& \tau_{-i}(s)=\left(f_{i} \mapsto f_{i}+s^{2} e_{i}-s w, w \mapsto w-2 s e_{i}\right)=\tau_{i}(s)^{z_{i}} ; \\
& U=\left(e_{1}, e_{2}\right)\left(f_{1}, f_{2}\right) ; \\
& U^{\prime}=\left(e_{1}, e_{2}\right)^{-}\left(f_{1}, f_{2}\right)^{-} ; \\
& V=\left(e_{1}, e_{2}, \ldots, e_{n}\right)\left(f_{1}, f_{2}, \ldots, f_{n}\right) ; \\
& V^{\prime}=\left(e_{1}, e_{2}, \ldots, e_{n}\right)^{\epsilon_{n}}\left(f_{1}, f_{2}, \ldots, f_{n}\right)^{\epsilon_{n}} .
\end{aligned}
$$

All these elements of $\mathrm{SO}(2 n+1, q)$ centralise those basis elements that they are not stated to move. All lie in $\Omega(2 n+1, q)$ except for $\delta_{i}$, and $U$ if $q \equiv 3 \bmod 4$, and $V$ if $n$ is even and $q \equiv 3 \bmod 4$.

If $n>2$ then $\left\langle\sigma_{12}(1), \Delta_{12}, z_{1} z_{1}^{U^{\prime}}, U^{\prime}, V^{\prime}\right\}$ acts as the natural copy of $\Omega^{+}(2 n, q)$ on $\left\langle e_{1}, f_{1}, \ldots, e_{n}, f_{n}\right\rangle$.

10.2. A presentation for $\Omega(2 n+1, q)$ for $n \geq 2$ and $e>1$. We give a presentation for $\Omega(2 n+1, q)$ on the generating set $\left\{\sigma=\sigma_{12}(1), \tau=\tau_{1}(1), \Delta=\Delta_{12}, z=z_{1}, U^{\prime}, V^{\prime}\right\}$, omitting $V^{\prime}$ if $n=2$.

Let $N_{1}$ be the subgroup of $\Omega(2 n+1, q)$ generated by $\left\{z, U^{\prime}, V^{\prime}\right\}$, omitting $V^{\prime}$ if $n=2$. This is an extension of an elementary abelian 2-group $A$ of rank $2 n-1$ by a copy of 
$S_{n}$. Here $A$ is the direct product of a subgroup of rank $n-1$, generated by $U^{\prime 2}$ and its conjugates, with a second subgroup of rank $n$ generated by $z$ and its conjugates.

Theorem 10.1. Let $n \geq 2$. Let $G$ be the group generated by $\left\{z, U^{\prime}, V^{\prime}\right\}$ subject to the relations given below. Then $G$ is isomorphic to $N_{1}$.

(i) Defining relations for $\left\langle U^{\prime}, V^{\prime}\right\rangle$ as in Theorems 2.2 and 2.3, omitting $V^{\prime}$ if $n=2$.

(ii) If $n>2$ then $\left[z, U^{\prime V^{\prime}}\right]=1$.

(iii) If $n>3$ then $\left[z, V^{\prime} U^{\prime-1}\right]=1$.

(iv) $z^{2}=1$.

(v) $\left[z, U^{\prime 2}\right]=1$.

(vi) $\left[z, z^{U^{\prime}}\right]=1$.

Proof. Relations (ii) and (iii) state that $z$ is centralised by the subgroup of $\left\langle U^{\prime}, V^{\prime}\right\rangle$ that centralises 1 as signed permutations, since $U^{\prime V^{\prime}}$ and $V^{\prime} U^{\prime-1}$ stand for $(2,3)^{-}$and $(2,3, \ldots, n)^{-\epsilon_{n}}$ respectively as signed permutations.

These relations, together with (v), also imply that $z$ is centralised by all $\left\langle U^{\prime}, V^{\prime}\right\rangle$ conjugates of $U^{\prime 2}$, so we may label the conjugates of $z$ as $z_{i}$, where $1 \leq i \leq n$. Relation (vi) states that $z=z_{1}$ commutes with $z_{2}$; hence, by conjugation, all $z_{i}$ commute and, by (iv), generate an elementary abelian 2-group. Thus $G$ is a split extension of a normal subgroup of order at most $2^{n}$ by the group $\left\langle U^{\prime}, V^{\prime}\right\rangle$ of order $2^{n-1} n$ !, by (i). But $N_{1}$, as a subgroup of $\Omega(2 n+1, q)$, has order $2^{2 n-1} n$ !, and the result follows.

Let $N=\left\langle\Delta, z, U^{\prime}, V^{\prime}\right\rangle \leq \Omega(2 n+1, q)$, omitting $V^{\prime}$ if $n=2$. As with $\Omega^{+}(2 n, q)$, the normal subgroup of $N$ generated as normal subgroup by $\Delta$, and as subgroup by $\left\{\Delta_{ \pm i, \pm j}|| i|\neq| j \mid\right\}$, has order $(q-1)^{n} / 2$. Since this normal subgroup contains $U^{\prime 2}$, it follows that $N$ has order $(q-1)^{n} 2^{n-1} n$ !.

Theorem 10.2. Let $q=p^{e}$ for an odd prime $p$ and $e>1$, and let $n \geq 2$. Let $G$ be the group generated by $\left\{\Delta, z, U^{\prime}, V^{\prime}\right\}$, omitting $V^{\prime}$ if $n=2$, subject to the relations given below. Then $G$ is isomorphic to $N$.

(i) Defining relations for $N_{1}=\left\langle z, U^{\prime}, V^{\prime}\right\rangle$ as in Theorem 10.1, omitting $V^{\prime}$ if $n=2$.

(ii) If $n>3$ then $\left[\Delta, U^{\prime V^{\prime 2}}\right]=1$.

(iii) If $n>4$ then $\left[\Delta, V^{\prime} U^{\prime} U^{\prime V^{\prime}}\right]=1$.

(iv) If $n>2$ then $\left[\Delta, z^{V^{\prime 2}}\right]=1$.

(v) $\Delta^{U^{\prime}}=\Delta^{-1}$, and if $n>2$ then $\left[\Delta, U^{\prime 2 V}\right]=1$.

(vi) $\Delta^{(q-1) / 2}=U^{\prime 2}$.

(vii) If $n>2$ then $\Delta \Delta^{V^{\prime}}=\Delta^{V^{\prime} U^{\prime}}$.

(viii) If $n>2$ then $\left[\Delta, \Delta^{V^{\prime}}\right]=1$.

(ix) If $n>3$ then $\left[\Delta, \Delta^{V^{\prime 2}}\right]=1$.

(x) $\Delta^{z z^{U^{\prime}}}=\Delta^{-1}$.

(xi) If $n=2$ then $\left[\Delta, \Delta^{z}\right]=1$.

Proof. Suppose first that $n \geq 3$. This presentation is very similar to that for $N$ in Theorem 9.2. The subgroups $N$ and $N_{1}$ of $\Omega^{+}(2 n, q)$ have index 2 in the corresponding subgroups of $\Omega(2 n+1, q)$, the generator $Z=Z_{12}$ being replaced by $z=z_{1}$. The presentation for $N$ given in this theorem differs from the presentation in Theorem 9.2 in items (i) and (iv). Item 
(i) gives a presentation for the enlarged version of $N_{1}$ that results from replacing $Z$ by $z$. Item (iv) asserts that $\Delta$ is centralised by $z_{3}$, and hence, by conjugation, by $z_{3}, z_{4}, \ldots, z_{n}$, rather than by $Z_{34}, Z_{45}, \ldots, Z_{n-1, n}$ in the case of $\Omega^{+}(2 n, q)$. This reflects the fact that the centraliser of $\Delta$ in $N_{1}$ is twice as large in the case of $\Omega(2 n+1, q)$, since both groups $N_{1}$ conjugate transitively the same set of elements $\Delta_{i j}$. The proof now follows that of Theorem 9.2 .

The proof for $n=2$ is easy.

The centraliser of $\sigma=\sigma_{12}(1)$ in $N$ is the direct product of the subgroup of $N$ that centralises $\left\langle e_{1}, f_{1}, e_{2}, f_{2}\right\rangle$ with the copy of $Q_{2(q-1)}$ generated by $\left\{\Delta_{1,-2}, U^{\prime z}\right\}$. If $n=2$ then the centraliser is $Q_{2(q-1)}$. If $n>2$ then the centraliser has order $(q-1)^{n-1} 2^{n-2}(n-2)$ !, and index $2(q-1) n(n-1)$ in $N$; this corresponds to the fact that $N$ acts transitively by conjugation on the set of elements $\sigma_{ \pm i, \pm j}(s)$, where $1 \leq i \neq j \leq n$ and $s \in \operatorname{GF}(q)^{\times}$, and these are equal in pairs.

Theorem 10.3. The centraliser of $\tau=\tau_{1}(1)$ in $N$ has index $2(q-1) n$ in $N$ and is generated by the following elements:

(i) $U^{\prime V^{\prime}}$ if $n>2$;

(ii) $V^{\prime} U^{\prime-1}$ if $n>3$;

(iii) $\Delta^{V^{\prime}}$ if $n>2$;

(iv) $U^{\prime 2} z^{U^{\prime}}$;

(v) $\Delta \Delta^{z}$ if $n=2$.

Proof. The centraliser of $\tau$ in $N$ is the direct product of the subgroup of $N$ that centralises $\left\langle e_{1}, f_{1}, w\right\rangle$ with $\left\langle U^{\prime 2} z_{2}\right\rangle$, and so has order $(q-1)^{n-1} 2^{n-2}(n-1)$ !, and index $2(q-1) n$ in $N$. This corresponds to the fact that $N$ acts transitively by conjugation on the $2(q-1) n$ root elements $\tau_{ \pm i}(s)$, where $s \in \mathrm{GF}(q)^{\times}$.

Theorem 10.4. Let $q=p^{e}$ for an odd prime $p$ and $e>1$, and let $n \geq 2$. Let $G$ be the group generated by $\left\{\sigma, \tau, \Delta, z, U^{\prime}, V^{\prime}\right\}$, omitting $V^{\prime}$ if $n=2$, subject to the relations given below. Then $G$ is isomorphic to $\Omega(2 n+1, q)$.

(i) Defining relations for $N=\left\langle\Delta, z, U^{\prime}, V^{\prime}\right\rangle$, omitting $V$ if $n=2$, as in Theorem 10.2 , but omitting relation (vi) of that theorem.

(ii) $\left[\sigma, \Delta^{z^{U^{\prime}}}\right]=1$.

(iii) Relations that state that the elements listed in Theorem 10.3 centralise $\tau$.

(iv) Relations that present $\operatorname{SL}(2, q)$ on $\left\{\sigma, \Delta, U^{\prime}\right\}$.

(v) Relations that present $\operatorname{PSL}(2, q)$ on $\left\{\tau, \Delta \Delta^{z^{U^{\prime}}}, z\right\}$.

(vi) The following instances of Steinberg relations:

(a) if $n>2$ then $\left[\sigma, \sigma^{V^{\prime}}\right]=\sigma^{V^{\prime} U^{\prime-1}} ; \quad\left(\left[\sigma_{i j}(s), \sigma_{j k}(t)\right]=\sigma_{i k}(s t)\right)$;

(b) if $n>2$ then $\left[\sigma, \sigma^{V^{\prime} U^{\prime-1}}\right]=1 ; \quad\left(\left[\sigma_{i j}(s), \sigma_{i k}(t)\right]=1\right)$;

(c) if $n>2$ then $\left[\sigma, \sigma^{W}\right]=1$, where $W=U^{\prime V^{\prime} U^{\prime-1}} ; \quad\left(\left[\sigma_{i j}(s), \sigma_{k j}(t)\right]=1\right)$;

(d) if $n>3$ then $\left[\sigma, \sigma^{V^{\prime 2}}\right]=1 ; \quad\left(\left[\sigma_{i j}(s), \sigma_{k l}(t)\right]=1\right)$;

(e) $\left[\sigma, \sigma^{z^{U^{\prime}}}\right]=1 ; \quad\left(\left[\sigma_{i j}(s), \sigma_{i,-j}(t)\right]=1\right)$;

(f) $\left[\tau, \tau^{U^{\prime}}\right]=\sigma^{2 z^{U^{\prime}}} ; \quad\left(\left[\tau_{i}(s), \tau_{j}(t)\right]=\sigma_{i,-j}(2 s t)\right)$;

(g) $[\sigma, \tau]=1 ; \quad\left(\left[\sigma_{i j}(s), \tau_{i}(t)\right]=1\right)$; 
(h) $\left[\sigma^{z}, \tau\right]=\sigma \tau^{z U^{\prime}} ; \quad\left(\left[\sigma_{-i, j}(s), \tau_{i}(t)\right]=\sigma_{i j}\left(s t^{2}\right) \tau_{-j}(s t)\right)$;

(j) if $n>2$ then $\left[\sigma, \tau^{V^{\prime 2}}\right]=1 ; \quad\left(\left[\sigma_{i j}(s), \tau_{k}(t)\right]=1\right)$.

The given relations are instances of the more general relations in parentheses, with $s=t=1$, and $(i, j, k, l)=(1,2,3,4)$. In the parenthetical version, $i, j, k, l$ are distinct and positive.

Proof. Relation (vi) of Theorem 10.2, omitted from (i) of this theorem, is provided by (iv). Since, in the matrix group, $N$ acts transitively by conjugation on $\left\{\tau_{ \pm i}(s) \mid 1 \leq i \leq\right.$ $\left.n, s \in \mathrm{GF}(q)^{\times}\right\}$, we may define the elements $\tau_{ \pm i}(s)$ in $G$, using (i) and (iii).

It follows from (f) that $\sigma^{2}=\left[\tau_{1}(-1), \tau_{-2}(1)\right]$. So $\sigma^{2}$ is centralised by the subgroup $A$ of $N$ that centralises $\left\langle e_{1}, f_{1}, e_{2}, f_{2}\right\rangle$, and also by $U^{\prime z}$. Also, $\sigma$ has odd order by (iv); so, since $\Delta_{1,-2}=\Delta^{z^{U^{\prime}}}$, relations (ii) and (iv) complete a generating set for the centraliser of $\sigma$ in $N$. Thus we may define the elements $\sigma_{i j}(s)$ for all $i$ and $j$, positive or negative, in $G$.

The Steinberg relations for the individual root groups may be deduced from (iv) and (v). We must show that the Steinberg relations that give the commutator of two long root elements, that is, of two elements of the form $\sigma_{i j}(s)$, are all consequences of (a) to (e). These relations correspond precisely to the Steinberg relations (iv)(a)-(iv)(e) of Theorem 9.4, except for an insignificant change to (iv)(e). Since the torus normaliser $N$ in the case of $\Omega(2 n+1, q)$ contains the corresponding torus normaliser in $\Omega^{+}(2 n, q)$ as a subgroup of index 2, the fact that these five instances of Steinberg relations in $\Omega(2 n+1, q)$ suffice to imply all Steinberg relations that give the commutator of two long root elements follows $a$ fortiori from Theorem 9.4. Clearly the Steinberg relations that give the commutator of two short root elements all follow, by conjugation in $N$, from the one instance (f). Similarly the Steinberg relations that give the commutator of a long and a short root element may be deduced from the instances $(\mathrm{g}),(\mathrm{h})$ and $(\mathrm{j})$.

Thus the subgroup $H$ of $G$ generated by the Steinberg generators is isomorphic to $\Omega(2 n+1, q)$; that $U^{\prime}$ and $V^{\prime}$ lie in $H$, which is thus the whole of $G$, follows by the now familiar argument.

Recall that $\Omega^{+}(2 n+1, q)$ is simple.

\subsection{A presentation for $\Omega(2 n+1, p)$ for $n \geq 2$.}

Theorem 10.5. Let $p$ be an odd prime and let $n \geq 2$. Let $G$ be the group generated by $\left\{\sigma, \tau, z, U^{\prime}, V^{\prime}\right\}$, omitting $V^{\prime}$ if $n=2$, subject to the relations given below. Then $G$ is isomorphic to $\Omega(2 n+1, p)$.

(i) Defining relations for $N_{1}=\left\langle z, U^{\prime}, V^{\prime}\right\rangle$ as in Theorem 10.1, omitting $V^{\prime}$ if $n=2$.

(ii) Relations that state that the elements listed in Theorem 10.3 centralise $\tau$, but omitting those involving $\Delta$.

(iii) Relations that present $\mathrm{SL}(2, p)$ on $\left\{\sigma, U^{\prime}\right\}$.

(iv) Relations that present $\operatorname{PSL}(2, p)$ on $\{\tau, z\}$.

(v) The Steinberg relations (vi) of Theorem 10.4.

(vi) If $n=p=3$ then $\left[\tau, \sigma^{V^{\prime}}\right]=1$.

Proof. By (i) we may define the elements $z_{i}$ in $G$.

If $n>2$ then $N_{1}$ acts transitively by conjugation on $\left\{\sigma_{ \pm i, \pm j}( \pm 1) \mid 1 \leq i \neq j \leq n\right\}$; the $8 n(n-1)$ symbols are equal, as matrices, in pairs. Since $N_{1}$ has order $2^{2 n-1} n$ !, the 
centraliser in $N_{1}$ of $\sigma$ has order $2^{2 n-3}(n-2)$ !. Thus this centraliser is generated by the subgroup of $N_{1}$ that centralises $\left\langle e_{1}, f_{1}, e_{2}, f_{2}\right\rangle$, which has order $2^{2 n-5}(n-2)$ ! as $n>2$, together with $U^{\prime 2}$ and $U^{\prime z_{1}}$. It follows, from item (ii) of this theorem and the Steinberg relation (vi)(f) of Theorem 10.4, that these elements of $N_{1}$ centralise $\sigma$ in $G$. Thus the elements $\sigma_{i j}( \pm 1)$ may be defined in $G$ for all $i$ and $j$, positive or negative, and hence all elements $\sigma_{i j}(s)$ for $s$ in $\operatorname{GF}(p)$.

Now let $n=2$. By (ii), we may define the elements $\tau_{i}(s)$ in $G$. Moreover $N_{1}$, as matrices, permutes the 8 elements $\left\{\sigma_{ \pm i, \pm j}( \pm 1) \mid 1 \leq i \neq j \leq 2\right\}$ in 2 orbits of size 4 . Since $N_{1}$ has order 16 , the centraliser of $\sigma$ in $N_{1}$ has order 4 and is generated by $\left\{U^{\prime 2}, U^{\prime z_{1}}\right\}$. As in the case $n>2$ these elements centralise $\sigma$ as elements of $G$, and so we may now define the elements $\sigma_{i j}(s)$ in this group.

The general cases of the Steinberg relations now follow easily from the cases given in the presentation.

We complete the proof arguing as in the case $e>1$.

Relation (vi) is needed because the Steinberg presentation gives the universal Chevalley group, and $\Omega(7,3)$ has a Schur multiplier of order 6 . The involution in the multiplier is killed by our other relations, and (vi) is needed to kill the remaining 3-cycle. We prove correctness of the presentation for this group by coset enumeration.

Observe that $\Delta=\left(\sigma^{\omega-\omega^{2}}\right)^{U^{\prime}} \sigma^{\omega^{-1}}\left(\sigma^{\omega-1}\right)^{U^{\prime}} \sigma^{-1}$.

10.4. Standard generators for $\Omega(2 n+1, q)$. In Table 2 of 21 the standard generators for $\Omega(2 n+1, q)$ are labelled $s, t, \delta, u, v$. Observe that $s=z^{V^{\prime-1}} ; \delta=\left[z, \Delta^{-1}\right]^{V^{\prime-1}} ; u=$ $U^{\prime} ; v=V^{\prime}$; if $d \equiv 3 \bmod 4$ then $t=(\tau)^{V^{\prime-1}}$, else $t=\left(\tau^{-1}\right)^{V^{\prime-1}}$.

The presentation generator $\sigma=\left[\tau^{z U^{\prime}}, \tau^{(p+1) / 2}\right]$. If $q \equiv 3 \bmod 4$ then $\Delta=U^{\prime 2}\left(\left[\delta^{V^{\prime}}, U^{\prime}\right]\right)^{(q+1) / 4}$. Also $\Delta^{2}=\left[\delta^{V^{\prime}}, U^{\prime}\right]$. If $q \neq 9$, then $\Delta \in\left\langle\Delta^{2}, \sigma, U^{\prime}\right\rangle \cong \mathrm{SL}(2, q)$. In particular,

$$
\Delta=\prod_{i=0}^{e-1} \sigma^{c_{i} \Delta^{2 i} U^{\prime}} \Delta^{2} \sigma^{U^{\prime}} \prod_{i=0}^{e-1} \sigma^{b_{i} \Delta^{2 i} \sigma^{U^{\prime}}} \prod_{i=0}^{e-1} \sigma^{a_{i} \Delta^{2 i}}
$$

where $\sum_{i=0}^{e-1} a_{i} \omega^{4 i}=-\omega^{-1}+1$, and $\sum_{i=0}^{e-1} b_{i} \omega^{4 i}=1-\omega$, and $\sum_{i=0}^{e-1} c_{i} \omega^{4 i}=-\omega^{3}$. If $q=9$, then $\Delta \in\left\langle\Delta^{2}, z, U^{\prime}, \tau\right\rangle$, and this group is isomorphic to $\operatorname{PSp}(4,9)$, the natural module for $\Omega(5,9)$ being isomorphic to an irreducible constituent of the exterior square of the natural module for $\operatorname{Sp}(4,9)$; we record $\Delta$ as an explicit fixed word in these generators.

\section{A PRESEntation FOR $\Omega^{-}(2 n, q)$ FOR $n>1$}

Since $\Omega^{-}(4, q) \cong \operatorname{PSL}\left(2, q^{2}\right)$, we assume that $n>2$.

\subsection{Odd characteristic.}

11.1.1. Generators and notation. Let $q=p^{e}$ for an odd prime $p$. Let $\omega$ be a primitive element of $\operatorname{GF}\left(q^{2}\right)$, and let $\omega_{0}=\omega^{q+1}$, so $\omega_{0}$ is a primitive element of $\operatorname{GF}(q)$. Define $\psi=\omega^{(q+1) / 2}$.

Let $n>2$. We take a basis $\left(e_{1}, f_{1}, e_{2}, f_{2}, \ldots, e_{n-1}, f_{n-1}, w_{1}, w_{2}\right)$ of the natural module, with orthogonal bilinear form defined by $e_{i} \cdot f_{i}=f_{i} \cdot e_{i}=1, w_{1} \cdot w_{1}=-2, w_{2} \cdot w_{2}=2 \omega_{0}$, and the form vanishes on all other pairs of basis elements. This form is of type -. 
We define the following elements of $\mathrm{SO}^{-}(2 n, q)$, where $1 \leq i, j \leq n-1$ and $i \neq j$, and $s \in \mathrm{GF}(q)$, and $\alpha \in \mathrm{GF}\left(q^{2}\right)$.

$\delta_{i}=\left(e_{i} \mapsto \omega_{0}^{-1} e_{i}, f_{i} \mapsto \omega_{0} f_{i}, w_{1} \mapsto A w_{1}-C w_{2}, w_{2} \mapsto-B w_{1}+A w_{2}\right)$, where

$$
\begin{aligned}
& A=\left(\omega^{q-1}+\omega^{1-q}\right) / 2 \\
& B=\psi\left(\omega^{1-q}-\omega^{q-1}\right) / 2 \\
& C=\psi^{-1}\left(\omega^{1-q}-\omega^{q-1}\right) / 2 ;
\end{aligned}
$$

$$
\begin{aligned}
& \Delta_{i,-j}=\delta_{i} \delta_{j}^{-1} \\
& z_{i}=\left(e_{i}, f_{i}\right)\left(w_{1}\right)^{-} ; \\
& \sigma_{i j}(s)=\left(e_{i} \mapsto e_{i}+s e_{j}, f_{j} \mapsto f_{j}-s f_{i}\right) ; \\
& \sigma_{i,-j}(s)=\left(e_{i} \mapsto e_{i}+s f_{j}, e_{j} \mapsto e_{j}-s f_{i}\right)=\sigma_{i j}^{z_{j}} ; \\
& \sigma_{-i, j}(s)=\left(f_{i} \mapsto f_{i}+s e_{j}, f_{j} \mapsto f_{j}-s e_{i}\right)=\sigma_{i j}^{z_{i}} ; \\
& \sigma_{-i,-j}(s)=\sigma_{j i}(-s)=\sigma_{i j}(s)^{z_{i} z_{j}} ; \\
& \tau_{i}(\alpha)=\left(e_{i} \mapsto e_{i}+\alpha^{q+1} f_{i}+\frac{1}{2}\left(\alpha^{q}+\alpha\right) w_{1}+\frac{1}{2} \psi^{-1}\left(\alpha^{q}-\alpha\right) w_{2} ;\right. \\
& \left.\quad w_{1} \mapsto w_{1}+\left(\alpha+\alpha^{q}\right) f_{i}, w_{2} \mapsto w_{2}+\psi\left(\alpha-\alpha^{q}\right) f_{i}\right) ; \\
& \tau_{-i}(\alpha)=\left(f_{i} \mapsto f_{i}+\alpha^{q+1} e_{i}-\frac{1}{2}\left(\alpha^{q}+\alpha\right) w_{1}+\frac{1}{2} \psi^{-1}\left(\alpha^{q}-\alpha\right) w_{2},\right. \\
& \left.\quad w_{1} \mapsto w_{1}-\left(\alpha+\alpha^{q}\right) e_{i}, w_{2} \mapsto w_{2}+\psi\left(\alpha-\alpha^{q}\right) e_{i}\right)=\tau_{i}(\alpha)^{z_{i}} ; \\
& U=\left(e_{1}, e_{2}\right)\left(f_{1}, f_{2}\right) ; \\
& U^{\prime}=\left(e_{1}, e_{2}\right)^{-}\left(f_{1}, f_{2}\right)^{-} ; \\
& V=\left(e_{1}, e_{2}, \ldots, e_{n-1}\right)\left(f_{1}, f_{2}, \ldots, f_{n-1}\right) ; \\
& V^{\prime}=\left(e_{1}, e_{2}, \ldots, e_{n-1}\right)^{\epsilon_{n-1}}\left(f_{1}, f_{2}, \ldots, f_{n-1}\right)^{\epsilon_{n-1}}
\end{aligned}
$$

All these elements of $\mathrm{SO}^{-}(2 n, q)$ centralise those basis elements that they are not stated to move. All lie in $\Omega^{-}(2 n, q)$ except for $U$ if $q \equiv 3 \bmod 4$, and $V$ if $n$ is odd and $q \equiv 3 \bmod 4$.

All those elements of $\Omega^{-}(2 n, q)$ that fix $w_{2}$ are defined as the corresponding elements of $\Omega(2 n-1, q)$. In particular, $\tau_{i}(s)$ for $s \in \mathrm{GF}(q)$, and its conjugates under $\delta_{i} \delta_{j}^{-1}$ for $i \neq j$, are the same in $\Omega(2 n-1, q)$ and in $\Omega^{-}(2 n, q)$.

We give a presentation for $\Omega^{-}(2 n, q)$ on the generating set $\left\{\sigma=\sigma_{12}(1), \tau=\tau_{1}(1), \delta=\right.$ $\left.\delta_{1}, z=z_{1}, U^{\prime}, V^{\prime}\right\}$, omitting $V^{\prime}$ if $n=3$.

11.1.2. A presentation for $\Omega^{-}(6, q)$. We exploit the isomorphism between $\Omega^{-}(6, q)$ and $\mathrm{SU}(4, q) /\left\langle-I_{4}\right\rangle$. Let $W$ be the natural module for $\mathrm{SU}(4, q)$. Take a hyperbolic basis $\left(u_{1}, v_{1}, u_{2}, v_{2}\right)$ for $W$ and define a basis $\left(e_{1}, f_{1}, e_{2}, f_{2}, w_{1}, w_{2}\right)$ for $W \wedge W$ thus:

$$
\begin{gathered}
e_{1}=u_{1} \wedge v_{2}, f_{1}=v_{1} \wedge u_{2}, e_{2}=-\psi\left(v_{1} \wedge v_{2}\right), f_{2}=\psi^{-1}\left(u_{1} \wedge u_{2}\right) \\
w_{1}=u_{1} \wedge v_{1}-u_{2} \wedge v_{2}, w_{2}=\psi\left(u_{1} \wedge v_{1}+u_{2} \wedge v_{2}\right) .
\end{gathered}
$$

The natural action of $\mathrm{SU}(4, q) /\left\langle-I_{4}\right\rangle$ on $W \wedge W$ coincides with the natural action of $\Omega^{-}(6, q)$, with the basis defined above, under the isomorphism defined below. The kernel of this homomorphism is, of course, $\left\langle-I_{4}\right\rangle$. Equating an element of $\mathrm{SU}(4, q)$ with its image modulo $\left\langle-I_{4}\right\rangle$, and using our notation for elements of $\mathrm{SU}(4, q)$, this homomorphism maps our chosen generators for $\mathrm{SU}(4, q)$ to $\Omega^{-}(6, q)$ as follows:

$$
\sigma \mapsto \tau^{-1}, \tau \mapsto \sigma, Z \mapsto U^{\prime}, \delta \mapsto \delta \delta^{-U^{\prime}}, \Delta \mapsto \delta, U \mapsto z U^{\prime 2}
$$


Theorem 11.1. If $q$ is odd, then a presentation for $\Omega^{-}(6, q)$ on our chosen generators is given by taking the presentation for $\mathrm{SU}(4, q) /\left\langle-I_{4}\right\rangle$ in Corollary 7.8 with the above substitutions.

11.1.3. A presentation for $\Omega^{-}(2 n, q)$ for $n \geq 4$. Let $N_{1}$ be the subgroup of $\Omega^{-}(2 n, q)$ generated by $\left\{z, U^{\prime}, V^{\prime}\right\}$. This is the same generating set as used in Theorem 10.1 for a subgroup of $\Omega(2 n-1, q)$, so the same presentation holds.

Let $N$ be the subgroup of $\Omega^{-}(2 n, q)$ generated by $\left\{\delta, z, U^{\prime}, V^{\prime}\right\}$. Now $\delta$ and its conjugates generate a subgroup of $N$ that is the direct product of the cyclic group $\langle\delta\rangle$ of order $\left(q^{2}-1\right) / 2$ with the direct product of $n-2$ cyclic groups of order $q-1$ generated by $\delta_{1} \delta_{2}^{-1}, \delta_{2} \delta_{3}^{-1}, \ldots, \delta_{n-2} \delta_{n-1}^{-1}$. This subgroup contains $U^{\prime 2}=\left(\delta_{1} \delta_{2}^{-1}\right)^{(q-1) / 2}$. Thus $N$ has order $(q+1)(q-1)^{n-1} 2^{n-2}(n-1)$ !, where a factor of $2^{n-1}$ arises from the $z_{i}$.

Theorem 11.2. Let $n \geq 4$. Let $G$ be the group generated by $\left\{\delta, z, U^{\prime}, V^{\prime}\right\}$ subject to the relations given below. Then $G$ is isomorphic to $N$.

(i) Defining relations for $N_{1}=\left\langle z, U^{\prime}, V^{\prime}\right\rangle$ as in Theorem 10.1.

(ii) $\left[\delta, U^{\prime V^{\prime}}\right]=1$.

(iii) If $n>4$ then $\left[\delta, V^{\prime} U^{\prime-1}\right]=1$.

(iv) $U^{\prime 2}=\left(\delta \delta^{-U^{\prime}}\right)^{(q-1) / 2}$.

(v) $\left[\delta, z^{U^{\prime}}\right]=\delta^{q-1}$.

(vi) $\delta^{\left(q^{2}-1\right) / 2}=1$.

(vii) $\left[\delta, \delta^{U^{\prime}}\right]=1$.

(viii) $\delta^{z}=\delta^{-1}$.

(ix) $\left[\delta^{q-1}, U^{\prime}\right]=1$.

Proof. By (i) we may take $G$ to contain $N_{1}$. By (iv) and (vii) it follows that $U^{\prime 2}$ commutes with $\delta$, so, using (ii) and (iii), we may assume that $\delta_{i} \in G$ for all $i$, and that $\left\langle U^{\prime}, V^{\prime}\right\rangle$ permutes these elements as unsigned permutations of the suffices. By (vii) it then follows that the $\delta_{i}$ all commute. The actions of $z_{1}$ and of $z_{2}$ on $\delta_{1}$ are given by (v) and (viii), and this gives the action of $z_{i}$ on $\delta_{j}$ for all $i$ and $j$. If $n>4$ it follows from (iii) and (ix), or from (ii) and (ix) if $n=4$, that $\left[\delta^{q-1}, V^{\prime}\right]=1$. Hence $\delta_{i}^{q-1}=\delta_{j}^{q-1}$ for all $i$ and $j$. Thus the order of the normal subgroup of $G$ generated by the $\delta_{i}$ has order dividing $(q-1)^{n-1}(q+1) / 2$. But $N_{1}$ has order $2^{2 n-3}(n-1)$ !, and contains a normal subgroup of order $2^{n-2}$, namely the subgroup generated by $U^{\prime 2}$ and its conjugates, that is contained in the subgroup generated by the $\delta_{i}$. So $G$ has order at most $(q+1)(q-1)^{n-1} 2^{n-2}(n-1)$ !, and as this is the order of $N$ the result follows.

Theorem 11.3. The centraliser of $\sigma=\sigma_{12}(1)$ in $N$ has index $2(n-1)(n-2)(q-1)$ in $N$ and is generated by the following elements:

(i) $U^{\prime V^{\prime 2}}$ if $n>4$;

(ii) $V^{\prime} U^{\prime-1} U^{\prime-V^{\prime}}$ if $n>5$;

(iii) $z^{V^{\prime 2}}$;

(iv) $\delta^{V^{\prime 2}}$;

(v) $\delta \delta^{U^{\prime}}$

(vi) $z z^{U^{\prime}} U^{\prime}$. 
Proof. The first claim follows from the fact that $N$ permutes transitively the $4(n-1)(n-$ $2)(q-1)$ elements $\sigma_{i j}(s)$, for $1 \leq|i| \neq|j| \leq n-1$ and $s \in \operatorname{GF}(q)^{\times}$, which are equal in pairs. The listed elements all centralise $\sigma$, and generate a group $G$ that contains a normal subgroup $K$ of order $(q+1) / 2$ generated by $\delta^{q-1}$. Observe that $G / K$ is isomorphic to the direct product of a copy of $D_{2(q-1)}<S_{n-3}$ generated by the images of the elements (i) to (iv) with a copy of $Q_{2(q-1)}$ generated by the images of the elements (v) and (vi). So $G$ has order $(q+1)(q-1)^{n-2} 2^{n-3}(n-3)$ !, which is the order of the centraliser.

Since $\tau_{i}(\alpha)^{\delta_{i}}=\tau_{i}\left(\alpha \omega^{2}\right)$ for $i>0$, and $\tau_{i}(\alpha)^{\delta_{i}}=\tau_{i}\left(\alpha \omega^{-2}\right)$ for $i<0$, it follows that $\left\{\tau_{ \pm i}(\alpha) \mid 1 \leq i \leq n-1, \alpha \in \mathrm{GF}\left(q^{2}\right)^{\times}\right\}$is permuted under conjugation by $N$, and falls into two orbits, the elements for which $\alpha$ is a square forming one orbit, and those for which $\alpha$ is a non-square forming the other.

Theorem 11.4. The centraliser of $\tau=\tau_{1}(1)$ in $N$ has index $(n-1)\left(q^{2}-1\right)$ in $N$ and is generated by the following elements:

(i) $\delta^{(q+1) / 2} \delta^{U}$;

(ii) $U^{\prime} z U^{\prime}$;

(iii) $U^{\prime V^{\prime}}$;

(iv) $V^{\prime} U^{\prime-1}$ if $n>4$.

Proof. These elements all centralise $\tau$. The first two generators, which as matrices are $\operatorname{diag}\left(-1,-1, \omega_{0}^{-1}, \omega_{0}, 1,1, \ldots, 1,-1,-1\right)$ and $\left(e_{1}\right)^{-}\left(f_{1}\right)^{-}\left(e_{2},-f_{2}\right)\left(w_{1}\right)^{-}$, lie in $\Omega^{-}(6, q)$; they generate a copy of $D_{2(q-1)}$. So these elements, together with items (iii) and (iv), generate a copy of $D_{2(q-1)} 2 S_{n-2}$, which has the required order $(2(q-1))^{n-2}(n-2)$ !.

Theorem 11.5. Let $q=p^{e}$ for an odd prime $p$, and let $n \geq 4$. Let $G$ be the group generated by $\left\{\sigma, \tau, \delta, z, U^{\prime}, V^{\prime}\right\}$ subject to the relations given below. Then $G$ is isomorphic to $\Omega^{-}(2 n, q)$.

(i) Defining relations for $N=\left\langle\delta, z, U^{\prime}, V^{\prime}\right\rangle$ as in Theorem 11.2, but omitting relations (iv) to (ix) of that theorem, relations (iv), (v) and (vi) of Theorem 10.1, and the relation $U^{\prime 4}=1$ from the presentation for $\left\langle U^{\prime}, V^{\prime}\right\rangle$.

(ii) Relations that state that the elements listed in Theorem 11.3 centralise $\sigma$, omitting relations ( $v$ ) and (vi).

(iii) Relations that state that the elements listed in Theorem 11.4 centralise $\tau$, omitting relations (i) and (ii).

(iv) Relations that present $\Omega^{-}(6, q)$ on $\left\{\sigma, \tau, \delta, z, U^{\prime}\right\}$ as in Theorem 11.1 .

(v) The Steinberg relations $(i v)(a)$ to $(i v)(c)$, and $(i v)(d)$ when $n>4$, of Theorem 9.4 .

(vi) The Steinberg relation $\left[\sigma^{V^{\prime}}, \tau\right]=1$.

Proof. The relations omitted from (i), (ii), and (iii) are supplied by (iv).

The relations (iv)(e) and (iv)(f) of Theorem 9.4 are supplied respectively by (iv) of this theorem, and by (iv)(d) of Theorem 9.4 on conjugation by $z_{l}$.

All Steinberg relations involving $\sigma_{i j}(s)$ and $\tau_{k}(\alpha)$, when at most two values for the moduli of the suffices $i, j, k$ arise, may be deduced from (i) to (iv).

We now consider Steinberg relations whose LHS is of the form $\left[\sigma_{i j}(s), \sigma_{k l}(t)\right]$, where the moduli of the suffices take at least three distinct values. Let $H$ denote the subgroup of $\Omega^{-}(2 n, q)$ generated by $\left\{\sigma, \delta, z, U^{\prime}, V^{\prime}\right\}$. Observe that $H$ contains $N$ and fixes 
$\left\langle e_{1}, f_{1}, \ldots, e_{n-1}, f_{n-1}\right\rangle$, acting on this space as $\mathrm{O}^{+}(2(n-1), q)$. Let $N_{0}$ denote the torus normaliser in $\Omega^{+}(2(n-1), q)$ denoted by $N$ in Section 9 . So $N$ contains $N_{0}$ as a subgroup of index 2(q+1), and the Steinberg relations (iv) of Theorem 9.4 suffice a fortiori to imply all the Steinberg relations of the type under consideration.

It remains to consider the Steinberg relations $\left[\sigma_{i j}(s), \tau_{k}(\alpha)\right]=1$, where the suffices have distinct moduli. These follow from the one instance $\left[\sigma_{23}(1), \tau_{1}(1)\right]=1$ given by $(\mathrm{v})$.

The standard argument now shows that $G$ is generated by the Steinberg generators.

11.1.4. A presentation for $\mathrm{P} \Omega^{-}(2 n, q)$. The centre of $\Omega^{-}(2 n, q)$ is trivial, unless $q^{n} \equiv$ $3 \bmod 4$, when it has order 2 . If it is non-trivial, then it is generated by $V^{\prime(n-1)} \delta^{\left(q^{2}-1\right) / 4}$. For $V^{\prime(n-1)}=\operatorname{diag}(-1,-1, \ldots,-1,1,1)$ as $n$ is odd, and $\delta^{\left(q^{2}-1\right) / 4}=\operatorname{diag}(1,1, \ldots, 1,-1,-1)$ as $q \equiv 3 \bmod 4$.

11.1.5. Standard generators for $\Omega^{-}(2 n, q)$. In Table 2 of [21] the standard generators for $\Omega^{-}(2 n, q)$ are labelled $s, t, \delta, u, v$. Observe that $s=z^{V^{\prime-1}} ; \delta=\left(\delta^{V^{\prime-1}}\right)^{\left((q-1)^{2} / 2\right)-1} ; u=U^{\prime}$; $v=V^{\prime}$; if $d \equiv 0 \bmod 4$ then $t=\tau^{V^{\prime-1}}$, else $t=\left(\tau^{-1}\right)^{V^{\prime-1}}$. The presentation generators $\sigma=\left[\tau, \tau^{z U^{\prime}}\right]^{(p-1) / 2}$ and $\delta=\left(\delta^{v}\right)^{\left((q-1)^{2} / 2\right)-1}$.

\subsection{Even characteristic.}

11.2.1. Generators and notation. Let $q=2^{e}$. Let $\omega$ be a primitive element of $\operatorname{GF}\left(q^{2}\right)$, and let $\omega_{0}=\omega^{q+1}$, so $\omega_{0}$ is a primitive element of $\operatorname{GF}(q)$.

Let $n>2$. We take a basis $\left(e_{1}, f_{1}, e_{2}, f_{2}, \ldots, e_{n-1}, f_{n-1}, w_{1}, w_{2}\right)$ for the natural module, where the associated quadratic form has matrix

$$
\operatorname{diag}\left(\left(\begin{array}{ll}
0 & 1 \\
0 & 0
\end{array}\right), \ldots,\left(\begin{array}{ll}
0 & 1 \\
0 & 0
\end{array}\right),\left(\begin{array}{cc}
1 & \omega+\omega^{q} \\
0 & \omega_{0}
\end{array}\right)\right)
$$

and the corresponding bilinear form has matrix

$$
\operatorname{diag}\left(\left(\begin{array}{ll}
0 & 1 \\
1 & 0
\end{array}\right), \ldots,\left(\begin{array}{ll}
0 & 1 \\
1 & 0
\end{array}\right),\left(\begin{array}{cc}
0 & \omega+\omega^{q} \\
\omega+\omega^{q} & 0
\end{array}\right)\right) \text {. }
$$

This form is of type - .

We define the following elements of $\Omega^{-}(2 n, q)$, where $1 \leq i, j \leq n-1$ and $i \neq j$, and $s \in \mathrm{GF}(q)$, and $\alpha \in \mathrm{GF}\left(q^{2}\right)$.

$$
\begin{aligned}
& \delta_{i}=\left(e_{i} \mapsto \omega_{0}^{-1} e_{i}, f_{i} \mapsto \omega_{0} f_{i}, w_{1} \mapsto A w_{1}+C w_{2}, w_{2} \mapsto B w_{1}+w_{2}\right), \text { where } \\
& A=\omega^{q-1}+\omega^{1-q}+1 \\
& B=\omega+\omega^{q} \\
& C=\omega^{-1}+\omega^{-q} ; \\
& z_{i}=\left(e_{i} \mapsto f_{i}, f_{i} \mapsto e_{i}, w_{2} \mapsto w_{2}+B w_{1}\right) ; \\
& \sigma_{i j}(s)=\left(e_{i} \mapsto e_{i}+s e_{j}, f_{j} \mapsto f_{j}+s f_{i}\right) ; \\
& \sigma_{-i, j}(s)=\left(f_{i} \mapsto f_{i}+s e_{j}, f_{j} \mapsto f_{j}+s e_{i}\right)=\sigma_{i j}(s)^{z_{i}} ; \\
& \sigma_{i,-j}(s)=\left(e_{i} \mapsto e_{i}+s f_{j}, e_{j} \mapsto e_{j}+s f_{i}\right)=\sigma_{i j}(s)^{z_{j}} ; \\
& \sigma_{-i,-j}(s)=\sigma_{j i}(s)=\sigma_{i j}(s)^{z_{i} z_{j}} ; \\
& \tau_{i}(\alpha)=\left(e_{i} \mapsto e_{i}+\alpha^{q+1} f_{i}+\left(\alpha^{q} \omega^{q}+\alpha \omega\right) B^{-1} w_{1}+\left(\alpha^{q}+\alpha\right) B^{-1} w_{2},\right. \\
& \left.w_{1} \mapsto w_{1}+\left(\alpha+\alpha^{q}\right) f_{i}, w_{2} \mapsto w_{2}+\left(\omega \alpha+\omega^{q} \alpha^{q}\right) f_{i}\right) ; \\
& \tau_{-i}(\alpha)=\left(f_{i} \mapsto f_{i}+\alpha^{q+1} e_{i}+\left(\alpha^{q} \omega+\alpha \omega^{q}\right) B^{-1} w_{1}+\left(\alpha^{q}+\alpha\right) B^{-1} w_{2},\right. \\
& \left.w_{1} \mapsto w_{1}+\left(\alpha+\alpha^{q}\right) e_{i}, w_{2} \mapsto w_{2}+\left(\alpha^{q} \omega+\alpha \omega^{q}\right) e_{i}\right)=\tau_{i}(\alpha)^{z_{i}} ;
\end{aligned}
$$


$U=\left(e_{1}, e_{2}\right)\left(f_{1}, f_{2}\right)$

$V=\left(e_{1}, e_{2}, \ldots, e_{n-1}\right)\left(f_{1}, f_{2}, \ldots, f_{n-1}\right)$.

All these elements of $\Omega^{-}(2 n, q)$ centralise those basis elements that they are not stated to move. Observe that $\delta_{i}^{z_{i}}=\delta_{i}^{-1}$.

We give a presentation for $\Omega^{-}(2 n, q)$ on the generating set $\left\{\sigma=\sigma_{12}(1), \tau=\tau_{1}(1), \delta=\right.$ $\left.\delta_{1}, z=z_{1}, U, V\right\}$, omitting $V$ if $n=3$.

11.2.2. A presentation for $\Omega^{-}(6, q)$. We exploit the isomorphism between $\Omega^{-}(6, q)$ and $\mathrm{SU}(4, q)$. The exterior square of the natural $\mathrm{SU}(4, q)$-module $W$ may be written over $\mathrm{GF}(q)$, giving rise to the natural representation of $\Omega^{-}(6, q)$, as follows. Take a hyperbolic basis $\left(u_{1}, v_{1}, u_{2}, v_{2}\right)$ for $W$, and define a basis $\left(e_{1}, f_{1}, e_{2}, f_{2}, w_{1}, w_{2}\right)$ for $W \wedge W$ thus:

$$
\begin{aligned}
& e_{1}=u_{1} \wedge v_{2}, f_{1}=v_{1} \wedge u_{2}, e_{2}=v_{1} \wedge v_{2}, f_{2}=u_{1} \wedge u_{2} \\
& w_{1}=u_{1} \wedge v_{1}+u_{2} \wedge v_{2}, w_{2}=\omega u_{1} \wedge v_{1}+\omega^{q} u_{2} \wedge v_{2} .
\end{aligned}
$$

The resulting isomorphism maps our chosen generators for $\mathrm{SU}(4, q)$ to $\Omega^{-}(6, q)$ as follows: $\sigma \mapsto \tau, \tau \mapsto \sigma, Z \mapsto U, \delta \mapsto \delta \delta^{-U}, \Delta \mapsto \delta, U \mapsto z$. Theorem 7.7 now provides the desired presentation for $\Omega^{-}(6, q)$.

11.2.3. A presentation for $\Omega^{-}(2 n, q)$ for $n \geq 4$. Let $N_{1}$ be the subgroup of $\Omega^{-}(2 n, q)$ generated by $\{z, U, V\}$. This is a copy of $C_{2} \prec S_{n-1}$.

Theorem 11.6. Let $n \geq 4$. Let $G$ be the group generated by $\{z, U, V\}$ subject to the relations given below. Then $G$ is isomorphic to $N_{1}$.

(i) Defining relations for $S_{n-1}$ on $\{U, V\}$.

(ii) $\left[z, U^{V}\right]=1$.

(iii) If $n>4$ then $[z, V U]=1$.

(iv) $z^{2}=1$.

(v) $\left[z, z^{U}\right]=1$.

Proof. This is a standard wreath product presentation.

Let $N$ be the subgroup of $\Omega^{-}(2 n, q)$ generated by $\{\delta, z, U, V\}$. Now $\delta$ and its conjugates generate a subgroup of $N$ that is the direct product of the cyclic group $\langle\delta\rangle$ of order $q^{2}-1$ with the direct product of $n-2$ cyclic groups of order $q-1$ generated by $\delta_{1} \delta_{2}^{-1}, \delta_{2} \delta_{3}^{-1}, \ldots, \delta_{n-2} \delta_{n-1}^{-1}$. Thus $N$ has order $(q+1)(q-1)^{n-1} 2^{n-1}(n-1)$ !, twice that for odd $q$, reflecting the fact that $\delta$ has order $q^{2}-1$ if $q$ is even, and order $\left(q^{2}-1\right) / 2$ if $q$ is odd.

Theorem 11.7. Let $n \geq 4$. Let $G$ be the group generated by $\{\delta, z, U, V\}$ subject to the relations given below. Then $G$ is isomorphic to $N$.

(i) Defining relations for $N_{1}=\langle z, U, V\rangle$ as in Theorem 11.6.

(ii) $\left[\delta, U^{V}\right]=1$.

(iii) If $n>4$ then $[\delta, V U]=1$.

(iv) $\left[\delta, z^{U}\right]=\delta^{q-1}$.

(v) $\delta^{q^{2}-1}=1$.

(vi) $\left[\delta, \delta^{U}\right]=1$.

(vii) $\delta^{z}=\delta^{-1}$. 
(viii) $\left[\delta^{q-1}, U\right]=1$.

The proof is essentially identical to that of Theorem 11.2 ,

Theorem 11.8. The centraliser of $\sigma=\sigma_{12}(1)$ in $N$ has index $2(n-1)(n-2)(q-1)$ in $N$ and is generated by the following elements:

(i) $U^{V^{2}}$ if $n>4$;

(ii) $V U U^{V}$ if $n>5$;

(iii) $z^{V^{2}}$;

(iv) $\delta^{V^{2}}$;

(v) $\delta \delta^{U}$;

(vi) $z z^{U} U$.

The proof is essentially identical to that of Theorem 11.3 .

As in the case of odd characteristic, $\tau_{i}(\alpha)^{\delta_{i}}=\tau_{i}\left(\alpha \omega^{2}\right)$ for $i>0$, and $\tau_{i}(\alpha)^{\delta_{i}}=\tau_{i}\left(\alpha \omega^{-2}\right)$ for $i<0$. Now $N$ acts transitively by conjugation on $\left\{\tau_{ \pm i}(\alpha) \mid 1 \leq i \leq n-1, \alpha \in \operatorname{GF}\left(q^{2}\right)^{\times}\right\}$, a set of size $2\left(q^{2}-1\right)(n-1)$.

Theorem 11.9. The centraliser of $\tau=\tau_{1}(1)$ in $N$ has index $2\left(q^{2}-1\right)(n-1)$ in $N$ and is generated by the following elements:

(i) $\delta^{V^{2}} \delta^{-V}$;

(ii) $z^{U}$;

(iii) $U^{V}$;

(iv) $V U$ if $n>4$.

Proof. The centraliser of $\tau$ in $N$ is generated by the subgroup of $\langle U, V\rangle$ that as permutations of $\{1,2, \ldots, n-1\}$ fixes 1 , together with $\left\{z_{i} \mid i>1\right\}$ and $\left\{\delta_{i} \delta_{2}^{-1} \mid i>2\right\}$ and $\delta_{1}^{q-1} \delta_{2}^{2}$, and hence is generated by $\left\{\delta^{V^{2}} \delta^{-V}, z^{U}, U^{V}, V U, \delta^{q-1} \delta^{2 U}\right\}$, omitting $V U$ if $n=4$. This group has index $2\left(q^{2}-1\right)(n-1)$ in $N$, as required, the final generator having order $q-1$. But $\delta^{q-1} \delta^{2 U}=\left[\delta^{V^{2}} \delta^{-V}, z^{U}\right]$, so this generator is redundant.

Theorem 11.10. Let $q$ be even and let $n \geq 4$. Let $G$ be the group generated by $\{\sigma, \tau, \delta, z, U, V\}$ subject to the relations given below. Then $G$ is isomorphic to $\Omega^{-}(2 n, q)$.

(i) Defining relations for $N=\langle\delta, z, U, V\rangle$ as in Theorem 11.7, but omitting relations (iv) and (v) from Theorem 11.6, relations (iv) to (viii) of Theorem 11.7, and the relation $U^{2}=1$ from the presentation for $\langle U, V\rangle$.

(ii) Relations that state that the elements listed in Theorem 11.8 centralise $\sigma$, omitting relations (v) and (vi).

(iii) Relations that state that the elements listed in Theorem 11.9 centralise $\tau$, but omitting the relation $\left[\tau, z^{U}\right]=1$.

(iv) Relations that present $\Omega^{-}(6, q)$ on $\{\sigma, \tau, \delta, z, U\}$ as in Theorem 7.7 .

(v) The Steinberg relations (iv)(a) to (iv)(c), and (iv)(d) when $n>4$, of Theorem 9.9.

(vi) The Steinberg relation $\left[\sigma^{V}, \tau\right]=1$.

Proof. The proof of Theorem 11.5 carries over with no significant change.

If $q$ is even, then $\Omega^{-}(2 n, q)$ is simple. 
11.2.4. Standard generators for $\Omega^{-}(2 n, q)$. In Table 1 of [10] the non-trivial standard generators for $\Omega^{-}(2 n, q)$ are labelled $s, t, \delta, u, v$. Observe that $s=z^{V^{-1}} ; u=U ; v=V$; the

standard generator $\delta$ is $\left(\delta^{-1}\right)^{V^{-1}}$; if $d \equiv 0 \bmod 4$ then $t=\tau^{V^{-1}}$, else $t=\left(\tau^{-1}\right)^{V^{-1}}$. As a word in the remaining presentation generators, $\sigma=\left[\tau^{\delta^{V}}, \tau^{U}\right]^{z U\left(\delta^{-V}\right)^{(q-m)}}$, where $m$ is defined by $\omega_{0}^{m}=\omega^{2}+\omega^{2 q}$, so $\sigma$ can be written as an explicit word in the standard generators. (This arises from relation (v)(b) of Theorem 7.7.)

\section{VERIFICATION AND ACCESS TO RESULtS}

Both the presentations and definitions of their (presentation and standard) generators are publicly available [22] in MAGMA. We store the relators as straight-line programs on the generating set.

We have run extensive checks to verify their correctness. By evaluating the relations, it is easy to demonstrate that a classical group of specified dimension $d$ and field size $q$ is a quotient of the corresponding finitely-presented group.

For each type, we have a presentation for $N$, the normaliser of a maximal torus in a split $B N$ pair. For an extensive range of $d$ and $q$, typically where the corresponding $N$ has order at most $10^{9}$, we verified using coset enumeration that this presentation defines $N$. We constructed the permutation action of $N$ on unordered pairs of root elements, confirming that the orbits and representatives are as claimed. Extensive checks were also performed in the classical group: in particular, the claimed (generating sets and structure of) centralisers in $N$ of root elements have been checked. For relevant $d \leq 10$ and $q \leq 9$ (and sometimes for larger $d$ and smaller $q$ ), we also carried out coset enumeration in each finitely-presented group over the preimage of the maximal subgroup of smallest index, and verified that the resulting permutation representation defines (the central quotient of) the claimed classical group.

We observe that our presentations seem particularly well-suited to coset enumeration, often defining precisely the required number of cosets with no redundancy.

\section{REFERENCES}

[1] H. Bäärnhielm, D.F. Holt, C.R. Leedham-Green, and E.A. O'Brien. A practical model for computation with matrix groups. J. Symbolic Comput., 68, 27-60, 2015.

[2] W. Bosma, J. Cannon, and C. Playoust. The Magma algebra system I: The user language, J. Symbolic Comput., 24, 235-265, 1997.

[3] László Babai and Endre Szemerédi. On the complexity of matrix group problems, I. In Proc. 25th IEEE Sympos. Foundations Comp. Sci., pages 229-240, 1984.

[4] L. Babai, A.J. Goodman, W.M. Kantor, E.M. Luks, and P.P. Pálfy. Short presentations for finite groups. J. Algebra, 194, 79-112, 1997.

[5] John Bray, M.D.E. Conder, C.R. Leedham-Green, and E.A. O'Brien. Short presentations for alternating and symmetric groups. Trans. Amer. Math. Soc., 363, 3277-3285, 2011.

[6] C.M. Campbell and E.F. Robertson. A deficiency zero presentation for SL(2,p). Bull. London Math. Soc., 12, 17-20, 1980.

[7] C.M. Campbell, E.F. Robertson, and P.D. Williams. On Presentations of PSL(2, $\left.p^{n}\right)$. J. Austral. Math. Soc (Series A), 48, 333-346, 1990.

[8] Arjeh M. Cohen, Scott H. Murray, and D. E. Taylor. Computing in groups of Lie type. Math. Comp., 73, 1477-1498, 2004. 
[9] Elliot Costi. Constructive membership testing in classical groups. PhD thesis, Queen Mary, University of London, 2009.

[10] Heiko Dietrich, C.R. Leedham-Green, Frank Lübeck, and E.A. O'Brien. Constructive recognition of classical groups in even characteristic, J. Algebra, 391, 227-255, 2013.

[11] Heiko Dietrich, C.R. Leedham-Green and E.A. O'Brien. Effective black-box constructive recognition of classical groups, J. Algebra, 421, 460-492, 2015.

[12] Daniel Gorenstein, Richard Lyons, and Ronald Solomon. The classification of the finite simple groups. Number 3. Part I. Chapter A. Almost simple K-groups. Mathematical Surveys and Monographs, 40.3. American Mathematical Society, Providence, RI, 1998. xvi+419 pp.

[13] R.M. Guralnick, W.M. Kantor, M. Kassabov and A. Lubotzky. Presentations of finite simple groups: a quantitative approach. J. Amer. Math. Soc., 21, 711-774, 2008.

[14] R.M. Guralnick, W.M. Kantor, M. Kassabov, and A. Lubotzky. Presentations of finite simple groups: a computational approach. J. Eur. Math. Soc., 13, 391-458, 2011.

[15] R.B. Howlett, L.J. Rylands and D.E. Taylor. Matrix generators for exceptional groups of Lie type, J. Symbolic Comput., 31, 429-445, 2001.

[16] Alexander Hulpke and Ákos Seress. Short presentations for three-dimensional unitary groups. J. Algebra, 245, 719-729, 2001.

[17] Peter Huxford. Short presentations of finite simple groups. https://arxiv.org/abs/1907.10828, 2019.

[18] Sebastian Jambor, Martin Leuner, Alice C. Niemeyer, and Wilhelm Plesken. Fast recognition of alternating groups of unknown degree. J. Algebra, 392, 315-335, 2013.

[19] D.L. Johnson. Presentations of Groups, volume 15 of London Math. Soc. Stud. Texts. Cambridge University Press, Cambridge, 1990.

[20] W. M. Kantor and Á. Seress. Black box classical groups. Mem. Amer. Math. Soc. 149, 2001.

[21] C.R. Leedham-Green and E.A. O'Brien. Constructive recognition of classical groups in odd characteristic, J. Algebra, 322, 833-881, 2009.

[22] C.R. Leedham-Green and E.A. O’Brien. www.math.auckland.ac.nz/〜 obrien/Presentations.tar, 2019.

[23] Martin W. Liebeck and E.A. O'Brien. Recognition of finite exceptional groups of Lie type. Trans. Amer. Math. Soc., 368, 6189-6226, 2016.

[24] E.H. Moore. Concerning the abstract groups of order $k$ ! and $\frac{1}{2} k$ ! holohedrically isomorphic with the symmetric and the alternating substitution groups on $k$ letters. Proc. London Math. Soc., 28, 357-366 (1897).

[25] E.A. O'Brien. Algorithms for matrix groups. Groups St Andrews 2009 in Bath II, London Math. Soc. Lecture Note Series 388, 297-323, 2011.

[26] Ákos Seress. Permutation Group Algorithms. Cambridge Tracts in Mathematics 152. Cambridge University Press, 2003.

[27] Robert Steinberg. Générateurs, relations et revêtements de groupes algébriques. In Colloq. Théorie des Groupes Algébriques (Bruxelles, 1962), pp. 113-127. Librairie Universitaire, Louvain, 1962.

[28] Robert Steinberg. Lectures on Chevalley groups. Notes prepared by John Faulkner and Robert Wilson. Yale University, New Haven, Conn., 1968. iii+277 pp.

[29] Robert Steinberg. Generators, relations and coverings of algebraic groups. II. J. Algebra, 71, 527-543, 1981.

[30] Michio Suzuki. On a class of doubly transitive groups. Ann. of Math. (2), 75, 105-145, 1962.

[31] Robert A. Wilson. Standard generators for sporadic simple groups. J. Algebra, 184(2):505-515, 1996.

[32] R.A. Wilson et al. Atlas of Finite Group Representations. brauer.maths.qmul.ac.uk/Atlas

School of Mathematical Sciences, Queen Mary, University of London, London E1 4NS, United Kingdom

E-mail address: c.r.leedham-green@qmul.ac.uk

Department of Mathematics, University of Auckland, Auckland, New Zealand

E-mail address: e.obrien@auckland.ac.nz 